\title{
Experimental Investigation of AGET ATRP Polymerization of Butyl Methacrylate in an Emulsion Stirred Tank Reactor
}

By

John Tam

\author{
A Thesis \\ Presented to Ryerson University \\ in partial fulfillment of the requirements for the degree of \\ Master of Applied Science \\ in the Program of Chemical Engineering
}

Toronto, Ontario, Canada, 2020.

CJohn Tam, 2020. 


\section{AUTHOR'S DECLARATION}

I hereby declare that I am the sole author of this thesis. This is a true copy of the thesis, including any required final revisions, recommended by the thesis examiners.

I authorize Ryerson University to lend this thesis to other institutions or individuals for the purpose of scholarly research.

I further authorize Ryerson University to reproduce this thesis by photocopying or by other means, in total or in part, at the request of other institutions or individuals for the purpose of scholarly research.

I understand that my thesis may be made electronically available to the public.

Author's Signature

John Tam 


\title{
ABSTRACT
}

\section{Experimental Investigation of AGET ATRP Polymerization of Butyl Methacrylate in an Emulsion Stirred Tank Reactor}

\author{
John Tam \\ Master of Applied Science, 2020 \\ Chemical Engineering Department \\ Ryerson University
}

This study investigates ATRP emulsion polymerization of butyl methacrylate (BMA) in a 2-L stirred tank reactor using AGET as the initiation technique with ascorbic acid. The polymerization is performed in two step procedure using surfactant (Brij 98) in distilled water. The reaction is initiated by the catalyst $\mathrm{CuBr} 2 / \mathrm{dNbpy}$ and initiator $\mathrm{EBiB}$ under a blanket of nitrogen to minimize air presence. An experimental design is performed to investigate the effects of the key variables: temperature, catalyst complex, surfactant and reducing agent. For reaction temperatures of $50^{\circ} \mathrm{C}$, $60^{\circ} \mathrm{C}$ and $70^{\circ} \mathrm{C}$, BMA conversion obtained is $63.9 \%, 70.2 \%$ and $85.8 \%$, respectively. All other nine tests are done at $70^{\circ} \mathrm{C}$ for appropriate amounts of reactants. The results concluded that BMA conversion improves to $90 \%$ and the PDI increases slightly from 1.15 to 1.29 for more ascorbic acid. BMA conversion and PDI improve with less surfactant, but more ligand narrows MWD and reduces the catalyst activity. 


\section{ACKNOWLEDGMENTS}

I would like to thank my supervisor Dr Ramdhane Dhib for his unwavering support, patience, financial support and guidance throughout my graduate experience. I would also like to express my gratitude to my co-supervisor, Dr. Mehrab Mehrvar, for his support and encouragement during my research. I would also like to express my gratitude to Dr Daniel Foucher and research team for guidance and sharing the analytical equipment. I extend my thanks to my thesis committee members: Professor Philip Chan and Professor Hadis Zarrin for their time and consideration.

I would also like to thank the assistance of the engineering staffs, Ali Hemmati, Daniel Boothe, and Tondar Tajrobekar, for all the support in troubleshooting the equipment and ensuring the arrival of materials on time.

To my colleagues throughout the years, with them, life was enjoyable in the lab and campus. Thank you for the help and advice. I learned a lot from them.

Finally, I am forever grateful to my family for their enduring support, patience and understanding in my pursuit for personal growth. 


\section{Table of Contents}

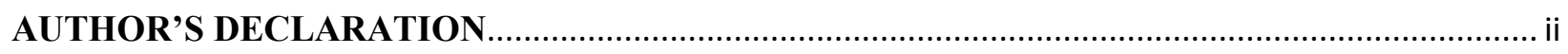

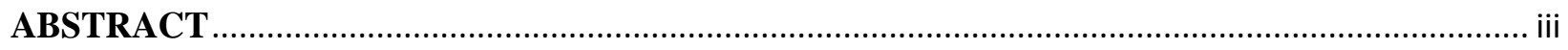

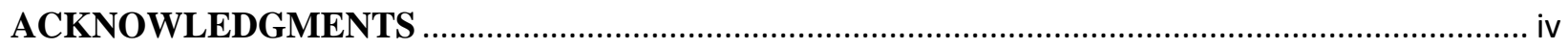

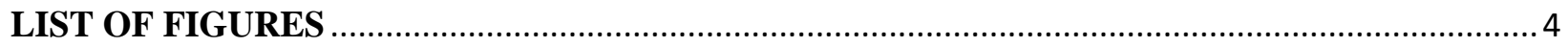

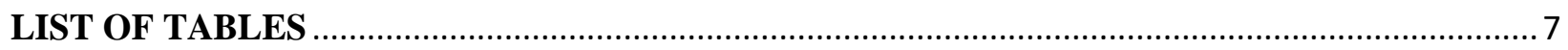

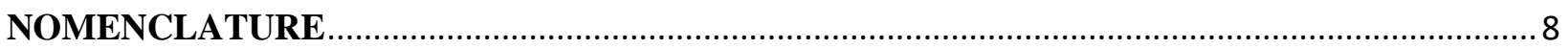

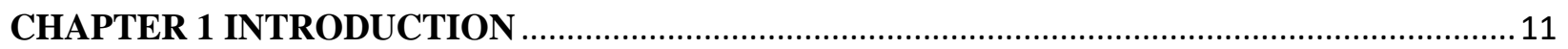

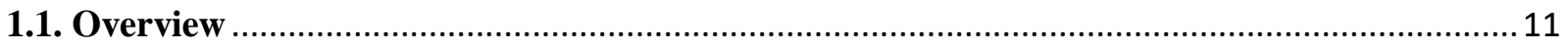

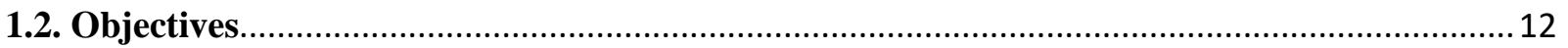

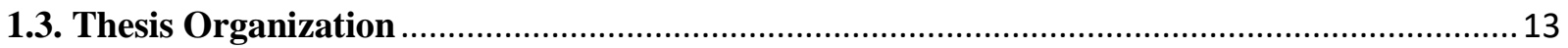

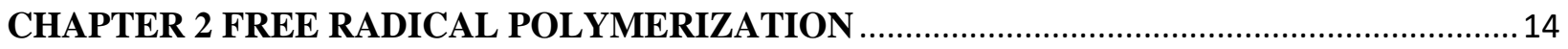

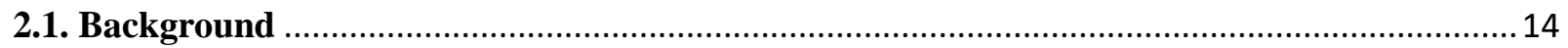

2.2. Conventional Radical Polymerization: Free Radical Polymerization...................................16

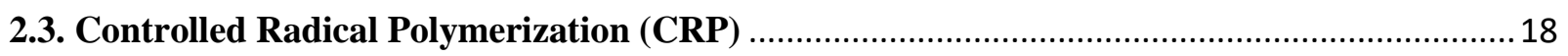

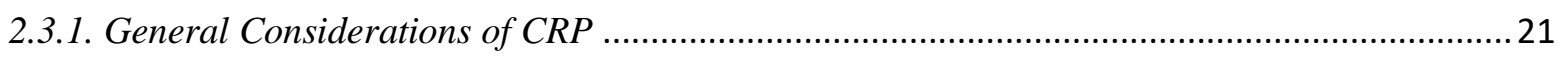

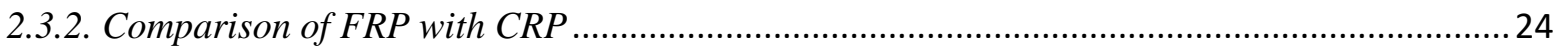

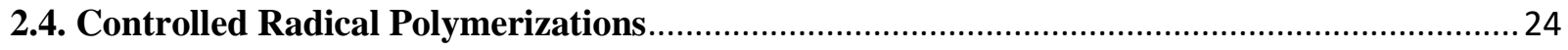

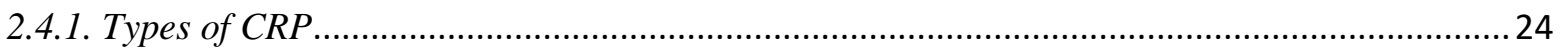

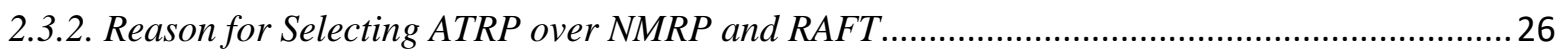

CHAPTER 3 ATOM TRANSFER RADICAL POLYMERIZATION ...........................................28

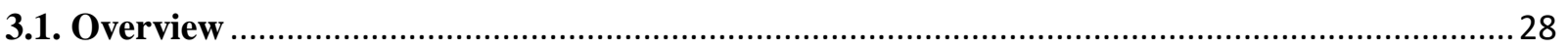

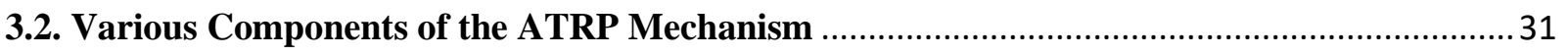

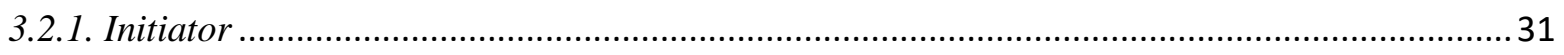

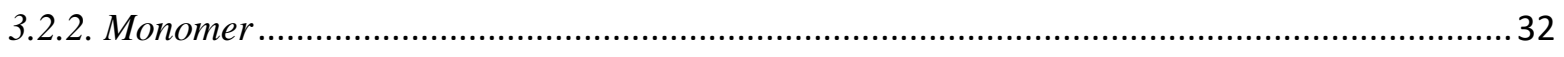

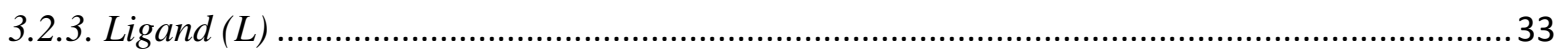

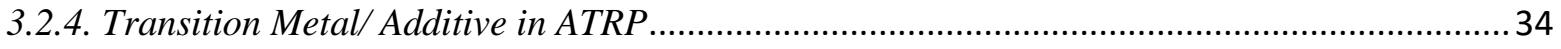

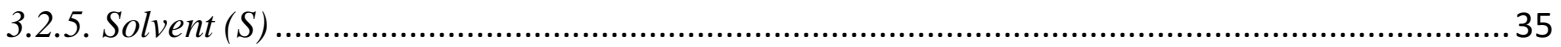




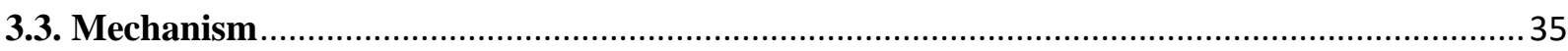

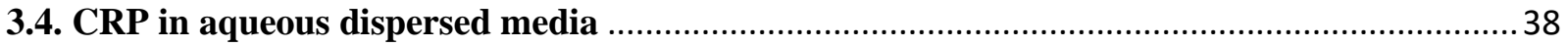

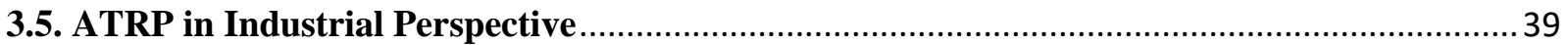

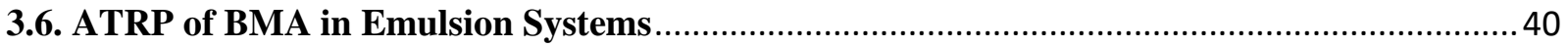

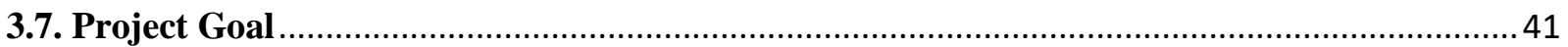

CHAPTER 4 EXPERIMENTAL PROCEDURE AND CHARACTERIZATION TECHNIQUES .42

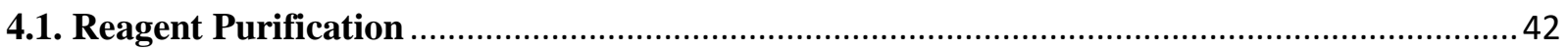

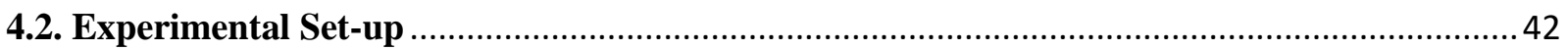

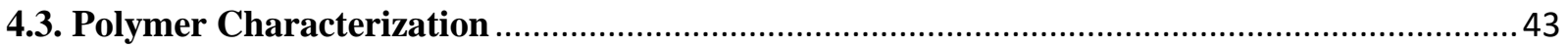

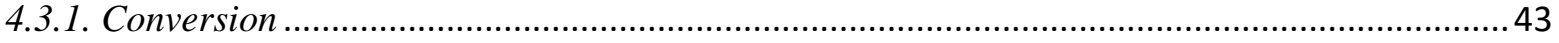

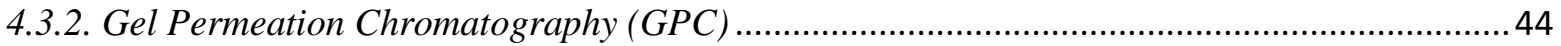

4.3.3. Quantification and Molar Mass Distribution ..................................................................... 45

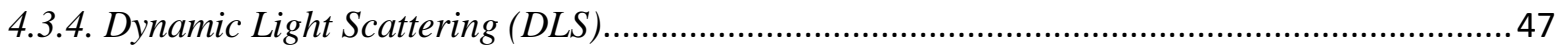

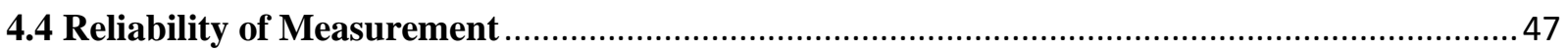

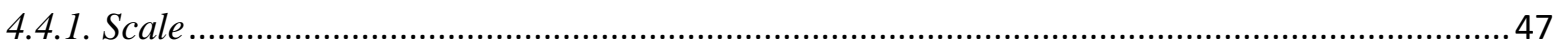

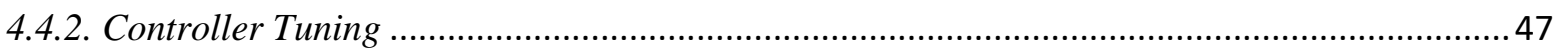

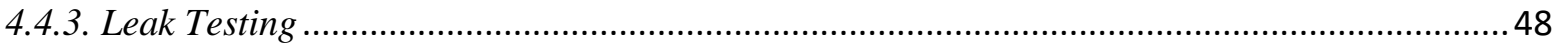

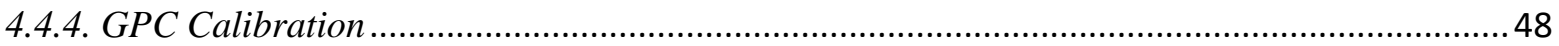

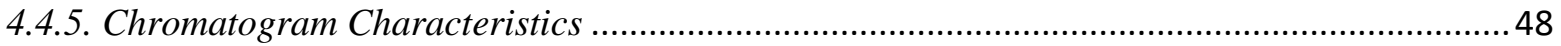

CHAPTER 5 PRELIMINARY INVESTIGATION OF AGET ATRP OF BMA IN EMULSION

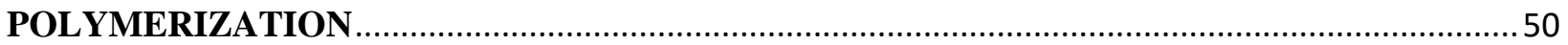

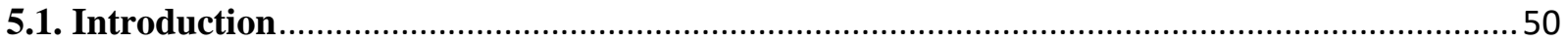

5.2. Description of Experimental Procedure …….................................................................... 51

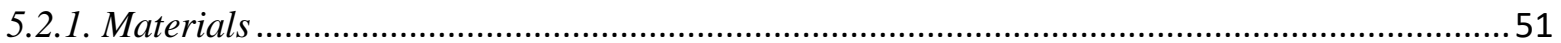

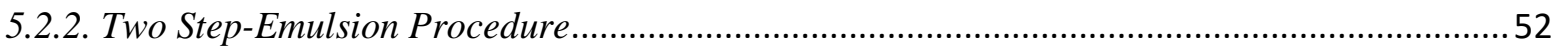

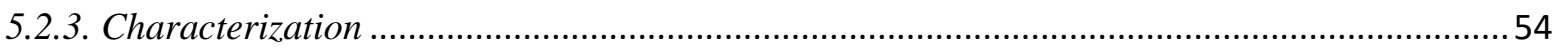

5.3. Preliminary Investigation of Two-Step Emulsion Procedure …........................................... 54

5.3.1. Ligand Selection........................................................................................................... 54

5.3.2. Establishment of two-step polymerization method ............................................................. 57

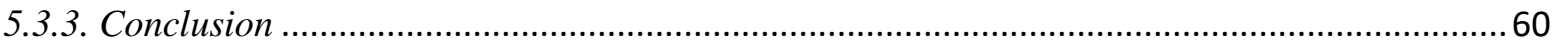

CHAPTER 6 INVESTIGATION OF AGET ATRP OF BMA IN EMULSION

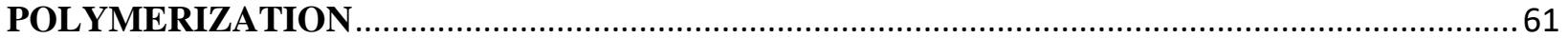


6.1. Temperature

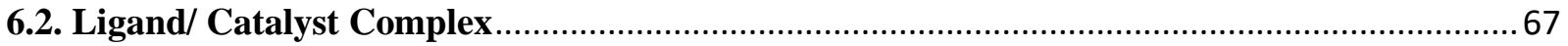

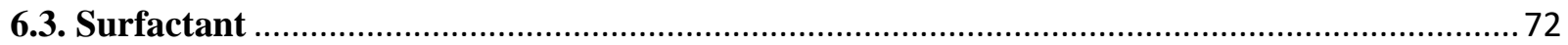

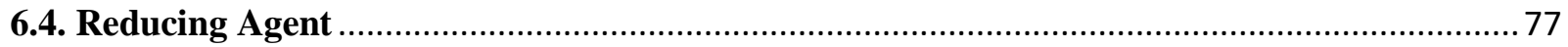

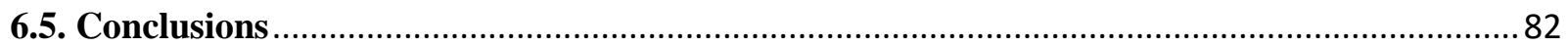

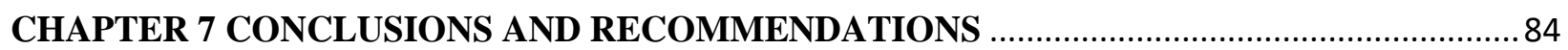

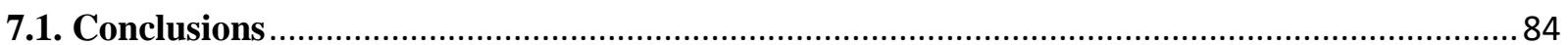

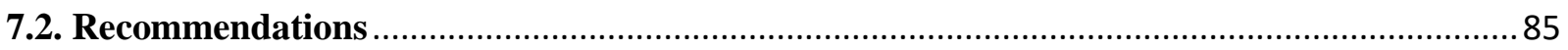

APPENDIX A :

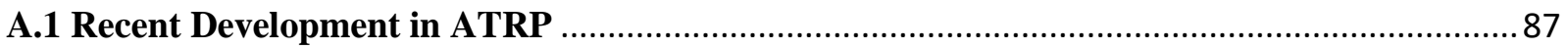

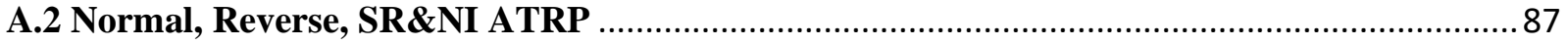

A.3 Activator Generated by Electron Transfer (AGET) ATRP ............................................. 88

A.4 Initiators for Continuous Activator Regeneration (ICAR) ATRP ..................................... 88

A.5 Activator Regenerated by Electron Transfer (ARGET) ATRP ….................................... 89

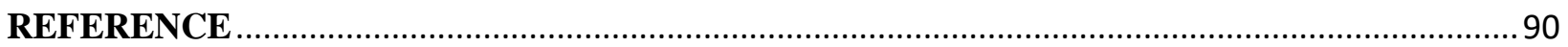




\section{LIST OF FIGURES}

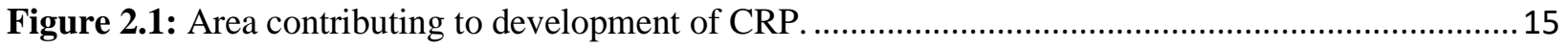

Figure 2.2: Molecular weight outcomes from ideal controlled radical polymerization...........................21

Figure 2.3: Molecular weight outcomes from an ideal controlled radical polymerization. ......................22

Figure 2.4: General polymer chain structure from controlled radical polymerization (Nabifar 2007)......23

Figure 2.5: Relative advantages and limitations of ATRP, NMRP and RAFT (Matyjaszewski, 2003)....26

Figure 4.1: 2L Stainless Steel Reactor System. …........................................................................ 43

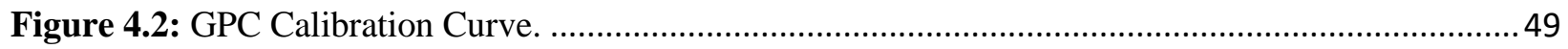

Figure 5.1: Synthesis of two-step emulsion polymerization of BMA in AGET ATRP...........................53

Figure 5.2: Aggregation/ Coagulation in AGET ATRP Emulsion System with HMTA as Ligand..........56

Figure 5.3: GPC traces for two-step emulsion polymerization with dNbpy ligand................................57

Figure 5.4: GPC traces for two-step emulsion polymerization preliminary investigation........................59

Figure 6.1: GPC traces of the effect of temperature at $50^{\circ} \mathrm{C}, 60^{\circ} \mathrm{C}$ and $70^{\circ} \mathrm{C}$ on BMA polymerization at 180min [BMA]:[EBiB]:[CuBr 2$]:[\mathrm{dNbpy}]:[\mathrm{AA}]:[$ Brij 98] = 219:1:0.11:0.21:0.17:5.18.

Figure 6.2: Conversion versus time for different reaction temperature $\left(50^{\circ} \mathrm{C}, 60^{\circ} \mathrm{C}\right.$ and $\left.70^{\circ} \mathrm{C}\right)$.

[BMA]:[EBiB]:[CuBr 2$]:[\mathrm{dNbpy}]:[\mathrm{AA}]:[$ Brij 98]= 219:1:0.11:0.21:0.17:5.18. 64

Figure 6.3: First order kinetics plot of BMA versus time for different reaction temperature $\left(50^{\circ} \mathrm{C}, 60^{\circ} \mathrm{C}\right.$ and $\left.70^{\circ} \mathrm{C}\right) .[\mathrm{BMA}]:[\mathrm{EBiB}]:\left[\mathrm{CuBr}_{2}\right]:[\mathrm{dNbpy}]:[\mathrm{AA}]:[\mathrm{Brij} 98]=219: 1: 0.11: 0.21: 0.17: 5.18$. 64

Figure 6.4: PDI versus conversion plot for different reaction temperature $\left(50^{\circ} \mathrm{C}, 60^{\circ} \mathrm{C}\right.$ and $\left.70^{\circ} \mathrm{C}\right)$.

[BMA]:[EBiB]:[CuBr 2$]:[\mathrm{dNbpy}]:[\mathrm{AA}]:[\mathrm{Brij} 98]=219: 1: 0.11: 0.21: 0.17: 5.18$. .65

Figure 6.5: Number average molecular weight vs conversion for different reaction temperature $\left(50^{\circ} \mathrm{C}\right.$, $60^{\circ} \mathrm{C}$ and $\left.70^{\circ} \mathrm{C}\right) .[\mathrm{BMA}]:[\mathrm{EBiB}]:\left[\mathrm{CuBr}_{2}\right]:[\mathrm{dNbpy}]:[\mathrm{AA}]:[\mathrm{Brij} 98]=219: 1: 0.11: 0.21: 0.17: 5.18$. .66

Figure 6.6: Particle size at $180 \mathrm{~min}$ at different temperature: $50^{\circ} \mathrm{C}, 60^{\circ} \mathrm{C}$ and $70^{\circ} \mathrm{C}$.

[BMA]:[EBiB]:[CuBr 2$]:[\mathrm{dNbpy}]:[\mathrm{AA}]:[$ Brij 98]= 219:1:0.11:0.21:0.17:5.18. 66

Figure 6.7: GPC traces of the effect of ligand concentration on BMA polymerization at 180min.

$[\mathrm{dNbpy}] /\left[\mathrm{CuBr}_{2}\right]=1,1.5$ and 3 respectively. [BMA]:[EBiB]:[CuBr 2$]:[\mathrm{AA}]:[\mathrm{Brij} 98]=213: 1: 0.12: 0.17: 5.15$,

Reaction temp $=70^{\circ} \mathrm{C}$.

Figure 6.8: Conversion versus time for different catalyst/ligand concentration.

$[\mathrm{dNbpy}] /\left[\mathrm{CuBr}_{2}\right]=1,1.5$ and 3 respectively. [BMA]:[EBiB]:[CuBr 2$]:[\mathrm{AA}]:[\mathrm{Brij} 98]=213: 1: 0.12: 0.17: 5.15$, Reaction temp $=70^{\circ} \mathrm{C}$. 70 
Figure 6.9: First order kinetics plot of BMA versus time for different catalyst/ligand concentration. $[\mathrm{dNbpy}] /\left[\mathrm{CuBr}_{2}\right]=1,1.5$ and 3 respectively. [BMA]:[EBiB]:[CuBr 2$]:[\mathrm{AA}]:[\mathrm{Brij} 98]=213: 1: 0.12: 0.17: 5.15$, Reaction temp $=70^{\circ} \mathrm{C}$ .70

Figure 6.10: PDI versus conversion plot for different reaction catalyst/ligand concentration.

$[\mathrm{dNbpy}] /\left[\mathrm{CuBr}_{2}\right]=1,1.5$ and 3 respectively. [BMA]:[EBiB]:[CuBr 2$]:[\mathrm{AA}]:[\mathrm{Brij} 98]=213: 1: 0.12: 0.17: 5.15$, Reaction temp $=70^{\circ} \mathrm{C}$.

Figure 6.11: Number average molecular weight vs conversion for different catalyst/ligand concentration. $[\mathrm{dNbpy}] /\left[\mathrm{CuBr}_{2}\right]=1,1.5$ and 3 respectively. [BMA]:[EBiB]:[CuBr 2$]:[\mathrm{AA}]:[\mathrm{Brij} 98]=213: 1: 0.12: 0.17: 5.15$, Reaction temp $=70^{\circ} \mathrm{C}$

Figure 6.12: GPC traces of the effect of surfactant on BMA polymerization at $180 \mathrm{~min}$. $\mathrm{Brij} 98 / \mathrm{H}_{2} \mathrm{O}=$ 3.81, 2.94 and 2.05wt\% respectively. BMA]:[EBiB]:[CuBr 2$]:[\mathrm{dNbpy}]:[\mathrm{AA}]=211: 1: 0.11: 0.21: 0.16$ Reaction temp $=70^{\circ} \mathrm{C}$. 74

Figure 6.13: Conversion versus time for different surfactant concentration. Brij $98 / \mathrm{H}_{2} \mathrm{O}=3.81,2.94$ and 2.05wt\% respectively. BMA]:[EBiB]:[CuBr 2$]:[\mathrm{dNbpy}]:[\mathrm{AA}]=211: 1: 0.11: 0.21: 0.16$ Reaction temp= $70^{\circ} \mathrm{C}$.

Figure 6.14: First order kinetics plot of BMA versus time for different surfactant concentration. Brij98/ $\mathrm{H}_{2} \mathrm{O}=3.81,2.94$ and $2.05 \mathrm{wt} \%$ respectively. BMA]:[EBiB]:[CuBr 2$]:[\mathrm{dNbpy}]:[\mathrm{AA}]=211: 1: 0.11: 0.21: 0.16$ Reaction temp $=70^{\circ} \mathrm{C}$. .75

Figure 6.15: PDI versus conversion plot for different surfactant concentration. Brij98/ $\mathrm{H}_{2} \mathrm{O}=3.81,2.94$

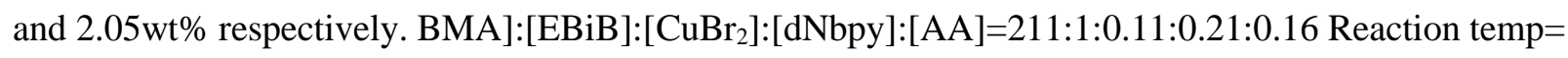
$70^{\circ} \mathrm{C}$.

Figure 6.16: Number average molecular weight vs conversion for different surfactant concentration. Brij98/ $\mathrm{H}_{2} \mathrm{O}=3.81,2.94$ and $2.05 \mathrm{wt} \%$ respectively.

BMA]:[EBiB]:[CuBr 2$]:[\mathrm{dNbpy}]:[\mathrm{AA}]=211: 1: 0.11: 0.21: 0.16$ Reaction temp $=70^{\circ} \mathrm{C}$. .77

Figure 6.17: GPC traces of the effect of reducing agent on BMA polymerization at 180min. $[\mathrm{Cu}(\mathrm{II})] /[\mathrm{AA}]=0.33,0.67$ and 1 respectively.

[BMA]:[EBiB]:[CuBr 2$]:[\mathrm{dNbpy}]:[\mathrm{Brij} 98]=205: 1: 0.10: 0.20: 4.87$, Reaction temp $=70^{\circ} \mathrm{C}$. .80

Figure 6.18: Conversion versus time for different reducing agent concentration. $[\mathrm{Cu}(\mathrm{II})] /[\mathrm{AA}]=0.33,0.67$ and 1 respectively. [BMA]:[EBiB]:[CuBr 2$]:[\mathrm{dNbpy}]:[\mathrm{Brij} 98]=205: 1: 0.10: 0.20: 4.87$, Reaction temp= $70^{\circ} \mathrm{C}$.

Figure 6.19: First order kinetics plot of BMA versus time for different reducing agent concentration. $[\mathrm{Cu}(\mathrm{II})] /[\mathrm{AA}]=0.33,0.67$ and 1 respectively.

[BMA]:[EBiB]:[CuBr 2$]:[\mathrm{dNbpy}]:\left[\right.$ Brij98] $=205: 1: 0.10: 0.20: 4.87$, Reaction temp $=70^{\circ}$. .81 
Figure 6.20: PDI versus conversion plot for different reducing agent concentration. $[\mathrm{Cu}(\mathrm{II})] /[\mathrm{AA}]=0.33$, 0.67 and 1 respectively. [BMA]:[EBiB]:[CuBr 2$]:[\mathrm{dNbpy}]:[$ Brij98]=205:1:0.10:0.20:4.87, Reaction temp= $70^{\circ} \mathrm{C}$. 81

Figure 6.21: Number average molecular weight vs conversion for different reducing agent concentration. $[\mathrm{Cu}(\mathrm{II})] /[\mathrm{AA}]=0.33,0.67$ and 1 respectively.

[BMA]:[EBiB]:[CuBr 2$]:[\mathrm{dNbpy}]:\left[\right.$ Brij98] $=205: 1: 0.10: 0.20: 4.87$, Reaction temp $=70^{\circ} \mathrm{C}$. .82 


\section{LIST OF TABLES}

Table 2.1: General Mechanism for a Conventional Free Radical Polymerization..................................17

Table 2.2: General Mechanism for a Controlled Radical Polymerization Process.................................19

Table 3.1: Most common AGET ATRP kinetics mechanism .............................................................. 36

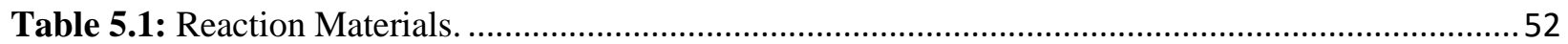

Table 5.2: Experimental conditions for emulsion AGET ATRP of BMA (Different Ligand System)...... 55

Table 5.3: Experimental condition for two-step emulsion AGET ATRP of BMA.................................58

Table 5.4: Experimental results for two-step emulsion AGET ATRP of BMA. ....................................59

Table 6.1: AGET ATRP emulsion polymerization using two-step technique for different temperatures. 62

Table 6.2: Experimental results of PBMA by a Two-step emulsion AGET ATRP using different temperatures.

Table 6.3: AGET ATRP emulsion polymerization using two-step technique for different ligand/catalyst ratios

Table 6.4: Experimental result of PBMA by a Two-step emulsion AGET ATRP of BMA using different ligand/catalyst ratios. .68

Table 6.5: AGET ATRP emulsion polymerization using two-step technique for different surfactant concentration.

Table 6.6: Experimental result of PBMA by a Two-step emulsion AGET ATRP using different surfactant concentration.

Table 6.7: AGET ATRP emulsion polymerization using two-step technique for different reducing agent concentration.

Table 6.8: Experimental result of PBMA by a Two-step emulsion AGET ATRP of BMA using different reducing agent concentration. 


\section{NOMENCLATURE}

$\begin{array}{ll}\text { AA } & \text { Ascorbic Acid } \\ \text { AGET } & \text { Activator Generated by Electron Transfer } \\ \text { ARGET } & \text { Activators Regenerated by Electron Transfer } \\ \text { ATRA } & \text { Atom Transfer Radical Addition } \\ \text { ATRP } & \text { Atom Transfer Radical Polymerization } \\ \text { BB } & \text { Band Broadening } \\ \text { BDE } & \text { Bond Dissociation Energy } \\ \text { BPMODA } & \text { Bis (2-pyridylmethyl) octadecylamine } \\ \text { BMA } & \text { Butyl Methacrylate } \\ \text { BPN } & \text { 2-bromopropionitrile } \\ \text { Bpy } & 2,2^{\prime}-\text { bypiridines } \\ \text { Brij } 98 & \text { Polyoxyethylene (20) oleyl ether } \\ \text { Conv } & \text { Conversion, \% } \\ \text { CRP } & \text { Controlled Radical Polymerization } \\ \text { Cu } & \text { Elemental Copper } \\ \text { Cu } & \text { Intermediate Copper } \\ \text { CuBr } 2 & \text { Copper Bromide } \\ \text { dNbpy } & \text { 4,4'-Dinonylbipyridine } \\ \text { DLS } & \text { Dynamic Light Scattering } \\ \text { DP } & \text { Degree of Polymerization } \\ \text { EBiB } & \text { Ethyl-2-bromoisobutyrate } \\ \text { FRP } & \text { Free Radical Polymerization } \\ \text { GPC } & \text { Initiator } \\ \text { HMW } & \text { HMTA } \\ \text { I } & \end{array}$




\begin{tabular}{|c|c|}
\hline ICAR & Initiators for Continuous Activator Regeneration \\
\hline $\mathrm{k}_{\mathrm{act}}$ & ATRP Activation Constant, $\mathrm{L} / \mathrm{mol} \mathrm{s}$ \\
\hline $\mathrm{K}_{\mathrm{ATRP}}$ & ATRP Equilibrium Constant, $\mathrm{L} / \mathrm{mol} \mathrm{s}$ \\
\hline $\mathrm{k}_{\text {deact }}$ & ATRP Deactivation Constant, L/mol s \\
\hline $\mathrm{k}_{\mathrm{t}}$ & Overall Termination Rate Constant, $\mathrm{L} / \mathrm{mol} \mathrm{s}$ \\
\hline $\mathrm{k}_{\mathrm{tc}}$ & Termination through coupling, $\mathrm{L} / \mathrm{mol} \mathrm{s}$ \\
\hline $\mathrm{k}_{\mathrm{td}}$ & Termination through disproportionation, $\mathrm{L} / \mathrm{mol} \mathrm{s}$ \\
\hline LALLS & Low-angle laser light scattering detector \\
\hline LMW & Low Molecular Weight \\
\hline M & Monomer \\
\hline$[\mathrm{M}]$ & Molar Concentration of Monomer, $\mathrm{mol} / \mathrm{L}$ \\
\hline$[\mathrm{M}]_{0}$ & Initial Monomer Concentration, $\mathrm{mol} / \mathrm{L}$ \\
\hline $\mathrm{MBrP}$ & 2-bromopropionate \\
\hline $\mathrm{Me}_{6} \mathrm{TREN}$ & $\mathrm{N}, \mathrm{N}, \mathrm{N}^{\prime}, \mathrm{N}^{\prime}, \mathrm{N}^{\prime \prime}, \mathrm{N}^{\prime}$-hexamethyl(tris(2-aminoethyl)-amine \\
\hline MMA & Methyl- Methacrylate \\
\hline MMD & Molar Mass Distribution \\
\hline $\mathrm{Mn}$ & Number Average Molecular Weight, g/mol \\
\hline $\mathrm{Mt}^{\mathrm{n}}$ & Transition Metal Species \\
\hline $\mathrm{Mt}^{\mathrm{n}} / \mathrm{L}$ & Transition Metal Complex (Low Oxidation State) \\
\hline $\mathrm{Mt}^{\mathrm{n}+1} / \mathrm{L}$ & Transition Metal Complex (High Oxidation State) \\
\hline MW & Average Molecular Weight, $\mathrm{g} / \mathrm{mol}$ \\
\hline MWD & Molecular Weight Distribution \\
\hline NMRP & Nitroxide-mediated Radical Polymerization \\
\hline PBMA & Polybutylmethacrylate \\
\hline PDI & Polydispersity Index (Mw/Mn) \\
\hline $\mathrm{Pr}_{\mathrm{r}}$ & Propagating radical of size $r$ \\
\hline $\mathrm{P}_{\mathrm{s}}$ & Propagating radical of size s \\
\hline $\mathrm{P}_{\mathrm{r}+\mathrm{s}}$ & Dead Polymers of Length $\mathrm{r}+\mathrm{s}$ \\
\hline
\end{tabular}




$\begin{array}{ll}\text { PEBr } & \text { 1-phenylethyl bromide } \\ \text { PMDETA } & \text { N, N,N',N",N"-pentamethyldiethylene-triamine } \\ \text { PRE } & \text { Persistent Radical Effect } \\ \text { PS } & \text { Polystyrene } \\ {[R \cdot]} & \text { Total Active Radical Concentration, mol/L } \\ \mathrm{R}_{\mathrm{i}}^{\cdot} & \text { Propagating Radical of Chain Length 1 } \\ \mathrm{R}_{\mathrm{in}} & \text { Primary Radical Generated from the Initiator } \\ \mathrm{R}_{\mathrm{r}} & \text { Propagating Radical of Size r, where } \mathrm{r} \geq 1 \\ \mathrm{R}_{\mathrm{s}}^{\cdot} & \text { Propagating Radical of Size s; where } \mathrm{s} \geq 1 \\ \mathrm{R}-\mathrm{X} & \text { Initiators } \\ \mathrm{RAFT} & \text { Reversible Addition-fragmentation Transfer Polymerization } \\ \mathrm{RALLS} & \text { Right-angle laser light scattering detector } \\ \mathrm{RI} & \text { Differential Refractor } \\ \mathrm{RP} & \text { Radical Polymerization } \\ \mathrm{r}_{\mathrm{t}} & \text { Rate of Termination } \\ \mathrm{S} & \text { Solvent } \\ \mathrm{SEC} & \text { Size exclusion chromatography } \\ \mathrm{SET}-\mathrm{LRP} & \text { Single-electron Transfer Living Radical Polymerization } \\ \mathrm{THF} & \text { Tetrahydrofuran } \\ \mathrm{t} & \text { Time, min, h } \\ \mathrm{T} & \text { Transfer Agent } \\ \mathrm{X} & \text { Halogen } \\ & \end{array}$




\section{CHAPTER 1}

\section{INTRODUCTION}

\subsection{Overview}

Synthetic polymers are essential components of various commodity and engineering materials in our daily life. Initially, polymers were dedicated for structural applications. Nowadays, numerous cutting-edge scientific developments in areas such as aeronautics, biomedical, nanotechnology and information technology rely substantially on the development of new polymeric materials with very specific molecular architectures. The increasing number of applications of synthetic polymer in higher functional materials explains why polymer science research is now considered as an essential and innovative research area in academia and industry (Nicolas and Guillaneuf, 2015).

In the field of polymer chemistry, the combined advantages of Free Radical Polymerization (FRP) and living ionic polymerization have allowed the feasible production of new polymers economically and the reason for the growing academic and industrial interest in Controlled Radical Polymerization (CRP). CRP method allows synthesis of a multitude of polymers, under relatively mild conditions, with good chemical architecture and narrow molecular weight distributions (Braunecker and Matyjaszewski, 2007).

There are three main types of processes covered under CRP: Atom transfer radical polymerization (ATRP), Reversible Addition-Fragmentation Transfer Polymerization (RAFT) and Nitroxidemediated Radical Polymerization (NMRP). Efforts have been undertaken to advance the knowledge of each process field in terms of chemical kinetics mechanisms, improvements of processes performance, and industrial applications. Currently, various CRP developments have significantly advanced in all these areas, but further improvements are still required, as evidenced in some recent papers (Destarac, 2010; Monteiro and Cunningham, 2012; Oh, 2008, Pintauer et al., 2016; Ribelli et al. 2018, Surmacz and Chmialarz, 2020). Several studies have fostered a deeper understanding of the CRP processes in different media. 
Among the three main CRP, ATRP is the most popular polymerization technique in both academia and industrial settings. ATRP process attracts industrial interest because it is robust, easy to set-up and the commercial availability of initiators and catalysts. Polymers synthesized using ATRP techniques, can be used in various applications such as lubricants, adhesives, additives, thermoplastic elastomers, as well as in biomedical applications like bioconjugates, drug delivery and artificial bones (Braunecker and Matyjaszweski, 2007; Destarac, 2010; Matyjaszweski, 2014, Awad et al., 2020). In addition, polymers synthesized through ATRP are being evaluated for their commercial applications and their development in aqueous dispersed media. Besides, ATRP in aqueous dispersed media is economically and environmentally attractive because water is an environmentally friendly and inexpensive reaction medium that provides efficient heat transfer and less viscous reaction media.

However, conducting ATRP in aqueous dispersed media is not quite straightforward as homogeneous polymerization system. Challenges had to be overcome to achieve control over molecular structures and colloidal stability when ATRP was conducted in aqueous dispersed media. Consequently, the present research work will attempt to expand some of characteristics at play in AGET (Activator Generated by Electron Transfer) ATRP system. AGET ATRP is selected in this study, due to its ability to generate high purity block copolymers, its good end group functionality, and large range of applicable monomers. This system will be studied through experimental investigation of Activators Generated by Electron Transfer Atom Transfer Radical Polymerization (AGET ATRP) of Butyl Methacrylate in a two-stage dispersed system.

\subsection{Objectives}

The objective of this thesis is to provide further insight into the AGET ATRP process. To accomplish this goal, an experimental investigation was carried out using two-step emulsion polymerization of butyl methacrylate (BMA) under different conditions:

- Determine favourable experimental conditions under which AGET ATRP polymerization can be performed in a $2 \mathrm{~L}$ reactor.

- Collect experimental data, which provide information about the nature of AGET ATRP process in dispersed media. 
- Provide quantitative insight into the behavior of the AGET ATRP in emulsion.

- Investigate the reactor's performance for different factors: temperature, surfactant, catalyst/ligand, and reducing agent.

\subsection{Thesis Organization}

- Chapter 2 provides background information and literature review of Free Radical Polymerization and Controlled Radical Polymerization.

- Chapter 3 provides an overview and literature review of Atom Transfer Radical Polymerization.

- Chapter 4 presents the laboratory-scale experimental set-up of the stirred tank reactor system and polymer characterization methods.

- Chapter 5 discusses the preliminary experimental work done on AGET ATRP of BMA in a two-stage dispersed system. An experimental design was set-up to develop a sound analysis of the BMA AGET ATRP process. A quantitative analysis is done in this study.

- Chapter 6 discusses the experimental work to understand the effects of various factors in AGET ATRP process. The research focus in this chapter is to interpret the experimental data trends.

- Chapter 7 summarizes the research findings and propose recommendations. 


\section{CHAPTER 2}

\section{FREE RADICAL POLYMERIZATION}

\subsection{Background}

Among all the crucial contribution in polymer science, the development of living polymerization by Michael Szwarc in 1956 who studied anionic polymerization of styrene in tetrahydrofuran was a significant milestone. His discovery facilitated major development in areas of synthetic polymer chemistry as it opened the avenue to the synthesis of well-defined polymers with tailor-made macromolecules and nano-structured morphologies (Braunecker and Matyjaszweski, 2007). His innovation contributed to the foundation of modern nanotechnology. The basis of living polymerization is the occurrence of chain growth polymerization under the absence of conventional bimolecular termination or any irreversible transfer reactions, through the establishment of a dynamic equilibrium between the active and dormant species (Fischer, 1999 and Fischer, 2001). Hence, such method can produce narrowly distributed living polymer. Living polymerization technique exhibits two major drawbacks: (i) limitation to polymerize a range of monomer due to a lack of compatibility to active centre in certain functional groups and (ii) requirement of stringent reaction condition and removal of impurities (i.e. air and traces of water) (Nicolas et al., 2013).

Living anionic and cationic polymerizations were the first types of living process for several decades after its realization. The free radical polymerization (FRP) differs from ionic polymerization by: (i) significantly lower sensitivity to impurities; (ii) wider range of monomer compatibility including (meth)acrylates, (meth)acrylamides, acrylonitrile, styrenic derivatives, dienes and vinyl monomers; (iii) higher number of polymerization processes can be implemented (i.e. bulk, solution, emulsion, dispersion, etc.); (iv) higher tolerance of unprotected functionality in monomer and solvent (e.g. $\mathrm{OH}, \mathrm{NR}_{2}, \mathrm{COOH}, \mathrm{CONR}_{2}$, and $\mathrm{SO}_{3} \mathrm{H}$ ); (v) greater simplicity and easiness to implement and relatively inexpensive to competitive technology (Nicolas et al., 2013). In addition, free radical polymerization is very attractive and profitable in the production of materials with sophisticated structures and architecture (i.e. blocks and gradient copolymers; star, comb, and hyperbranched architectures). However, the main drawback of free-radical 
polymerization is the lack of chemical controllability over the synthesized materials molar mass, molar mass distribution (MMD), the chain-end functionalities and macromolecular architecture (Nicolas and Guillaneuf, 2015). Therefore, a new field of macromolecular synthesis was devised upon combining the easiness of free-radical polymerization with the high standard controllability of living ionic polymerization (Figure 2.1) into a single polymerization process (Nabifar, 2007).

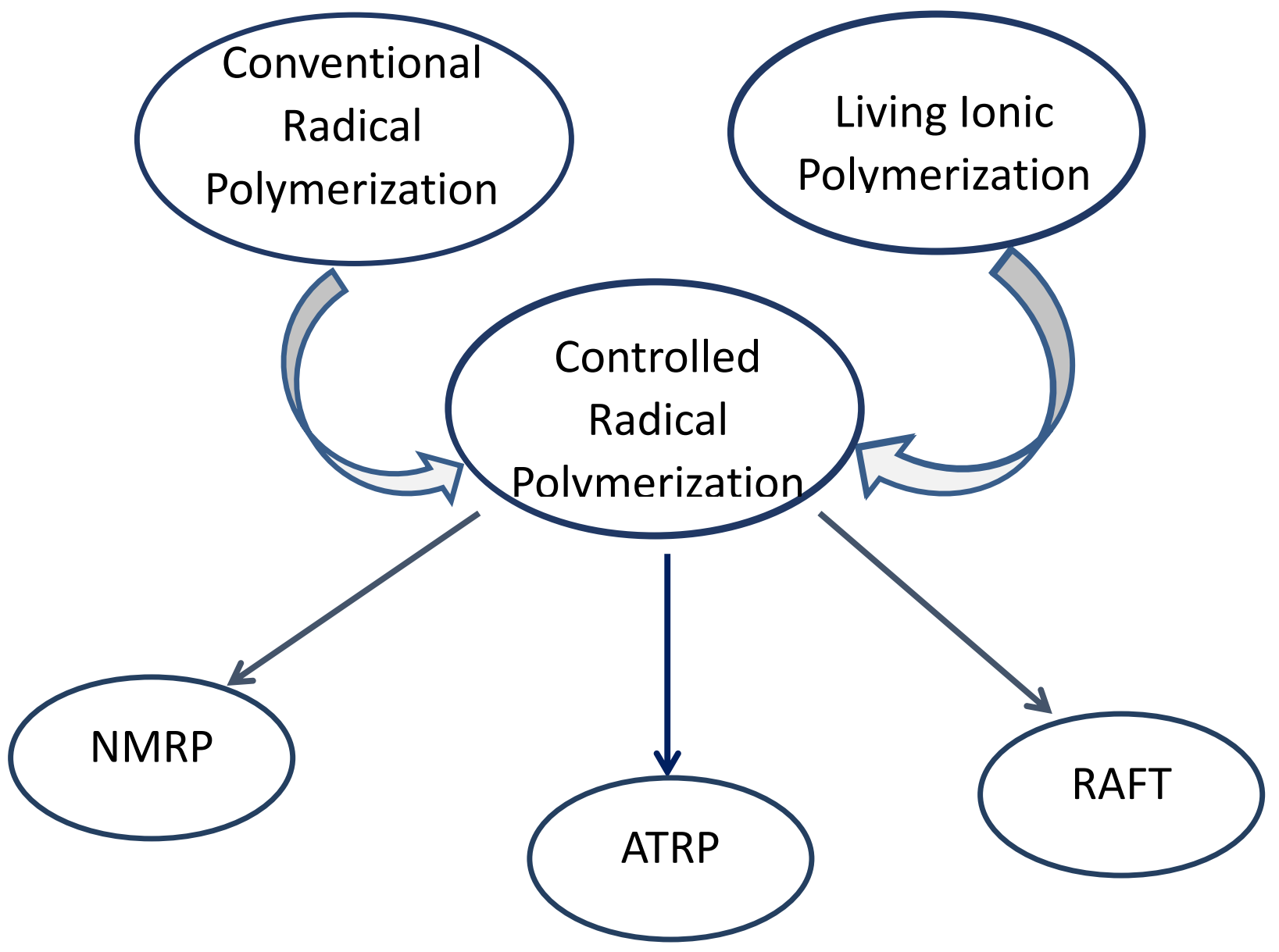

Figure 2.1: Area contributing to development of CRP.

In this perspective, CRP provides an alternative to FRP as it can produce synthetic polymers with narrow molecular weight distribution (MWD) and ensures simultaneous growth of chain and suppression of bimolecular termination (Mastan et al., 2015). However, a common drawback with $\mathrm{CRP}$ is that high molecular weight product with good mechanical properties is barely attainable (Tuinman et al., 2006). 


\subsection{Conventional Radical Polymerization: Free Radical Polymerization}

Since controlled radical polymerization contains the elementary radical reactions found in conventional free radical polymerization (FRP), it is necessary to comprehend the fundamental mechanism of FRP first in order to understand that of CRP.

Free radical polymerization is the widely used polymerization techniques. It is classified under the polymerization subgroup termed chain-growth polymerization, which shares a relatively common feature with other chain-growth polymerization reactions. The mechanism can be broken into three elementary parts: initiation, propagation, and termination. In addition, radical polymerizations tend to have a transfer reaction in which an active radical can transfer its activity to another molecule (Louie et al., 1985). As shown in Table 2.1, the first reaction step is the initiation, which involves two reactions: generation of primary radicals $\left(\mathrm{R}^{*}\right.$ in $)$ through homolytic dissociation of an initiator species and reaction of these radicals with monomer to produce radicals of chain length unity $\left(\mathrm{R}_{1}{ }_{1}\right)$. Typical initiators (peroxide, azo compounds, etc.) are used with very low concentrations between 0.01-1 mol\%.

The second step is a propagation reaction, which consists of the growth of polymer radical species $\left(\mathrm{R}_{\mathrm{r}}{ }_{\mathrm{r}}\right)$ through successive additions of monomer molecules. Each propagation (chain growth) reaction expands the relevant polymer chain randomly by one monomer unit. Thus, the propagation reaction is randomly repeated and causes the polymer chains to grow. The propagation rate constant is assumed chain length independent, with typical values of $\mathrm{k}_{\mathrm{p}} \sim 10^{3} \mathrm{M}^{-1} \mathrm{~s}^{-1}$ (Braunecker and Matyjaszewski, 2007).

At some point, the propagating radical stops growing and terminates. The termination with annihilation of polymer radical centres occurs by bimolecular reactions between two polymer radicals. Termination of two radicals can be by either coupling $\left(\mathrm{k}_{\mathrm{tc}}\right)$ or disproportionation $\left(\mathrm{k}_{\mathrm{td}}\right)$.

Chain transfer reaction is a reaction in which the growth of a live polymer chain is terminated by abstraction of a labile hydrogen atom from a smaller molecule. As a result, the live polymer radical centre is transferred to the smaller molecule (i.e. monomer, solvent, chain transfer agent) and the live polymer chain becomes a dead polymer chain. However, since the radical is transferred to another molecule, which can further re-initiate and propagate; it implies that the overall radical 
concentration is not reduced. Thus, chain transfer is not a chain terminating reaction. Shown below in Table 2.1 is the general mechanism for the free radical polymerization process:

Table 2.1: General Mechanism for a Conventional Free Radical Polymerization.

Initiation

$$
\begin{aligned}
& I \stackrel{k d}{\rightarrow} 2 R_{\text {in }}^{\cdot} \\
& R_{\text {in }}^{\cdot}+M \stackrel{k_{p}^{\prime}}{\rightarrow} R_{1}^{\cdot}
\end{aligned}
$$

Propagation

$$
R_{r}^{\cdot}+M \stackrel{k_{p}}{\rightarrow} R_{r+1}^{\cdot}, \quad r \geq 1
$$

Termination

$$
\begin{aligned}
& R_{r}^{\cdot}+R_{s}^{\cdot \stackrel{k t c}{\longrightarrow}} P_{r+s} \\
& R_{r}^{\cdot}+R_{s}^{\cdot} \stackrel{k t d}{\longrightarrow} P_{r}+P_{s}
\end{aligned}
$$

Chain transfer

$$
R_{r}^{\cdot}+H-T \stackrel{K_{t r}}{\longrightarrow} P_{r}+T^{\bullet}
$$

This Table gives the general mechanism for conventional free radical polymerization process. This mechanism only includes the main reactions commonly involved in this process. Where $I$ : Initiator, $R_{i n}^{*}$ : primary radical generated from the initiator, $M$ : monomer, $T$ : transfer agent, $R_{r}^{\bullet}$ : propagating radical of size $r$, where $r \geq 1, R_{s}^{*}$ : propagating radical of size $s$, where $s \geq 1$, $P_{r+s} / P_{r} / P_{s}$ : are the dead polymers of length $r+s, r$ and $s$ respectively (Braunecker and Matyjaszewski, 2007; Matyjaszewski and Tsarevsky, 2014; Nicolas et al., 2013).

In regular radical polymerization, all four main steps (initiation, propagation, termination, and transfer) occur concurrently. Initiation is required to occur throughout the polymerization in order to maintain a relatively constant (steady state) radical concentration, since termination of radical species are occurring throughout the polymerization. The overall time for a chain to be initiated, propagate, and then terminate is within the order of seconds. The time taken to consume all the monomer normally ranges between minutes to hours. The selection of a proper initiator is 
important as it decomposes into primary radicals throughout the reaction (Dhib et al., 2000; Nicolas et al., 2013; Nabifar, 2007; Odian, 2004). This method has several consequences:

- At the early stage in the reaction, high molecular weight materials are formed. However, chains that are produced early in the reaction are likely to have a different degree of polymerization in comparison to those produced later in the reaction (Nicolas et al, 2013). Thus, the molecular weight distribution (MWD) of the final polymer contains a wide range of polymer chains of different PDI (polydispersity index). As a result, the minimum PDI (ratio of the weight average to number average molecular weight $M_{w} / M_{n}$ ) that can be obtained in a regular free radical polymerization is 1.5 (Odian, 2004). However, the PDI is greater than 1.5 in most cases and it is often in the range of 2 to 3 or even higher.

- There is almost no control over chain end-groups.

With the understanding of the free radical polymerization mechanism, some of the major advantages and disadvantages of the process can be pointed out. Some of the advantages in free radical polymerization are the ability to form high molecular weight material relatively quick. FRP is relatively fast polymerization process and has large monomer applicability. On the other hand, FRP still suffers some significant disadvantages such as radicals losing their functionality due to irreversible termination, the reaction has poor stereo-selectivity and little control over the individual polymers or their population is usually attained (Braunecker and Matyjaszewski, 2007).

\subsection{Controlled Radical Polymerization (CRP)}

Development of several controlled/living radical systems has proposed since early 1980, with variations in mechanistic approaches. All these polymerization processes have a common mechanistic key feature, which is the establishment of dynamic equilibrium between the propagating radicals, and dormant species throughout the polymerization (Bergenudd, 2011; Chan et al., 2013; Matyjaszewski and Xia, 2001). The corresponding propagating live polymer radicals may either be reversibly trapped in a deactivation/activation or they can be terminated with other growing radicals.

In CRP process, the macromolecular synthesis exhibits features of living polymerization where the occurrence of chain breaking reactions is minimized and the apparent simultaneous growth of 
all the chains is achievable through almost instantaneous initiation (Braunecker and Matyjaszewski, 2007). A combination of fast initiation and reduction of irreversible termination reactions to an extremely low level seemingly conflicts with the fundamental principles of RP (Aldabbagh et al., 2008). In typical reaction system, the rate of radical-radical termination is high. As a result, radical lifetime is short and polymer chains are essentially dead at any given instant (Braunecker and Matyjaszewski, 2007). During these time frames, it is not possible to have low polydispersity and desirable chain end functionality (Barner-Kowollik et al., 2002). Shown below in Table 2.2 is the general mechanism for the controlled radical polymerization process:

Table 2.2: General Mechanism for a Controlled Radical Polymerization Process.

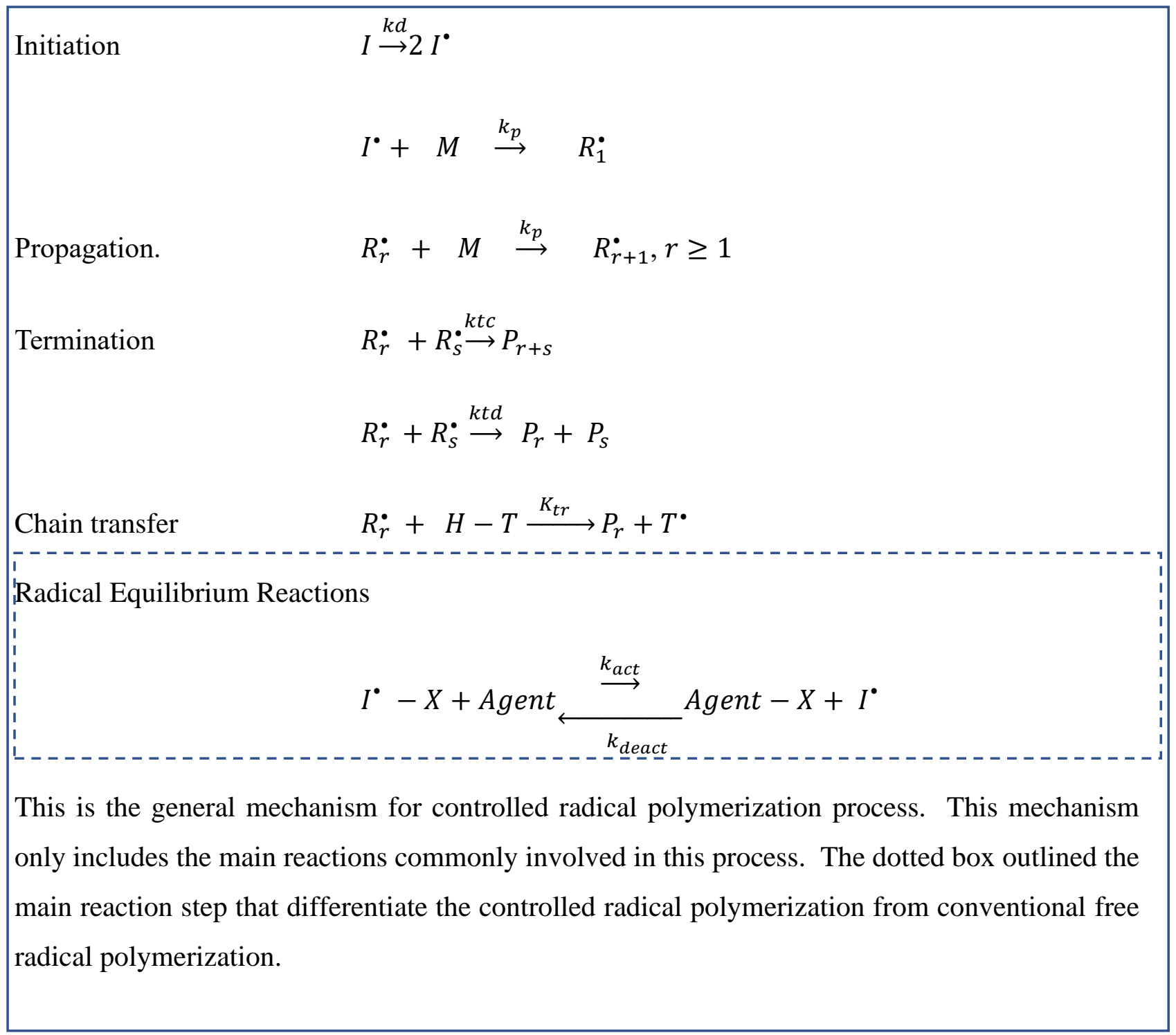


The variables used above are defined as: $I$ : Initiator, $I^{\bullet}$ : primary radical generated from the initiator, $M$ : monomer, $T$ : transfer agent, $R_{r}^{\bullet}$ : propagating radical of size $r$, where $r \geq 1, R_{s}^{\bullet}$ : propagating radical of size $s$, where $s \geq 1, P_{r+s} / P_{r} / P_{s}$ : are the dead polymers of length $r+s, r$ and $s$ respectively. Agent: refer to the different chemicals depending on the controlled radical polymerization initiation method used. $\mathrm{K}_{\mathrm{ATRP}}=\mathrm{k}_{\mathrm{act}} / \mathrm{k}_{\text {deact }}$ (Barner-Kowollik et al., 2002; Bergenudd, 2011; Braunecker and Matyjaszewski, 2007; Shipp, 2005).

In order to obtain a typical living polymerization using a radical intermediate, it is necessary to keep the concentration of growing chains at an appropriate level (somewhere around $10^{-4}$ to $10^{-1} \mathrm{M}$ ), while keeping the active radical concentration preferably less than $10^{-8} \mathrm{M}$ (Shipp, 1998). Consequently, the rate of bimolecular termination is given by:

$$
r_{t}=k_{t}[R \cdot]^{2}
$$

where $r_{t}$ is the rate of termination, $k_{t}$ is the termination rate constant and $[R \cdot]$ is the total active poly radical concentration.

The common method to achieve a good living polymerization is through capping the end of active radicals with a non-radical moiety (stable radical) that can be easily removed to yield back a radical (Braunecker and Matyjaszewski, 2007; Nabifar, 2007). Ideally, most chains at a given time are in dormant stage and only a small concentration $\left(\sim 10^{-7}\right.$ to $\left.10^{-9} \mathrm{M}\right)$ may have propagating radicals located at the chain end ( $\mathrm{Li}$ and Matyjaszewski, 2002; Nabifar, 2007). Furthermore, the stable radical can be easily broken down and hence reactivate the dormant chains, and alternatively the stable radical can react with an active chain and render it dormant. As a result, a fast equilibrium between the dormant and active chains is established and then released. The selection and characteristics of the stable radical is critical for the success of controlled radical procedure.

The use of capping group is not enough to gain low polydispersity. The equilibrium reaction between the dormant and active species must not only favour the dormant species but it must also be fast (Li and Matyjaszewski, 2002); which means that in each activation/deactivation cycle, stable radicals must leave and rejoin active radicals at a rate fast enough to allow few propagation 
steps. If the activation and deactivation reaction are relatively faster than the propagation, this implies that the polymer radicals will grow in an incremental fashion. In addition, if all chains are initiated at the same time, the resulting chains should have low polydispersity.

\subsubsection{General Considerations of CRP}

The ideal CRP system should have the following features:

1. First order kinetic behavior

There should be a linear kinetic behaviour of $\ln [\mathrm{M}]_{\mathrm{o}} /[\mathrm{M}]$ with time for an isothermal batch reactor. With instantaneous initiation and no termination, constant propagating radical is established and results in a linear trend of $\ln [\mathrm{M}]_{\mathrm{o}} /[\mathrm{M}]$ versus time, as shown in Figure 2.2. The curvature of the plot illustrates a deviation from the ideal situation caused by a slow initiation, loss of polymer radicals to termination reaction or other side reactions.

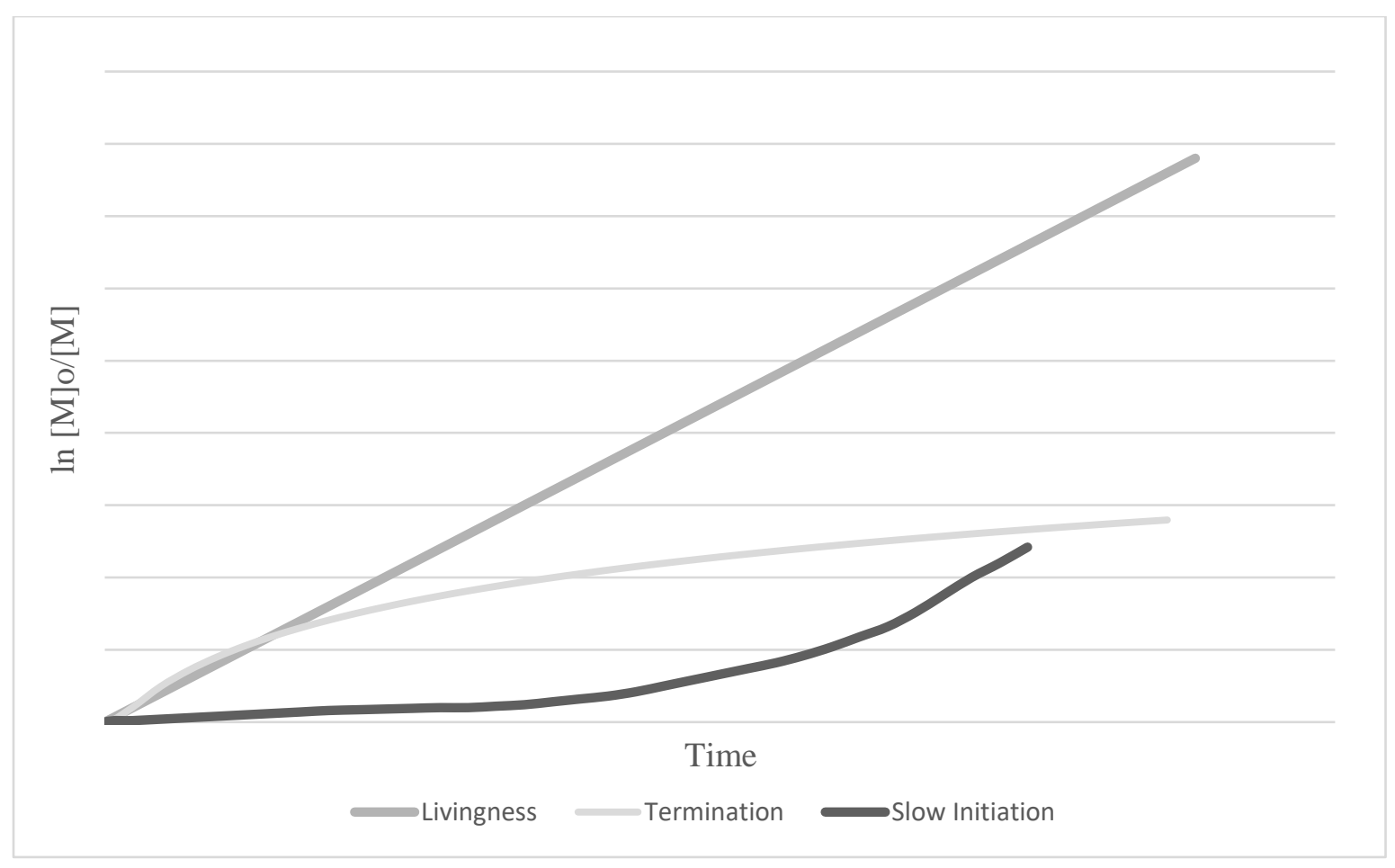

Figure 2.2: Molecular weight outcomes from ideal controlled radical polymerization. 
2. Linear increase in average molecular weight with conversion

There is a linear increase of the number-average molar mass $\left(\mathrm{M}_{\mathrm{n}}\right)$ with monomer conversion, which is not achievable in free radical polymerization (RP) where high molecular weights are produced immediately at the reaction onset as shown in Figure 2.3 (Braunecker and Matyjaszewski, 2007). Ideally, systems that are chemically controlled, lead to the formation of polymer chains with degree of polymerization (DP: number of monomer repeat units in a chain) predetermined by the ratio of concentration of the consumed monomer to the initial initiator concentration, which is given below:

$$
D P_{n}=\frac{\Delta[\mathrm{M}]}{[I]_{o}}
$$

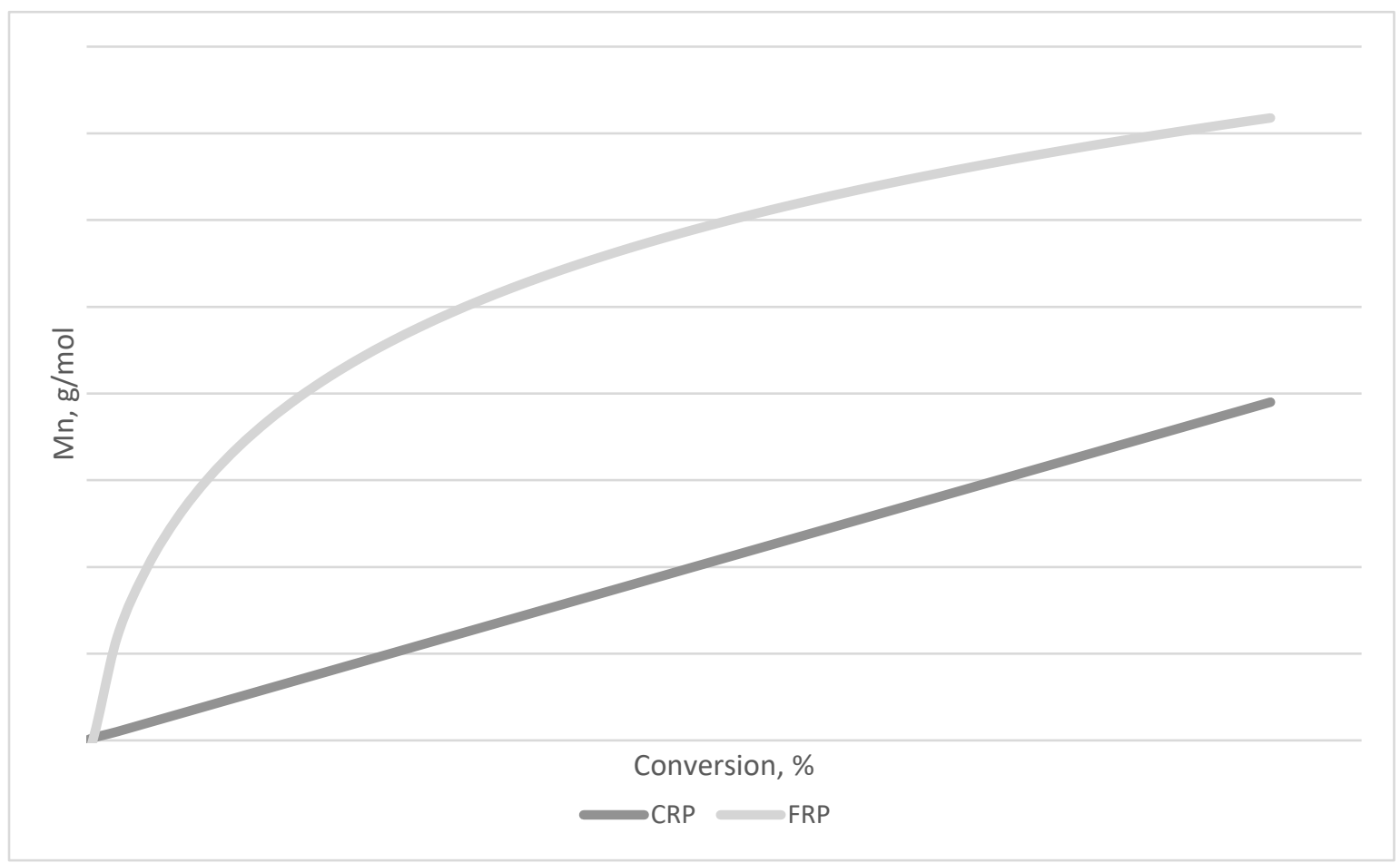

Figure 2.3: Molecular weight outcomes from an ideal controlled radical polymerization. 
3. Narrow molecular weight distribution:

Polydispersity indexes (PDI) defined as $\mathrm{M}_{\mathrm{w}} / \mathrm{M}_{\mathrm{n}}$ where $\mathrm{M}_{\mathrm{w}}$ is the weight-average molar mass. In addition, the target value of PDI value in CRP system should be less than 1.5 (the lowest limit for a conventional radical polymerization). Absence of both termination and chain transfer reactions is not the only reason to achieve low PDI. However, there are other reasons to get low PDI such as (Coleman and Fox, 1963; Nabifar, 2007; Matyjaszewski, 2017):

- Almost instantaneous initiation of all the chains producing simultaneous growth of active polymer chains;

- Reaction rate between the active and dormant species is quite higher compared to the rate of propagation;

- Chain transfer and termination effects are negligible;

- The rate of depropagation is assumed negligible in comparison with the rate of propagation.

4. Extended lifetime of live polymer chains with preserved chain-end functionalities:

The participation of the reversible activation elongates the average lifetime of growing chains from $\sim 1 \mathrm{~s}$ in FRP to over more than 1hr in CRP (Nabifar, 2007). Reversible activation combined with minimal termination leads to the observed narrow molecular weight distribution. In addition, the nature of the reaction mechanism enables specific end functionalization (Figure 2.4) or an addition of a second monomer to make a block copolymer, which is unlikely to occur in a conventional RP system.

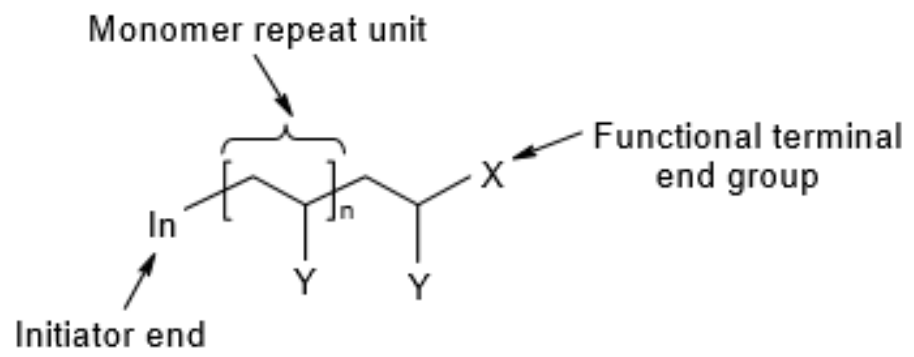

Figure 2.4 General polymer chain structure from controlled radical polymerization (Nabifar 2007). 
5. The possibility for polymer chains to grow again when additional monomer is introduced, allowing block copolymers to be synthesized (Braunecker and Matyjaszewski, 2007).

\subsubsection{Comparison of FRP with CRP}

In general, FRP and CRP follow similar reaction mechanism and can polymerize a similar range of monomers. However, the differences between CRP and FRP still exist as summarized in the followings (Braunecker and Matyjaszewski, 2007):

- The lifetime of growing polymer chains in FRP is very short and the monomer is consumed until depletion. However, this phenomenon has not been observed in the CRP process due to the participation of dormant species and intermittent reversible activation-deactivation scheme (Nicolas and Guillaneuf, 2015).

- Initiation in FRP is slow and some primary initiator radicals are sometimes left unconsumed. However, initiation in CRP systems is very fast, and instantaneous growth for all the chains is possible, which enables a control over chain architecture.

- Termination occurred between long chains and new chains are usually generated in FRP. In CRP systems, due to persistent radical effect (PRE) chains are short at the early stages of the reactions and become progressively longer overtime. As a result, termination rate significantly decreases with time.

\subsection{Controlled Radical Polymerizations}

\subsubsection{Types of CRP}

Currently, there are three main types of processes covered under CRP: Atom Transfer Radical Polymerization (ATRP), Reversible Addition-Fragmentation Transfer polymerization (RAFT) and Nitroxide-Mediated Radical Polymerization (NMRP).

ATRP and NMRP are both governed by persistent radical effect (PRE). Persistent radical is a peculiar kinetic phenomenon that provides self-regulating effect, where propagating radicals are reversibly trapped in a deactivation process. Propagating radicals are unlikely to terminate with each other, but they may undergo irreversible self-termination. Hence, any occurrence of selftermination can decrease the concentration of the propagating radical species and consequently 
leads to a slow accumulation of persistent species (Nicolas et al., 2013). In the systems obeying PRE, fast and almost simultaneous initiation of all chains is necessary for low MWD or PDI (Ayres, 2011; Braunecker and Matyjaszewski, 2007; Chaivin et al., 2006; Finke, 1992; Fischer, 1999; Goto and Fukuda, 1999; Upadhayay et al., 2017)

On the other hand, RAFT obeys a degenerative transfer rather than PRE. A steady state of growing radicals is established by initiation and termination. Nevertheless, RAFT follows typical FRP kinetics with slow initiation and fast termination (Apostolovic et al., 2006; Barner-Kowollik et al., 2002; Matyjaszewski and Xia, 2001; Zhang and Ray, 2001).

In ATRP, kinetics and chain control depend on both persistent radical and activator (lower oxidation state metal complex), whereas NMRP mostly depends on persistent radical (Nitroxide) and RAFT depends on transfer agent.

ATRP systems require specific initiator that are different from conventional radical initiators (peroxides and diazo compounds). In addition, ATRP is mediated by transition metal complex that requires ligands for reaction stability. Initiators are usually haloalkanes with halogens atom usually in secondary or tertiary carbons (Upadhayay, 2016). Commonly, a higher oxidation state metal complex acts as a persistent radical. However, NMRP and RAFT use conventional radical initiators. NMRP system is mediated by nitroxide radical which acts as persistent radical, whereas RAFT is mediated by a transfer agent (which is not a persistent radical). Recent development on ATRP system employs a reducing agent in the reaction initiation in order to reduce the amount of catalyst required. Ligands and reducing agents are not found in NMRP and RAFT.

Unfortunately, transition metal complex (mostly copper compounds) utilized in ATRP are toxic to environment. Removing it from the final product is the major drawback of ATRP system. Similarly, sulphur compound used as transfer agent in most RAFT also constitute a major problem. On the other hand, high bond dissociation energy of alkoxyamine (dormant species) needs higher temperature $\left(\geq 100^{\circ} \mathrm{C}\right)$ which is the major problem in NMRP (Moad and Solomon, 2006; Nabifar, 2007). Range of polymerizable monomers is quite high in RAFT followed by ATRP and then NMRP. Monomers with acidic functional groups are difficult to polymerize by ATRP, whereas methacrylate monomers are difficult to polymerize by NMRP (Upadhayay, 2016). One good advantage of ATRP is that it can produce clean block copolymers which is not possible in RAFT. Furthermore, it is not easy to introduce chain end functionality in NMRP and RAFT as compared 
with ATRP (Matyjaszewski, 2003). Higher commercial availability and more choice of reagents in ATRP are distinct advantages of ATRP over NMRP and RAFT.

Each of the three CRP has its own advantages and disadvantages as depicted in Figure 2.5, which shows low molecular weight (LMW), high molecular weight (HMW), range of polymerizable monomers (Mon Range), block copolymers (Blocks), end-functional polymers (End Funct.), hybrids, aqueous systems (water) and some environmental issues (Env). For example, ATRP is good for synthesizing block copolymers, polymers with functional groups and low molecular weight polymer. It is also a better method for incorporating inorganic compound into polymer chains. RAFT can be used with the widest range of monomers and it is a good method for high molecular weight polymers. In addition, RAFT is the most applicable method in aqueous medium. On the other hand, NMRP is the method that causes the least environmental impact.

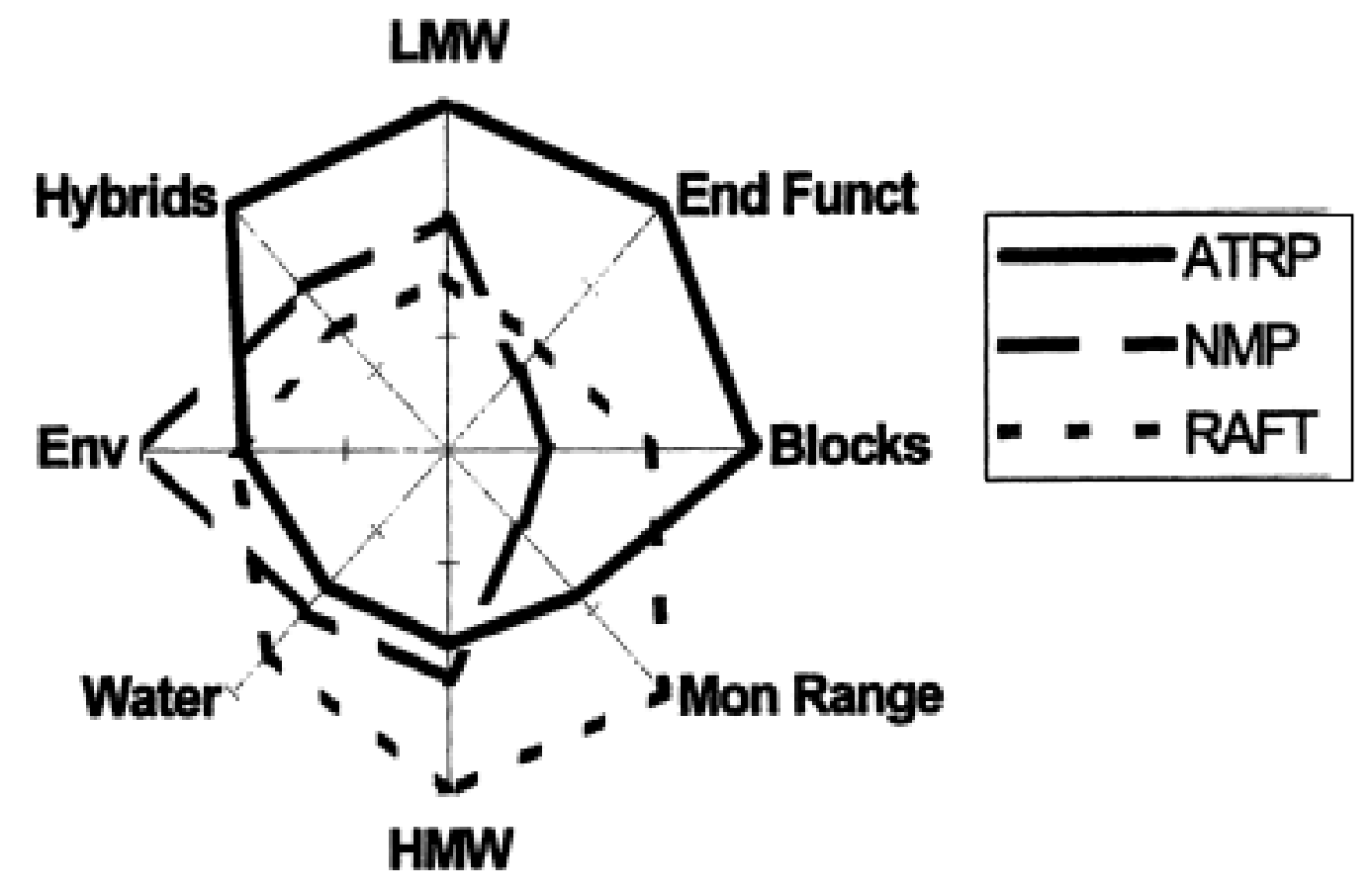

Figure 2.5: Relative advantages and limitations of ATRP, NMRP and RAFT (Matyjaszewski, 2003).

\subsubsection{Reason for Selecting ATRP over NMRP and RAFT}

ATRP can be carried out at mild temperature in comparison with NMRP (Moad and Solomon, 2006; Nabifar, 2007). Monomer compatibility with ATRP system is higher than with NMRP 
systems (Matyjaszewski, 2003). Disubstituted alkenes (like methyl methacrylate) are easy to polymerize by ATRP (Bertin et al., 2004; Chauvin et al. 2006; Upadhayay, 2016).

ATRP allows production of clean block copolymers which may not be easily achievable with RAFT. ATRP does not required transfer agents which involve unwanted sulphur compound that is essential in most RAFT methods. End group functionalization in ATRP is much easier and almost perfect as compared to RAFT or NMRP (Upadhayay, 2016). Very cheap reagents are highly available in ATRP as compared to NMRP and RAFT.

However, ATRP has limitations too: acidic monomers such as vinyl acetate having transferrable hydrogen atoms are difficult to polymerize. The need to use toxic transition metal complex (mostly copper compound) is not environmentally favourable.

Fortunately, several ATRP initiation methods have been developed to reduce the cost of purifying the final product by using low concentration metal catalyst (Kreutzer, 2018; Shen et al., 2004; Surmacz and Chmielarz, 2020). The decrease of catalyst concentration is beneficial not only for economic and environmental perspectives, but also for minimizing catalyst side reactions with propagating radicals (Krys and Matyjeszewski, 2017). Also, photoinduced metal free ATRP has been recently developed and can lead ATRP towards the preparation of polymers for biological, microelectronics and other metal sensitive applications (Upadhayay, 2016). Significant development of new synthesized ligands, enhancing the reactivity of metal complex, has been realized to facilitate polymerization in dispersed media. Currently, progress has been made in polymerizing a number of polar monomers. The number of studies done on different CRP technique until 2020 is in the order ATRP $>$ RAFT $>$ NMRP, which indicates that ATRP is more popular than RAFT and NMRP techniques (Matyjaszewski, 2017; Surmacz and Chmielarz, 2020; Tsarevsky and Sumerlin, 2013). 


\section{CHAPTER 3}

\section{ATOM TRANSFER RADICAL POLYMERIZATION}

\subsection{Overview}

In 1995, two research groups independently reported a similar new controlled radical polymerization technique, which was named 'Atom Transfer Radical Polymerization' or simply ATRP. This technique was based on a catalytic system used for the atom transfer radical addition reaction (ATRA), also known as Kharasch reaction. It is an efficient method of forming carboncarbon bonds between organic halides and alkenes. Sawamoto et al. (1995). This was first reported to polymerize MMA initiated by $\mathrm{CCl}_{4}$ using $\mathrm{RuCl}_{2}\left(\mathrm{PPh}_{3}\right)_{3} / \mathrm{Al}(\mathrm{O}-\mathrm{iPr})_{3}$ as a catalyst.

Wang and Matyjaszewski (1995) then reported the polymerization of styrene catalyzed by $\mathrm{CuCl} / 2,2$-bipyridyl (bpy) in the presence of 1-phenylethyl chloride as an initiator. Since then, there has been emergence of successive reports with regards to ATRP of styrene, acrylates, methacyrlates and acrylonitrile, using various transition metal complexes, such as nickel, iron, palladium, and rhodium (Destarac, 2010). In comparison with other controlled radical polymerization methods, ATRP was found to be very versatile. This technique provides controlled polymerization of many monomers under various reaction conditions and it can prepare polymers having a wide range of architectures including blocks, grafts, gradient copolymers, stars, combs, branched and hyper-branched. In addition, ATRP can be carried out in bulk, organic solvents, and heterogeneous aqueous systems. However, in nearly all cases, the ATRP reaction is not applicable in pure water for hydrophilic monomers due to side reactions (Destarac, 2010).

In early years of ATRP development, setbacks for the production of large volumes of materials through ATRP were encountered due to large volume of catalyst required to control the polymerization. For both economical and environmental perspectives, the level of potentially hazardous catalyst in the polymer material had to be strongly reduced for ATRP polymer to be accepted in marketplace (Matyjaszewski and Tsarevsky, 2013). ATRP catalyzed by copper/ligand complex has been extensively studied in academia and aroused interest in industry. Over the last three decades, significant improvement has been made either by increasing the catalytic activity of the $\mathrm{Cu}$ complexes or by recycling and recovering of the $\mathrm{Cu}$ complex. In most cases, about 100- 
200ppm of copper are still needed in regular ATRP (Surmacz and Chmielarz, 2020). In spite of the applauded research development in ATRP, availability of the best ligands for the preparation of highly active $\mathrm{Cu}$ catalyst are still lacking. For instance, 2-2'-bypiridine is largely produced, but the activity complex of $\mathrm{Cu} / \mathrm{bpy}$ is too low and requires high metal concentrations (Destarac, 2010; Matyjaszewski and Tsaversky, 2014). PMDETA is a much better $\mathrm{Cu}$ ligand than bpy for ATRP reactions. Shen et al. (2004) studied different procedures for catalyst removal such as adsorption and extraction techniques, using supported catalysts with different temperature-dependent solubility. All these experiments were successfully conducted at laboratory-scale. However, implementation of the ATRP technique faces challenges in industry as it requires expensive reactants and metal-based catalyst with relatively high concentration. As a result, interest for more active catalysts with low concentration has been the focus of several studies which attempted to minimize the need for product purification (Matyjaszewski and Tsaversky, 2014; Krys and Matyjaszewski, 2017).

Innovative methods recently reported different strategies are focused in reducing the amount of $\mathrm{Cu}$ complex catalyst required in ATRP as synthesized polymers containing only tiny amounts of $\mathrm{Cu}$ are much safer and more feasible for industrial applications. In 2006, Matyjaszewski and coworker reported an ATRP variant called ARGET (activators regenerated by electron transfer), utilizing a non-toxic reducing agent such as ascorbic acid. In addition, the research group also reported another variant called ICAR (initiators for continuous activator regeneration), in which radical initiators are use. In both processes, the concentration of copper catalyst needed was reduced to 10-50ppm (i.e. several orders magnitude lower than in conventional ATRP). 


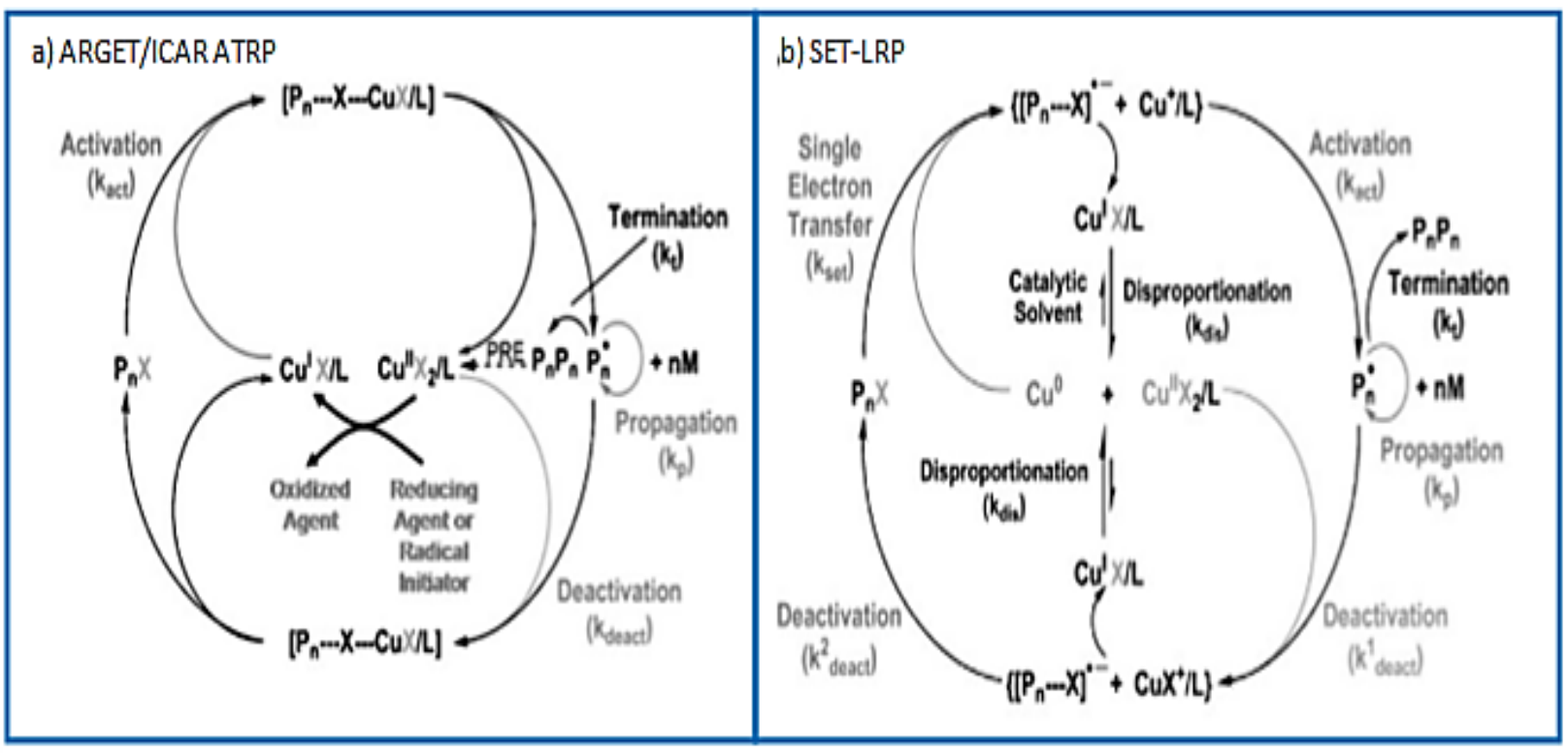

Figure 3.1: (a) ARGET/ICAR ATRP and (b) SET-LRP, two methods that strongly reduce the concentration of Cu catalyst in Cu-mediated CRP (Percec and Lligdas, 2007).

Percec and Lligadas (2007) is another group who contributed to the development of ATRP. Taking a different approach, they have converted ATRP into a more industrially friendly process by modifying its mode of action. In their technique called SET-LRP (Single-Electron Transfer Living Radical Polymerization), elemental copper $\left(\mathrm{Cu}^{0}\right)$ activates the polymerization and it is converted to a $\mathrm{Cu}^{\mathrm{I}}$ intermediate during the process (Figure 3.1). A spontaneous disproportionation reaction of the intermediate, which generates the $\mathrm{Cu}^{\mathrm{II}}$ deactivator is mediated by environmentally friendly solvents such as water or alcohols. The $\mathrm{Cu}^{0}$ catalyst used in this polymerization type is much more reactive than the $\mathrm{Cu}^{\mathrm{I}}$ species used in conventional ATRP, which implies that only small amount of elemental copper is needed (about $\sim 10 \mathrm{ppm}$ ). The $\mathrm{Cu}$ amount turned out to be close to the range in ARGET and ICAR. Besides, purification of the polymer product may not be necessary. In addition, SET-LRP process takes place at room temperature, which minimizes side reactions, reaction times are fast, and high molecular weight (> $106 \mathrm{~g} / \mathrm{mol}$ ) polymers are made. Lastly, both nano activated monomers like vinyl chloride and activated monomers like acrylates and methacrylates can be polymerized with low ppm amount of catalyst (Krys and Matyjaszewski, 2017). These recent developments have undoubtedly given a new dimension to $\mathrm{Cu}$-mediated CRP. 


\subsection{Various Components of the ATRP Mechanism}

\subsubsection{Initiator}

The role of the initiator is to provide an initiating radical species via the first activation/deactivation cycle of the polymerization. The number of initiators suitable for ATRP is much larger in comparison to other CRP methods. A variety of initiators (Figure 3.2) successfully employed in ATRP are organic halides with a carbon-halogen bond, which can easily generate a radical species through electronic and steric effects of their substituent. Halogens $(\mathrm{X})$ in the initiators (R-X) include chlorine, bromine, and iodine. Alkyl bromides are typically more active than chlorides due to the lower bond dissociation energy (BDE) of the $\mathrm{C}-\mathrm{Br}$ bond compare to $\mathrm{C}-\mathrm{Cl}$ bond (Tang et al., 2008). The C-I bond has even lower BDE, which imply much higher activity of alkyl iodides. However, the ATRP equilibrium constant ( $\mathrm{K}_{\mathrm{ATRP}}$ ) depends on the bonding of halide to the $\mathrm{Cu}$ species and the affinity of $\mathrm{I}$ towards $\mathrm{Cu}$ is much lower than that of $\mathrm{Br}$ or $\mathrm{Cl}$ (Krys and Matyjaszewski, 2017). Currently, chlorides and bromides have been widely employed.

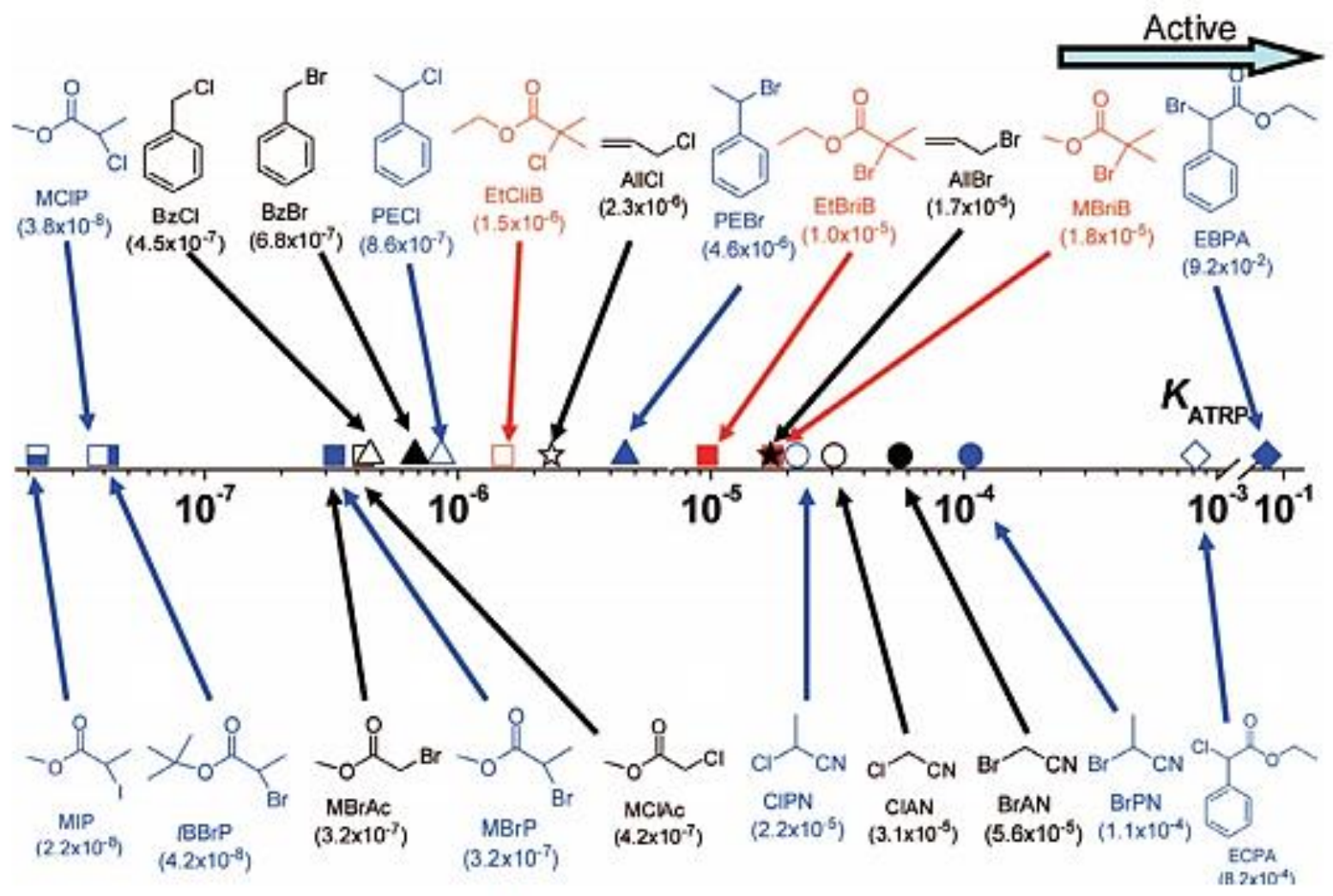

Figure 3.2: ATRP equilibrium constant for various initiators (Tang et al. 2008). 
In order to obtain a controlled polymerization, careful selection of initiators must be assessed in accordance with the structure and reactivity of the monomer and the metal complex (i.e. selection of 2-bromopropionitrile (BPN) for polymerization of acrylonitrile, methyl 2-bromopropionate (MBrP) for methyl acrylate, and 1-phenylethyl bromide (PEBr) for styrene) (Krys and Matyjaszewski, 2017). Furthermore, initiation must be quantitative, and the initiation step must be fast in comparison to propagation. The design of good initiators has been a challenging task for a better quality of the polymer products. However, due to the tolerance of CRP to functional groups, initiators are selected to introduce various functionalities into the polymer (Tang et al., 2008). For example, synthesised initiators that are disulfide-containing initiators, hydroxyl-containing initiators, alkyne-containing initiators, nucleoside-containing initiator some of the example are shown on Figure 3.3 (Van Camp et al., 2007)<smiles>COC(=O)C(C)Br</smiles>

methyl 2-bromopropionate

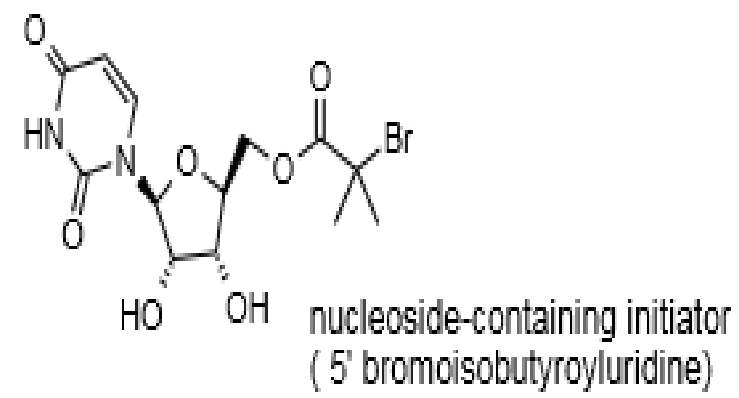<smiles>CC(C)(Br)C(=O)OCCCCO</smiles>

hydroxyl-containing initiator (also dual initiator for ATRP and CROP)

Figure 3.3: Example of initiator used in ATRP (Van Camp et al., 2007).

\subsubsection{Monomer}

A wide range of monomers can be successfully polymerized through ATRP: styrene, acrylates, methacrylates, dienes acrylonitiles, (meth)acrylamides (Van Camp et al., 2007). However, there 
are only a few reports on the attempted ATRP of acrylamides, such as N, N-dimethylacrylamide, but it was subsequently confirmed that these systems were not "living" (Rademacher et al., 2000). Currently, there are two major classes of monomers, which have not yet been successfully polymerized by ATRP. First, ATRP shows some restriction for polymerization of acid-containing monomers such as for the instance of (Meth) acrylic acid due to a competitive complexation of the monomer with the metal catalyst, which results to disruption of ATRP equilibrium. In addition, halogenated alkenes, alkyl-substituted olefins, and vinyl ester may be resistant to polymerization by ATRP (Krys and Matyjaszewski, 2017). Nevertheless, the range of monomers polymerizable by ATRP is greater than those polymerizable by nitroxide-mediated polymerization.

As observed by Haddleton and coworkers (1998), other additives like phenols (not good to environment) can accelerate the polymerization rate of methyl methacrylates, without compromising the control of the polymer molecular weight. In addition, carboxylic acids can enhance the polymerization rate, but the polydispersity increased with an increase in the acidcopper ratio.

\subsubsection{Ligand $(L)$}

Ligand is another important reactant for ATRP process. A significant number of studies have been conducted in order to understand the effect of ligands used with $\mathrm{Cu}$. It was found that the ligand structure (Figure 3.4) plays an important role on the activation rate coefficient with the following general trend: tetradentate $($ cyclic-bridged) $>$ tetradentate $($ branched) $>$ tetradentate $($ cyclic) $>$ tridentate $>$ tetradentate (linear) > bidentate ligands (Bergenudd, 2011). In addition, the nature of nitrogen atom, with pyridines being more active than the aliphatic amines and imines being the least active. Some of the commonly used amine ligands for $\mathrm{Cu}$-catalyzed ATRP are classified according to their number of nitrogen atoms as follows:

- Bidentate ligands (2N atoms): 2,2'-bypiridines or $\mathrm{N}$-alkyl-2-pyridine methane imines (bipy).

- Tridentate ligands (3N atoms): N, N,N',N",N"-pentamethyldiethylene-triamine (PMDETA).

- Quadridentate ligands (4N atoms): 1,1,4,7,10,10-hexamethyldiethylen-tetramine (HMTETA) or N, N,N',N',N",N"-hexamethyl(tris(2-aminoethyl)-amine (Me6 TREN). 


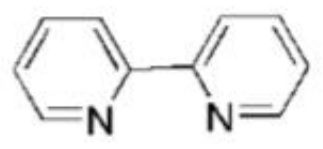

bipy

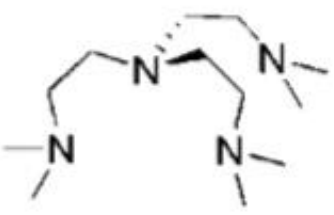

$\mathrm{Me}_{6}-\mathrm{TREN}$

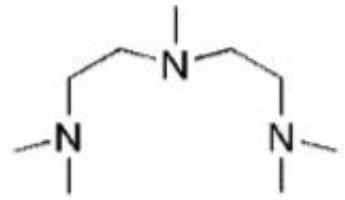

PMDETA

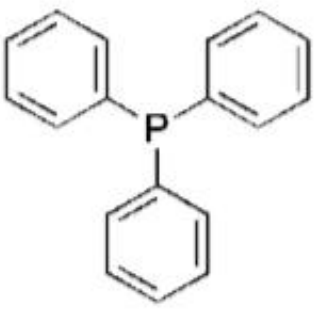

$\mathrm{PPh}_{3}$

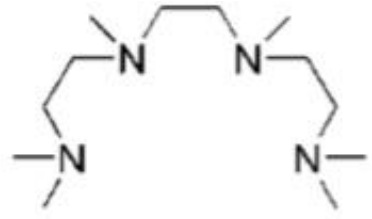

HMTETA<smiles>[R]N=Cc1ccccn1</smiles>

$\mathrm{N}$-(alkyl)-2-pyridylmethanimine

Figure 3.4: Common Ligand used in ATRP (Bergenudd 2011).

The main purpose of ligands in ATRP is to control the solubility of a transition metal (homogeneous reaction medium) in the reaction mixtures. In addition, ligands serve several purposes: they can tune the atom transfer equilibrium constants, adjust the selectivity of the catalysts, and ensure the stability of catalyst complex in different monomers, solvents, and temperatures (Qui et al., 2000). As a result, the solubility of the catalyst/ligand complex will help to determine the actual concentration of the catalyst in the reaction mixture, which affects the position of the reaction equilibrium. This in turn influences the overall kinetics of the polymerization as well as the MWD of the produced polymer.

\subsubsection{Transition Metal/ Additive in ATRP}

The effect of different transition metals on ATRP has been extensively studied. Currently, numerous transition metals such as $\mathrm{Cu}, \mathrm{Ru}, \mathrm{Ni}, \mathrm{Fe}$, and less frequently ones like $\mathrm{Re}, \mathrm{Pd}$, Mo have been utilized in ATRP. Transition metals play an integral role for a successful ATRP, as a small amount of a zero-valent metal, such as copper or iron can significantly enhance the polymerization rate (Van Camp et al., 2007; Krys and Matyjaszewski, 2017). 
For instance, $\mathrm{Cu}$ is the most studied and most used transition metal. Copper-based catalyst complex show a good reactivity. Several ligands are commercially available or easy to synthesize, and copper complex show a high selectivity for atom transfer (they possess a low affinity for alkyl radicals). However, due to the toxicity of the $\mathrm{Cu}$-catalyst and the intense colour of the resulting polymer, various post-polymerization purification methods have been attempted for the removal of the catalyst. As removal of the catalyst can be costly and time-consuming process, research concerning the development of a solid-phase catalyst system has been reported by several research groups.

\subsubsection{Solvent $(S)$}

ATRP can be carried out either in bulk, solution, or heterogeneous dispersed system (e.g. emulsion, suspension). The use of a solvent is necessary, especially in cases where polymers formed are not soluble in their monomers. Different solvents have been used for various monomers (i.e. benzene, toluene, xylene, diphenyl ether, ethyl acetate, DMF, ethylene carbonate, alcohol, and water). One of the key requirements for solvent selection is the absence of chain transfer to solvent. In addition, interaction between the solvent and the catalyst or other components in the ATRP systems are also important (Krys and Matyjaszewski, 2017). Catalyst poisoning caused by the solvent (i.e. carboxylic acids or phosphines in copper-based ATRP) and solvent-assisted side reactions (prominent in a polar solvent) should be minimal.

\subsection{Mechanism}

A typical ATRP system is considered as multicomponent system consisting of a monomer, a halogen initiator IX and a transition metal species in a lower oxidation state $\mathrm{Mt}^{\mathrm{n}}$ that is associated with a ligand $\mathrm{L}$. The transition metal complex $\mathrm{Mt}^{\mathrm{n}} / \mathrm{L}$ is responsible for the homolytic cleavage of the alkyl halogen bond in dormant chains which generates the corresponding higher oxidation state complex $\mathrm{Mt}^{\mathrm{n}+1} \mathrm{X} / \mathrm{L}$ and a primary radical $I^{\bullet}$ or polymer propagating radical $R_{r}^{\bullet}$. Then, the polymer propagating radicals reacts with monomer $\mathrm{M}$. The temporary radical $R_{r}^{\bullet}$ can either terminate in a dead polymer $\mathrm{P}_{\mathrm{r}}$ or $\mathrm{P}_{\mathrm{r}+\mathrm{s}}$, forms a dead $\mathrm{P}_{\mathrm{r}}$ by chain transfer or be reversibly deactivated by $\mathrm{Mt}^{\mathrm{n}+1} \mathrm{X} / \mathrm{L}$ to form a dormant halide polymer chain. Radical termination is diminished in ATRP as a result of the PRE, and the ATRP equilibrium $\left(\mathrm{K}_{\mathrm{ATRP}}=\mathrm{k}_{\mathrm{act}} / \mathrm{k}_{\text {deact }}\right)$ is strongly shifted towards the dormant 
species $\left(\mathrm{k}_{\mathrm{act}}<<\mathrm{k}_{\text {deact }}\right)$ (Matyjaszewski and Tsarevsky, 2014). The most common AGET ATRP kinetics mechanism is given in Table 3.1 .

Table 3.1: Most common AGET ATRP kinetics mechanism

Initiation

$$
\begin{aligned}
& M t^{n+1} / L+R A \rightarrow M t^{n} / L+O S \\
& I-X+M t^{n} / L+R A \stackrel{k_{\text {deact }} \stackrel{k_{\text {act }}}{\longrightarrow}}{\longleftarrow} M t^{n+1} X / L+I^{\bullet} \\
& I^{\bullet}+M \stackrel{k_{p}^{\prime}}{\rightarrow} R_{1}^{\cdot}
\end{aligned}
$$

Propagation.

$$
\text { Where } \mathrm{r} \geq 1 R_{r}^{\bullet}+M \stackrel{k_{p}}{\rightarrow} R_{r+1}^{\bullet}
$$

Termination

$$
\begin{aligned}
& R_{r}^{\cdot}+R_{s}^{\cdot k t c} \stackrel{\stackrel{k t}{\longrightarrow}}{r+s} \\
& R_{r}^{\cdot}+R_{\dot{s}} \stackrel{k t d}{\longrightarrow} P_{r}+P_{S}
\end{aligned}
$$

Chain transfer

$$
\begin{aligned}
& R_{\dot{r}}^{\bullet} H-T \stackrel{K_{t r}^{m o n}}{\longrightarrow} P_{r}+T^{\bullet} \\
& T^{\bullet}+M \stackrel{K_{1}^{m o n}}{\longrightarrow} R_{r}^{\bullet}
\end{aligned}
$$

Radical Equilibrium

$$
R_{r}-X+M t^{n} / L \stackrel{\stackrel{k_{\text {act }}}{\longrightarrow}}{\stackrel{k_{\text {deact }}}{\longrightarrow}} R_{r}^{\cdot}+X-M t^{n+1} / L
$$

Where: The variables used above defined as: $I X$ : Initiator, $I^{\bullet}$ : primary radical generated from the initiator, $M$ : monomer, $T-H$ : transfer agent, $R_{r}^{\bullet}:$ propagating radical of size $r$; where $r \geq 1, R_{s}^{\bullet}$ : propagating radical of size $s$; where $s \geq 1, P_{r+s} / P_{r} / P_{s}$ : dead polymers of length $r+s, r$ and $s$, respectively. Agent: refer to the different chemicals depending on the controlled radical polymerization initiation method used. $\mathrm{K}_{\mathrm{ATRP}}=\mathrm{k}_{\mathrm{act}} / \mathrm{k}_{\text {deact }}$ (Ayres, 2011; Braunecker and Matyjaszewski, 2007; Matyjaszewski and Tsarevsky, 2014; Nicolas et al., 2013). 
Fischer and Fukuda (2001) analyzed the PRE in NMRP and extended the analysis to ATRP. Their kinetic model shows that the concentration of the persistent radical $\left(\mathrm{Cu}^{\mathrm{II}}\right.$ complex for ATRP) and free radical concentration follow $\mathrm{t}^{1 / 3}$ and $\mathrm{t}^{-1 / 3}$, respectively. An important difference between NMRP and ATRP is that, the kinetics and chain control in ATRP depend not only on the persistent radical $\left(\mathrm{Mt}^{\mathrm{n}+1} \mathrm{X} / \mathrm{L}\right)$ ratio but also on the activator $\left(\mathrm{Mt}^{\mathrm{n}} / \mathrm{L}\right)$. Average molecular weight is estimated roughly by the ratio $\Delta[\mathrm{M}] /[\mathrm{RX}]_{0}$ and are not affected by the concentration of transition metal. Referring to the kinetics model below, the polymerization rate increases with initiator concentration and depends on the ratio of activator to deactivator concentration according to equation 3.3 (Ayres, 2011). Fischer (1999) developed the fundamental kinetic model below for the rate of polymerization and PDI of a CRP in homogeneous system:

$$
\begin{aligned}
& P D I=\frac{M_{w}}{M_{n}}=1+\frac{1}{M n}+\left(\frac{k_{p}\left([R X]_{0}-[R X]\right)}{k_{\text {deact }}\left[M t^{n-1} X / L\right]}\right)\left(\frac{2}{p}-1\right) \\
& R_{p}=k_{p}[M][P \bullet]=k_{p} K_{A T R P}[M][R X]_{0} \frac{\left[M t^{n} / L\right]}{\left[M t^{n-1} X / L\right]} \\
& {\left[M t^{n-1} X / L=\left(6 k_{i} K_{A T R P}^{2}[R X]_{0}^{2}\left[M t^{n} / L\right]_{0}^{2}\right)^{1 / 3} t^{1 / 3}\right]} \\
& {[P \bullet]=\left(\frac{K_{A T R P}[R X]\left[M t^{n} / L\right]}{6 k_{t}}\right)^{1 / 3} t^{-1 / 3}}
\end{aligned}
$$

Equation 3.1 shows that polydispersitiy becomes smaller with increasing monomer conversions, increasing deactivator concentration and decreasing $\mathrm{k}_{\mathrm{p}} / \mathrm{k}_{\text {deact }}$ ratio. One of the advantages of ATRP over NMRP is that a sub-stoichiometric amount of metal complex can be used as a catalyst. Catalyst with sufficiently high value of $\mathrm{k}_{\text {deact }}$ can therefore be used in lower concentration and still provide a control over PDI. On the other hand, too little catalyst may lead to uncontrolled polymerization or termination. In fact, the amount of catalyst lost due to termination reaction is equal to the amount of termination chains according to equation 3.5 (Krys and Matyjaszewski, 2017). As a result, the amount of catalyst needs to exceed the amount of terminated chains for the polymerization to progress.

$$
\Delta\left[M t^{n} / L\right]=\Delta\left[M t^{n+1} X / L\right]=\Delta\left[P_{\text {dead }}\right]=2 k_{I} \int[P \bullet]^{2} d t
$$




\subsection{CRP in aqueous dispersed media}

The most diverse method of carrying out radical polymerization is through aqueous dispersed media. Utilizing water as the dispersion medium is environmentally friendly compared to using volatile organic solvents. In addition, water allows excellent heat dissipation during polymerization. The low viscosity of the dispersed medium allows access to high weight fractions of polymer not readily accessible in solution or in bulk polymerizations. This compartmentalization effect results in a higher polymerization rate and a higher molecular weight in free radical emulsion polymerization than those are achievable in solution/bulk (El-Aasser and Lovell, 1998; Min et al., 2006).

For more than half a century, FRP has been done in aqueous dispersed media. Specifically, one type of aqueous dispersed media, emulsion polymerization, is the dominant industrial polymerization processes for preparation of directly usable stable colloidal latex (Qiu et al., 1999; Enright et al., 2005; Cunningham, 2003). Thus, research on ATRP has attempted to apply CRP in aqueous dispersed media in the past decade.

However, many challenges were encountered in attempting to conduct a CRP in an aqueous dispersed media. Combining the multi-component nature of CRP to the heterogeneous nature of an aqueous dispersed media was not simple. Some of the observed problems include decreased in colloidal stability, a wide particle size distribution, loss of control over polymerization, and low initiation efficiency (Maud et al., 2006). In addition, because CRP method has its own different unique features, a strategy that had successfully proven in bulk and solution CRP processes is not necessarily efficient for another CRP technique. As a result, to implement CRP in dispersed media requires a careful consideration of every aspect of the mechanism of each process.

In spite of all the challenges encountered, remarkable progress has been achieved for understanding CRP in aqueous dispersed media in the last two decades. To be specific, advances in understanding NMRP and RAFT systems have progressed primarily with respect for gaining better knowledge in the behavioural chemistry of the system in a multi-phase environment. On the other hand, ATRP have undergone a series of more significant variations in its reaction chemistry. In particular, understanding how the polymerization is initiated and how the process can be driven by its adaptation to aqueous dispersions. 


\subsection{ATRP in Industrial Perspective}

The development of ATRP in industrial application began through the ATRP consortium founded by Prof. K. Matyjaszewski at Carnegie Mellon University of Pittsburgh, PA (Destarac 2010). This project lasted for five years (1996-2000) and gave rise to several US patents issued and licenses signed with industrial partners. In 2001, CRP consortium was launched with main objective to assist its industrial members in developing materials for their markets based on CRP techniques.

In 2006, ATRP consortium formed the basis for Carnegie Mello University (CMU) Spin-off Company called ATRP Solutions that uses technology to develop novel materials. Since 2003, ATRP consortium has been licensed up to 8 of their 40 corporations funding the research at CMU (PPG Industries, Dionex, Ciba, Kaneka, Mitsubishi, WEP, ATRP Solutions and Encapson) (Destarar 2010). Licensees around the world have begun commercial production of specialty polymers. Among the active licensees, PPG Industries explored ATRP to design low polydispersity, functional polymer additives of various controlled architectures (including block, gradient, graft, star copolymers) for coating applications. ATRP products that were evaluated include adhesion promoters, pigment dispersants, crater control agents and flow additives for powder coatings (Destarac 2010).

According to Min and Matyjaszeswki (2006), ATRP process has been used commercially in the U.S., Europe, and Japan. Kaneka pioneered the use of ATRP process for large-scale polymer production. Polymers exhibit low polydispersity (1.1-1.3 for most grades) and high endfunctionality. A range of molecular weights and main chain compositions is available. In addition, these polymers offer excellent performances in weather ability, heat and oil resistance and have been developed for a variety of application such as sealants, adhesive, coatings, gaskets, and potting's (Destarac 2010).

D.M. Haddleton et al. (1998) from University of Warwick have actively participated in understanding and development of $\mathrm{Cu}$-mediated controlled radical polymerization. They founded Warwick Effect Polymers (WEP) in 2001, which focused in two main business areas: biopolymers and specialty polymers (Destaract, 2010). For biomedical applications, WEP focused on making PEGylation agents PolyPEG for conjugation of proteins, peptides, and other biomolecules. In addition, WEP had several collaborations with pharmaceutical and biotechnology companies, 
involving proteins and oligonucleotide therapeutics that have not yet matured into licenses and manufacturing contracts. Also, WEP developed GlycoPol, a potential drug targeting technology. The goal is to demonstrate the advantages over conventional PEGs for the development of a new generation of biological therapeutics (Destaract, 2010).

\subsection{ATRP of BMA in Emulsion Systems}

Polymerization of butyl methacrylate is investigated in this thesis. Polybutyl methacrylate (PBMA) was first produced in early 1930 by DuPoint and commercially sold as Lucite®45 (Epley, 1998)

Different grades of PBMA are widely used in both clear and pigmented coatings for plastics, sealers for concrete, high solids coatings and ink formulations, textile additives, paper coatings, fiber optics, exterior architectural and industrial paints etc.. It can copolymerize with methyl methacrylate to form resin which exhibits enhanced flexibility and toughness without the need for a plasticizer.

ATRP has been controversially the most popular CRP method and BMA is a very well-known monomer with versatile applicability as homopolymer or co-polymer. Therefore, there is extensive research interest of polymerizing BMA using ATRP technique. Only the most pertinent studies of PBMA in ATRP system are summarized next.

Peng et al. (2003) carried out reverse ATRP of n-butyl methacrylate in dispersed media. The authors carried out the experimental tests with different surfactants and Brij 35 gave the best result in terms of molecular control and colloidal stability. In addition, the authors carried experiments in three different ligands (dNbpy, bpy and bde) and concluded that dNbpy gave the best result.

Ibrahim et al. (2004) studied different factors affecting homogenous controlled polymerization of BMA in bulk and solution. The authors used $\mathrm{Fe}^{2+}$ metal as a catalyst complex and concluded that addition of small amount of $\mathrm{FeCl}_{3}$ as deactivator decreased the rate of polymerization and lowered the polydispersity of the final polymer product.

In another study, Peng and Cheng (2004) successfully carried out ATRP of n-butyl methacrylate in aqueous dispersed system using $50 \mathrm{ml}$ round bottom flask. The authors used a synthesized Ethyl 
2-bromopropinate as initiator and $\mathrm{CuCl}_{2} / \mathrm{dNbpy}$ as catalyst complex. The polymerization was well controlled, and the latex was stable.

Min et al. (2006) reported the development of a two-step ATRP emulsion of BA. In their study they tested FRP, normal ATRP, reverse ATRP, and AGET ATRP initiation techniques and claimed that AGET ATRP was the best in term of overall control of the polymerization and stability of the latex.

Wei et al. (2014) reported the single step emulsion AGET ATRP of BMA using $\mathrm{CuCl}_{2} / \mathrm{dNbpy}$, VA-044 as initiator, EBiB, ascorbic acid, SL (surfactant ligand) and Tween 80, followed by chain extension with MMA in solution ATRP. The authors reported uncontrolled polymerization when using water soluble initiator VA-044. However, using oil soluble initiator EBiB, they reported making a polymer with a relatively broad PDI $(1.69,1.56$ and 1.49$)$ based on the amount of dNbpy in the system.

Upadhayay et al. (2017) reported the single- and two-step procedure of AGET emulsion ATRP of methyl methacrylate. In their study, they successfully carried out AGET ATRP of methyl methacrylate in a $2 \mathrm{~L}$ reactor batch reactor. They concluded that with improved latex stability, the two-step emulsion AGET ATRP is a promising polymerization technique to produce well controlled and structured polymer.

To our best knowledge to date, there is no published paper that synthesized PBMA in two-step emulsion AGET ATRP.

\subsection{Project Goal}

CRP techniques in aqueous dispersed media is progressing rapidly because of continuous regulatory pressure requiring polymerizations in an industrially viable and environmentally friendly processes. The major objective of this thesis is to explore the feasibility of conducting AGET ATRP of BMA in emulsion. The study is to collect real data of PBMA in AGET ATRP for the understanding of multiphase system. This required development of understanding the role that each reaction component plays for achieving the best experimental scenarios for the synthesis of well-defined PBMA polymer in an ATRP process. 


\section{CHAPTER 4}

\section{EXPERIMENTAL PROCEDURE AND CHARACTERIZATION TECHNIQUES}

\subsection{Reagent Purification}

The monomer (Butyl Methacrylate, Sigma Aldrich) was purified by passing it in a column to remove the inhibitor. The column was purchased from Sigma Aldrich (Canada). The purified monomer was placed in a dark brown reagent bottle and stored in fridge at $2-6{ }^{\circ} \mathrm{C}$. The solvents methanol and THF (tetrahydrofuran) and other components (i.e. dNbpy, Ebib, AA) were used as received from the suppliers (Sigma-Aldrich and Fischer Scientific) without further purification.

\subsection{Experimental Set-up}

Polymerization runs were performed in a $2 \mathrm{~L}$ stainless steel PARR reactor vessel (series 4530) that is connected to a temperature controller and heating/cooling bath (Cole-Parmer Polystat H28L). A detailed schematic diagram provided in Figure 4.1. The reactor system is equipped with temperature (series 4848) and an impeller speed controller unit.

The reactor vessel is a flat-bottomed cylindrical tank with a diameter of $10.16 \mathrm{~cm}$ and a height $26.67 \mathrm{~cm}$. The reactor vessel is equipped with a $45^{\circ}$ pitched-blade turbine of a $5 \mathrm{~cm}$ diameter. The power of the stirring motor is $1 / 4 \mathrm{hp}$. In addition, a $\mathrm{U}$-shaped cooling coil connected to the circulator bath (Cole-Parmer Polystat H28L). The reactor temperature was measured online by means of a type $\mathrm{J}$ thermocouple which transmits the signal to a computer. The temperature history was recorded during each experimental trial. Single pipe for inlet of reactants or outlet of solution sampling and gas purging pipe were mounted in the reactor vessel. Detailed description of the $2 \mathrm{~L}$ reactor in provided in a previous thesis (Roudsari, 2015).

The reactor was run with distilled water for few hours prior to the beginning of actual experimental trials to ensure removal of any residue left from previous experiments. During this period, the reactor tuning was performed, where assessment of the control of the heater and reactor temperature, motor speed and reactor pressure was achieved. 


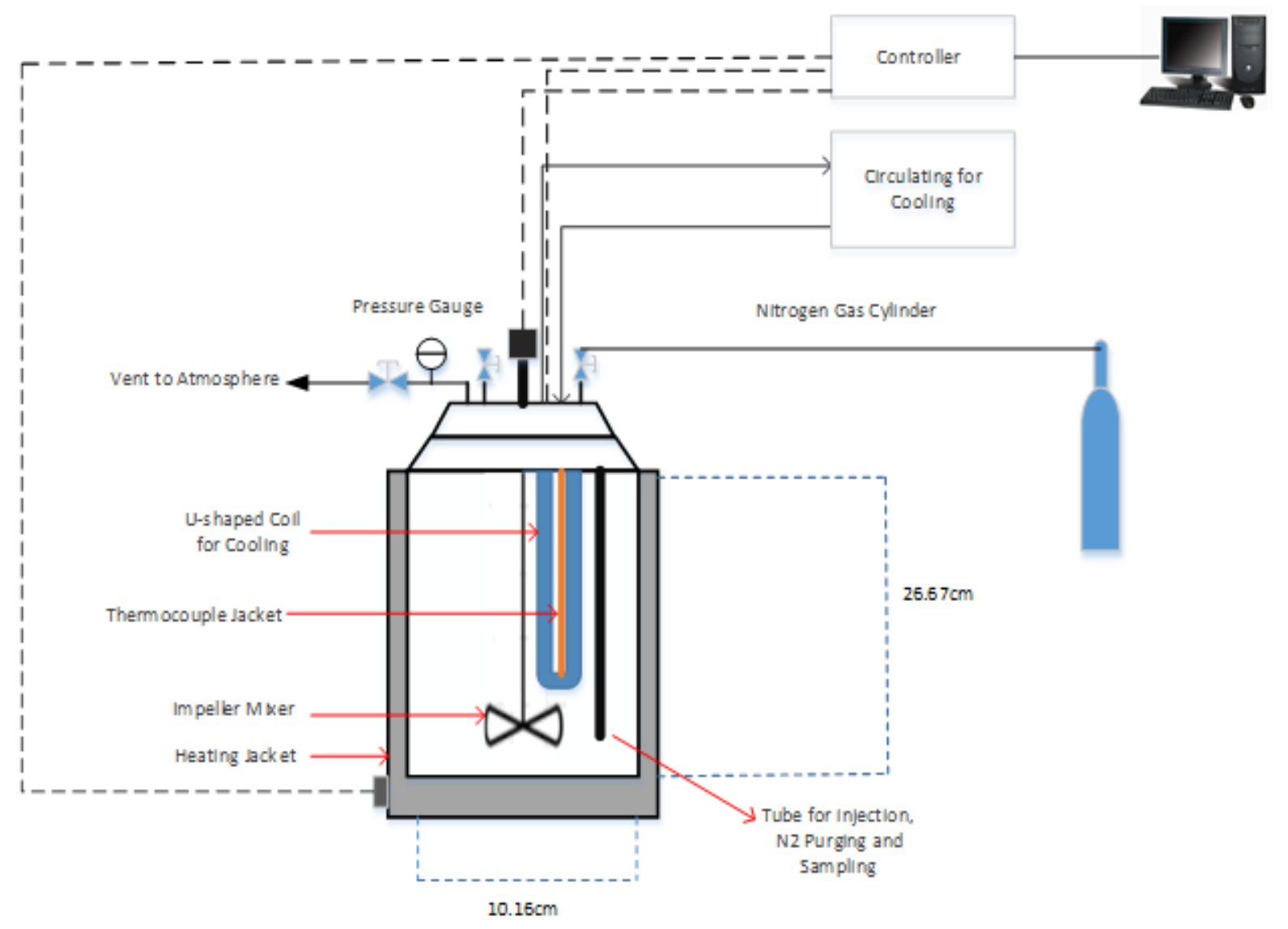

Figure 4.1: 2L Stainless Steel Reactor System.

\subsection{Polymer Characterization}

\subsubsection{Conversion}

In the present study, off-line gravimetry was used to determine the monomer conversion of each collected sample. An aliquot of each sample was weighed into an aluminum cup, dried in a vacuum oven kept at $45-50^{\circ} \mathrm{C}$ for minimum of $24 \mathrm{~h}$ where water, unreacted monomer and any volatile 
species were evaporated. Thus, the remaining solid is mainly poly-BMA and some negligible traces of other solids (catalyst complex, surfactant, initiator, and reducing agent).

From the formulation of the reaction, the mass fraction of each component is calculated. Knowing the initial amount of monomer and other reactants; the mass of monomer converted is equal to that of the polymer collected in the dried sample, and considering the principle of mass conversation, conversion can be calculated.

The weight fraction of the solid is provided by Penlidis (1986):

$$
\text { w. } f \text { of solids }=\frac{(\text { weight of cup }+ \text { dry residue })-(\text { weight of cup })}{(\text { weight of cup }+ \text { wet sample })-(\text { weight of cup })}
$$

The weight of each cup is measured before putting the wet sample therein. The weight fraction of each component is known from the employed formulation of the reaction. Therefore, the conversion is (Penlidis, 1986):

$$
x(t)=\frac{w \cdot f \cdot s o l i d s-(w \cdot f \cdot \text { initiator }+w \cdot f \cdot c a t a l y s t \text { complex }+w \cdot f \cdot s u r f a c t a n t+w \cdot f \text { reducing agent })}{\text { w.f.monomer }}
$$

\subsubsection{Gel Permeation Chromatography (GPC)}

Size exclusion chromatography (SEC) also referred to as Gel Permeation Chromatography (GPC), is the most popular and convenient characterization technique for determining the average molecular weights and molecular weight distribution of a polymer. As its name implies, SEC works on the principle of separating of the polymer chains according to their size. In GPC, a very low concentration $(1-5 \mathrm{mg} / \mathrm{ml})$ of polymer solution is passed through a column of porous particles (Striegel et al., 2009). The molecules of large polymer particles cannot enter the pores of the packing, and thus they elute faster. On the other hand, smaller polymer molecules that can penetrate and diffuse through the pores are retained inside the column for a while and then elute later. As a result, samples are fractioned by molecular hydrodynamic volume and the resulting profile describes the molecular weight distribution. A concentration detector (i.e. differential refractometer) is placed downstream of the column to measure the concentration of the polymer for each fraction as a function of time. 
In this study, measurement of the molecular weights of the collected polymer samples were performed using GPC (Viscotek TDA, Model 302) equipped with triple detector array in which tetrahydrofuran was used as the mobile phase at a nominal flow rate of $1.0 \mathrm{~mL} / \mathrm{min}$. The characteristic system is equipped with an integrated pump, Viscotek GPC Max VE 2001 GPC solvent/sample module as auto sampler, a degasser followed by Viscotek's triple detector equipped with a low- and right- angle laser light scattering detector (LALLS/ RALLS), differential refractor (RI) and a viscometer in series. The mobile phase, stored in solvent bottles placed on top of the equipment, enters the dual piston pump after passing through the eluent sensor. The inline degasser then removes dissolved gases from the mobile phase to improve the detection baseline.

\subsubsection{Quantification and Molar Mass Distribution}

Based on the elution volume, GPC is commonly used to characterize the distribution of polymers. The shape of the elution curve can describe the presence of one or more populations and the variation in polymer molecular weight for a population.

\subsubsection{Number Average Molecular Weight}

The number average molecular weights $\left(\mathrm{M}_{\mathrm{n}}\right)$ is the conventional and traditional average molecular weight of the distribution of polymers based on the number of chains in a sample. The GPC computer software calculates $\mathrm{M}_{\mathrm{n}}$ for an elution peak automatically by considering the intensity of the refractive index detector response at each observed elution volume.

$$
M_{n}=\frac{\sum N_{i} M_{i}}{\sum N_{i}}=\frac{\sum_{i=1}^{N} h_{i}}{\sum_{i=1}^{N}\left(h_{i} / M_{i}\right)}
$$

Equation 4.3 (Striegel et al., 2009) shows that $\mathrm{M}_{\mathrm{n}}$ (units of $\mathrm{Da}$ or $\mathrm{kDa}$ ) is calculated by obtaining the total weight of all polymers by summing up all the number of polymer chains $\left(\mathrm{N}_{\mathrm{i}}\right)$ times the molar mass of the polymer chain $\left(\mathrm{M}_{\mathrm{i}}\right)$ for a particular length $\mathrm{i}$ and divided by the total number of polymer $\left(\sum N_{i}\right)$. The elution peak curve is actually used to calculate the average number of molecular weight $\mathrm{M}_{\mathrm{n}}$. The measurement is done by recording the refractive index signal intensity $\mathrm{h}_{\mathrm{i}}$ for the molar mass $\mathrm{M}_{\mathrm{i}}$ corresponding to each i-th elution volume.

\subsubsection{Weight Average Molecular Weight}

The weight average molecular weights $\mathrm{M}_{\mathrm{w}}$ of each sample is a commonly reported variable in the characterization of polymers samples. The $\mathrm{M}_{\mathrm{w}}$ is a weight which is more related to higher 
molecular weight polymers and it therefore provides a more accurate representation of the polymer average molecular weights. The GPC software calculates $\mathrm{M}_{\mathrm{w}}$ by considering the refractive index signal intensity and the corresponding molar mass for each i-th interval in the elution curve, as follows (Striegel et al., 2009):

$$
M_{w}=\frac{\sum N_{i} M_{i}^{2}}{\sum N_{i} M_{i}}=\frac{\sum W_{i} M_{i}}{\sum W_{i}}=\frac{\sum_{i=1}^{N} h_{i} M_{i}}{\sum_{i=1}^{N} h_{i}}
$$

\subsubsection{Polydispersity Index PDI}

The polydispersity index PDI is a measure of the dispersion of the polymer chains distribution and it can provide information about the uniformity of the polymer chains as well as any presence of branching. PDI is defined as the ratio of $\mathrm{Mw}$ over $\mathrm{M}_{\mathrm{n}}$ and it is given below:

$$
P D I=\frac{M_{w}}{M_{n}}
$$

Hence, a monodisperse polymer has a PDI value close to unity. However, no GPC experiment can ever generate a PDI of equal to unity, as the elution curves generated by analyzing monodispersed polymers have finite widths due to uncertainty and chromatographic effects. Polymer standards have therefore a PDI slightly higher than unity when analyzed by means of GPC.

\subsubsection{Band Broadening}

Band broadening (BB) occur when the detected signals widens due to chromatographic process and other column effects. Since BB is an inherent effect of the chromatographic process, it is not possible to eliminate it in a GPC test. However, BB can be reduced through adjustment of the chromatographic method and instrumentation set-up. Three inherent processes in chromatography can cause band broadening (Striegel et al., 2009):

- The mobile phase transports the analyte through the column. The analytes elute through the column and encounter multiple routes to follow the different flow speeds and lengths. As a result, the analytes spread within the column and the peak width may increase.

- The solvent molecules comprising the mobile phase are constantly migrating in and out of the stationary phase pores during a GPC test. However, the solvent molecules travel slowly through the column since the flowrate inside the pore is much slower than the flowrate outside the pores. The analytes that elute through the column are subject to differing mobile 
phase flowrates. As a result, the analytes may spread out within the column due to nonuniform flow rates. This phenomenon is called the mobile phase mass transfer and considerable BB process may occur in GPC.

- Stationary phase mass transfer occurs when the analyte dispersion is caused by nonuniform diffusion into the pores of the stationary phase.

\subsubsection{Dynamic Light Scattering (DLS)}

The DynaPro Plate Reader III (Wyatt Technology) was also utilized to measure polymer particle size by using Dynamic Light Scattering (DLS) with size range of 0.5 to $1000 \mathrm{~nm}$ and setting-up the detector position at $173^{\circ}$. Samples were prepared and diluted prior to analysis. The light intensity analysis displays the results based on intensity particle size distribution. Large particle scatter much more light than small particles, and the intensity of light scattering of a particle is proportional to the sixth power of its diameter according to Rayleigh approximation (WYATT, 2014).

\subsection{Reliability of Measurement}

There are several sources of error in an experimental work. Experimental errors may result from measurement inaccuracy, preparation of solutions and equipment accuracy. In this experimental work, precaution was taken to lower the level of error.

\subsubsection{Scale}

A scale (Meter Toledo AB 104-S) of high sensitivity was used to weigh chemical samples with high precision measurement. Calibration was done by adjusting the measurement of an internal weight. In addition, the Toledo AB-S certified models have an internal weight that adjusts itself automatically twice within $2 \mathrm{~h}$ of connection to the power supply.

\subsubsection{Controller Tuning}

At the reactor system level, a proper control over the temperature and impeller speed had to be intermittently tuned within the reactor controller system (PARR series 4848). This was generally done twice a year following the automatic tuning procedure specified by the manufacturer PARR. 
The procedure involved filling the reactor with distilled water to the volume to be used during the actual reaction $(\sim 500 \mathrm{ml})$. The reactor and its accompanying accessories were then tuned while mimicking the reaction parameter conditions. Then, the controller was turned on and the autotune setting was selected from the panel. The controller should bring the process to the desired temperature setpoint, while disturbing the system until it stabilized. This procedure is done by adjusting the PID controller parameters that can work reasonably well.

\subsubsection{Leak Testing}

A periodic leak test was done on the reactor system to ensure that the experimental setup remained free of leaks prior to using the reactor vessel. Also, one should make sure that the integrity of the closed system is able to minimize the amount of nitrogen needed for the duration of the polymerization reaction. In addition, the test is to remove any unnecessary residue left within the system that may dissolve in the distilled water.

This test involved filling the reactor to $80 \%$ of its volume with distilled water, sealing it and placing it under a blanket of nitrogen at high pressure of 20psig. The test began with preparing a soap/water solution in a separate container. The soap/water solution was then sprayed over the crucial tie-in connections of the closed reactor system to detect any leakage. If any soap bubble appeared, sealing adjustment onto the reactor was then verified until the disappearance of any leakage.

\subsubsection{GPC Calibration}

A set of relatively monodispersed polymers is usually used as a polymer standard. For GPC calibration, commercially available polystyrene (PS) standards were utilized. The peak elution volume was used to mark the elution volume for the molecular weight of the polymer. Polystyrene standards were sequentially injected into the column and their peak elution volumes were recorded and measured against the measured molecular weights of the eluted samples.

\subsubsection{Chromatogram Characteristics}

A typical GPC elution chromatogram is shown in Figure 4.2. Prior to analysis, the GPC instrument should be conditioned so that the detector response remains constant. The pertinent peak of interest lies within the calibration curve, which represents the polymer being analyzed. Other peaks in the 
chromatogram include the air peak, arise from dissolves gases in the polymer solution sample, or artefact/erroneous peak.

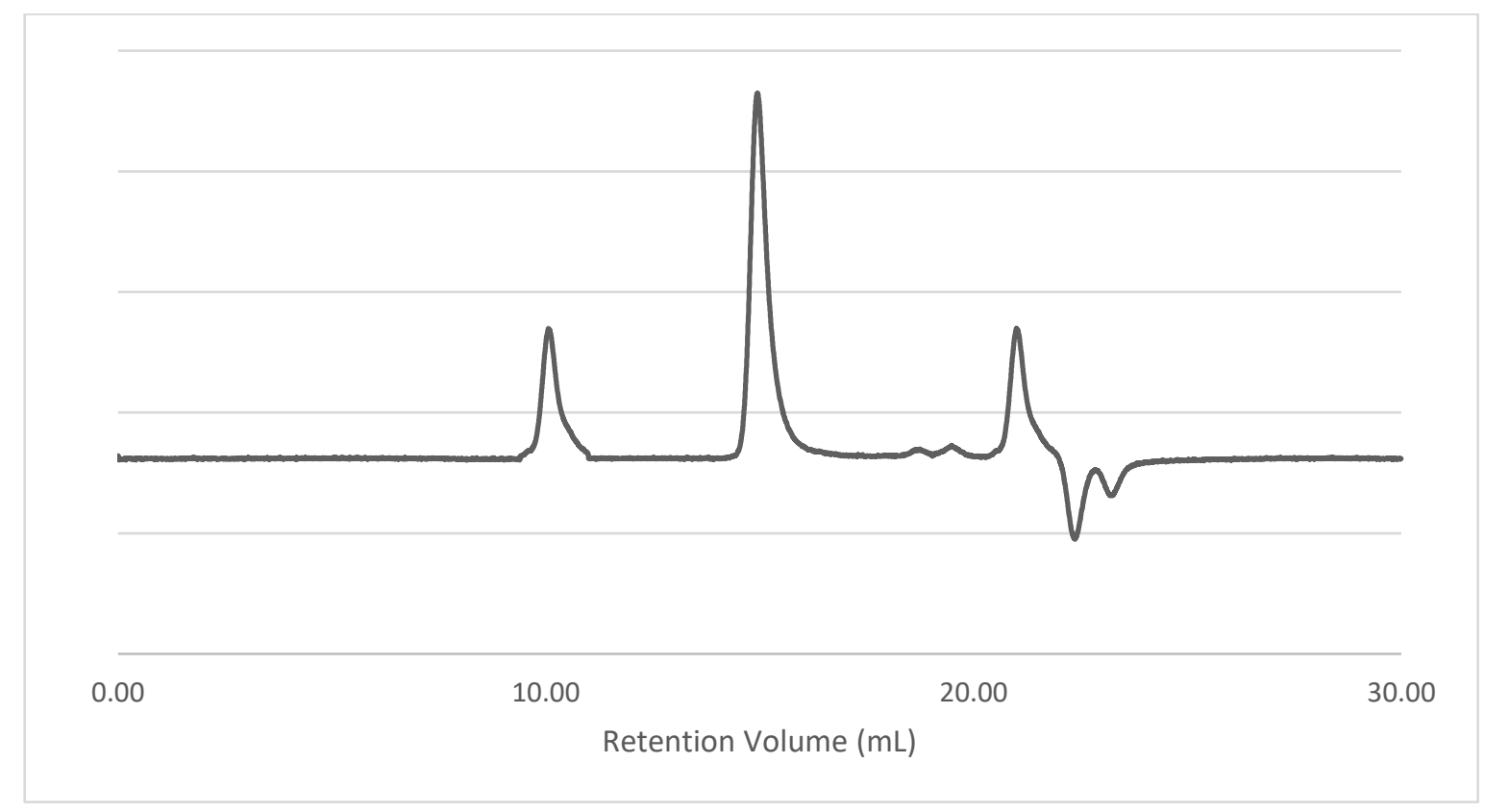

Figure 4.2: GPC Calibration Curve.

During the elution of the polymer samples, the detector measures the refractive index of the mobile phase after eluting through the column. The analyte must be detectable by the refractive index, even though the analyte should ideally be influenced by the sample concentration and not through the molar mass of the sample. Normally, plot in Figure 4.2 shows the GPC results produced multiple peaks. From the point of injection onwards, the refractive index signal is defected and recorded as a function of the elution volume. The peak of interest is the analyte peak, which should fall within the elution volume range of the polymer standards, i.e. Polystyrene. An air peak near the tail-end of the chromatography indicates residues of air in the mobile phase. In addition, an artefact from a previous GPC run is present, which could be due to contaminants in the fittings, pumps or columns. The artefacts are not observed if the sample is the first of the series of analyses but may appear in subsequent runs. 


\section{CHAPTER 5}

\section{PRELIMINARY INVESTIGATION OF AGET ATRP OF BMA IN EMULSION POLYMERIZATION}

\subsection{Introduction}

One way of assessing the possibility of a successful ATRP in a conventional emulsion procedure is to identify if the monomer and catalyst can effectively diffuse from monomer droplet across the aqueous phase to micelles or polymerizing particles. If the droplets cannot be transported, the emulsion ATRP may behave as a suspension polymerization. The requirement for the diffusion of monomer from monomer droplets to the polymerizing particles was demonstrated in a free radical emulsion polymerization (Harkins, 1947). It was demonstrated that as the monomer is consumed in the polymerizing particles, there is a continuous influx of monomer droplets into the growing polymer particles (Min et al., 2005). Further evidence for the monomer diffusion comes from semibatch emulsion polymerization procedures, in which, a fraction of the total monomer content is introduced at the beginning of the reaction prior to nucleation. The remainder of the monomer is

subsequently added according to a predetermined schedule throughout the polymerization. During the semi-batch emulsion process, monomer constantly transfers across the aqueous phase to polymer particles, as long as the polymerization loci remain inside the polymerizing particles (Min et al., 2005; Upadhayay, 2016).

However, even though the monomer diffusion is possible, transportation of catalyst from monomer droplet to the polymerizing particles remains a major challenge for a standard emulsion ATRP system. Another challenge encountered in ATRP is the catalysts decomposition, which is still unknown. The catalyst may decompose either through ligand decomplexation or through hydrolysis of $\mathrm{Cu}-\mathrm{X}$ bond (Nishikawa et al., 1997; Min et al., 2005; Oh, 2008). The decomposition of the catalyst can be prevented using hydrophobic ligands when conducting ATRP in aqueous dispersed media. As a result, the catalyst/ligand complex is assumed to remain within the monomer droplets and therefore does not transport through the aqueous phase to the polymerizing particles. For the reason stated above, a conventional emulsion polymerization is unlikely to be a viable 
technique for the ATRP process since only the monomer particles can efficiently diffuse from monomer droplets to the polymerizing particles.

Therefore, in order to overcome this obstacle for emulsion ATRP system, it was necessary to develop a procedure so that the catalyst components remain close to the monomer nuclei before the initiation of polymerization starts. Subsequently, the monomer can be added once the nucleation period is completed. This phase of newly developed microemulsion ATRP can be matched with the nucleation step for a regular emulsion polymerization procedure. ATRP microemulsion leads to a micro latex, which acts as the polymerizing particles to initiate an emulsion polymerization. The microemulsion latex is formed without using a high shear and therefore possibly scalable to industrial viable systems (Upadhayay, 2016). Lastly, since the catalyst/ligand complex has been captured in the initially formed micro latex, the polymerization should in principle occur exclusively inside the micro latex (i.e. polymerizing particles).

\subsection{Description of Experimental Procedure}

\subsubsection{Materials}

In this study, AGET ATRP was carried out in a 2L stainless steel stirred tank reactor and a detailed reaction procedure is provided in chapter 4 . The reaction recipe is given below in Table 5.1 and it consists of monomer, initiator, catalyst/ligand complex, surfactant, a reducing agent (ascorbic acid) and distilled water as the inert medium. The monomer n-Butyl methacrylate (BMA, Aldrich) was purified by passing through a column packed with inhibitor remover (Aldrich). The other compounds copper (II) bromide (Aldrich), Brij98 (Aldrich), ethyl 2-Bromoisobutyrate (Aldric), L-Ascorbic Acid (Sigma-Aldrich) were used as received.

The reducing agent was employed to limit the use of the copper catalyst which is not desirable for environmental reasons. The reducing agent is assumed to react with the catalyst only to enforce the transition metal complex low oxidation state which helps to generate initiator primary radicals as shown in the kinetic mechanism in Table 3.1. 
Table 5.1: Reaction Materials.

\begin{tabular}{|c|c|c|c|c|}
\hline \multirow[t]{2}{*}{ Reactants } & \multicolumn{4}{|c|}{ Chemical Component } \\
\hline & $\begin{array}{l}\text { Molecular } \\
\text { Weight } \\
(\mathrm{g} / \mathrm{mol})\end{array}$ & Acronym & Full Name & Purity \\
\hline Monomer & 142.20 & BMA & Butyl Methacrylate & $\begin{array}{l}99 \%(<30 \mathrm{ppm} \\
\text { MEHQ as } \\
\text { inhibitor })\end{array}$ \\
\hline Initiator & 195.05 & EBib & Ethyl-2-bromoisobutyrate & $>98 \%$ \\
\hline Catalyst & 223.37 & $\mathrm{CuBr}_{2}$ & Copper (II) Bromide & $99 \%$ \\
\hline \multirow[t]{3}{*}{ Ligand } & 408.66 & dNbpy & $\begin{array}{l}\text { 4-4'-Dinonyl-2,2'- } \\
\text { dipyridyl }\end{array}$ & $97 \%$ \\
\hline & 173.30 & PMDETA & $\begin{array}{l}\mathrm{N}, \mathrm{N}, \mathrm{N}^{\prime}, \mathrm{N} ", \mathrm{~N} "- \\
\text { pentamethyldiethylene- } \\
\text { triamine }\end{array}$ & $99 \%$ \\
\hline & 140.19 & HMTA & $\begin{array}{l}1,1,4,7,10,10- \\
\text { hexamethyldithylen- } \\
\text { tetramine }\end{array}$ & $99 \%$ \\
\hline Surfactant & 1149.53 & Brij 98 & $\begin{array}{l}\text { Polyoxyethylene (20) } \\
\text { oleyl ether }\end{array}$ & Not determined \\
\hline $\begin{array}{l}\text { Reducing } \\
\text { Agent }\end{array}$ & 176.12 & AA & Ascorbic Acid & $99 \%$ \\
\hline $\begin{array}{l}\text { Dispersion } \\
\text { Medium }\end{array}$ & 18.02 & Water & Water & Distilled Water \\
\hline $\begin{array}{l}\text { Polymer } \\
\text { precipitant }\end{array}$ & 32.04 & Methanol & Methanol & $99.80 \%$ \\
\hline GPC Solvent & 72.11 & THF & Tetrahydrofuran & $\begin{array}{l}>99 \% \text { HPLC } \\
\text { Grade }\end{array}$ \\
\hline
\end{tabular}

\subsubsection{Two Step-Emulsion Procedure}

A series of PBMA latexes were synthesized using the two-step emulsion polymerization technique (Upadhayay, 2016). The experimental runs and the reagents amounts are provided in Table 5.3. In the two step AGET ATRP polymerization in emulsion medium, catalyst $\left(\mathrm{CuBr}_{2}\right)$ and ligand (dNbpy) were first dissolved in BMA 1 (about $1 / 3$ of the total BMA amount) to form the organic phase (catalyst complex). Once the catalyst complex mixture was prepared, the initiator (EBiB) was then added. In the meantime, another aqueous solution composed of surfactant (Brij 98) and 
dispersion medium (Distilled water) was prepared, and a solution of reducing agent (ascorbic acid, g) in $30 \mathrm{ml}$ of water.

Once the whole solution was completely dissolved, the organic butyl methacrylate (BMA I) solution containing the catalyst complex and initiator was slowly poured into the aqueous solution of Brij 98 at $50^{\circ} \mathrm{C}$, while stirring to form an optically translucent and transparent microemulsion as depicted in Figure 5.1. The microemulsion was then transferred into the reactor which content was stirred at $250 \mathrm{rpm}$.

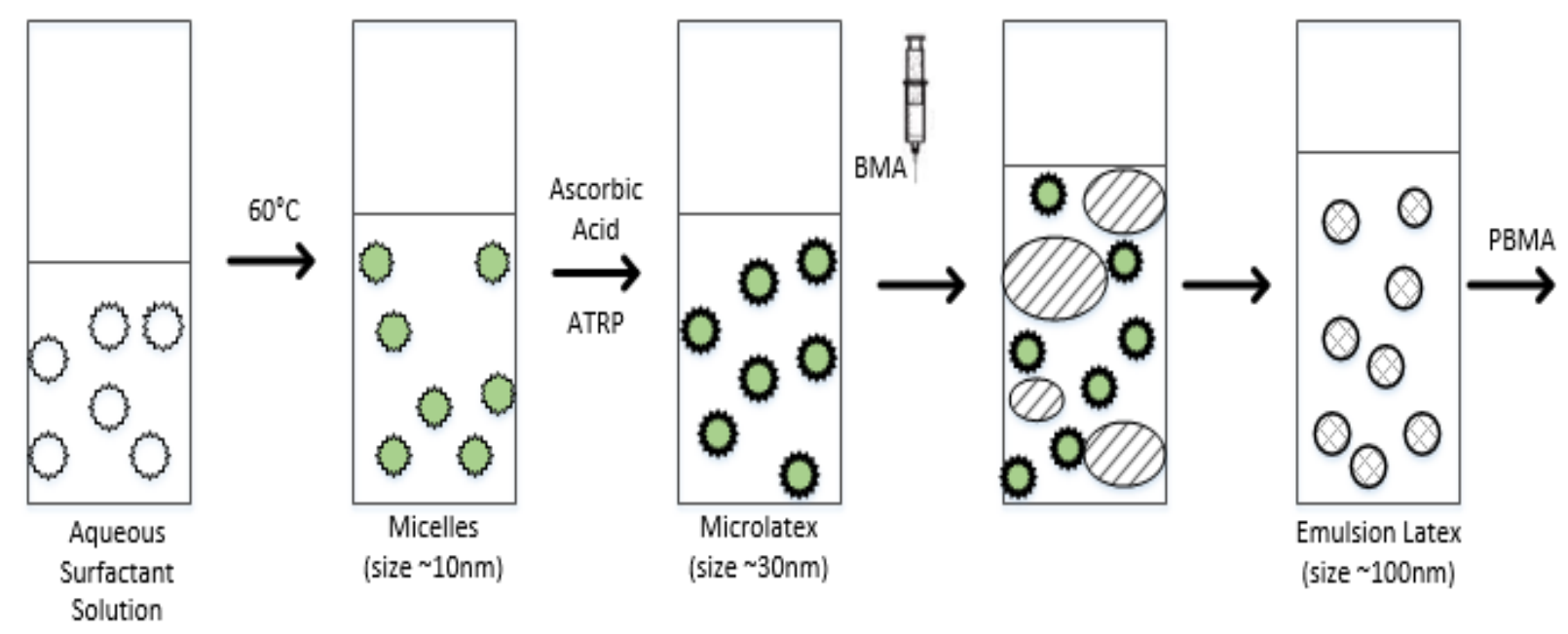

Figure 5.1: Synthesis of two-step emulsion polymerization of BMA in AGET ATRP.

The reactor was purged vigorously with nitrogen gas for about 5 minutes to limit the amount of air in the system (Massicotte, 2016). The reactor content was heated by the electric plate mounted inside the reactor vessel. Once the target temperature of $70^{\circ} \mathrm{C}$ was reached, the solution of the reducing agent (ascorbic acid, 10ml) was injected into the reactor to activate the catalyst/initiator mixture and initiate the polymerization. After 12 mins of initiation reaction, the second portion of butyl methacrylate (BMA II) was added to the ongoing microemulsion polymerization to form an emulsion polymerization. Then, the second portion of ascorbic acid solution (10ml) was immediately injected. Polymerization was carried out under an inert blanket of nitrogen gas. Polymer samples were withdrawn at selected times. The samples were kept in the fridge for later 
use. Each aliquot sample was shaken to mix it with any air trapped inside the vial and then cooled to stop the polymerization.

\subsubsection{Characterization}

Prior to sample analysis, polymer samples were dried in an oven at $50^{\circ} \mathrm{C}$ overnight and calculated accordingly to the procedure discussed in section 4.3.1. Monomer conversion was determined gravimetrically. Molecular weight and polydispersity of the polymer samples were determined by means of Gel permeation chromatography (GPC). Dried polymer samples were dissolved in tetrahydrofuran (THF) for molecular weight analysis using GPC, which is connected to Viscotek TDA (Model 302) equipped with auto sample, HPLC pump at 1.0mL/min and the Viscotek's triple detector. The triple detector is equipped with low- and right-angle viscometers in series.

\subsection{Preliminary Investigation of Two-Step Emulsion Procedure}

\subsubsection{Ligand Selection}

An important key for controlling the radical polymerization is to maintain a rapid equilibrium between the growing radicals and the dormant species. In ATRP, the presence of an $\mathrm{M}_{\mathrm{t}}^{\mathrm{n}+1} /$ Ligand complex is necessary to reduce the concentration of the radical through the deactivation process and maintain the activation-deactivation equilibrium for the controlled growth of the polymer chains. Ligands strongly affects equilibrium reaction in ATRP. For a specific ligand, the concentration of propagating radicals and the rate of deactivation must be adjusted to maintain polymerization livingness in ATRP. To obtain a well-defined polymer with low polydispersities, it is crucial to rapidly deactivate the growing chains to form dormant species. Thus, the selection of a suitable ligand is essential to obtain a well-controlled ATRP process. Copper $\left(\mathrm{Cu}^{\mathrm{II}}\right)$ is hydrophilic and tend to leave the organic phase. This tendency is countered by a hydrophobic ligand which forces the $\mathrm{Cu}$ (II) to remain within the polymerizing phase.

For the ATRP aqueous polymerization, it is critical to keep the catalyst/ligand complex attached to the monomer of the emulsion mixture. As foresaid, this is usually accomplished with the used of hydrophobic ligand. For instances, the most used hydrophobic ligand is Bis (2pyridylmethyl)octadecylamine (BPMODA). Unfortunately, this ligand is not commercially 
available. Therefore, in the preliminary step of this study we have investigated the effects of three ligands (PMDETA, HMTA, dNbpy) that are commercially available. The first ligand (PMDETA) is hydrophilic, the second one (HMTA) is moderately hydrophobic and the third ligand (dNbpy) is strongly hydrophobic. The investigation was conducted using similar molar ratio for each ligand systems and are summarized in Table 5.2. For all experimental runs, polymerization was carried out under 20psig pressure of nitrogen gas, a temperature of $50^{\circ} \mathrm{C}$, and a stirring rate of 250rpm. BMA 2 was added after $12 \mathrm{~min}$ of initiation. Nitrogen purging was done during the first 5min. The initial amount of AA was divided into two equal halves. The second half of AA (dissolved in $10 \mathrm{ml}$ of distilled water) was added immediately after the addition of BMA 2.

Table 5.2: Experimental conditions for emulsion AGET ATRP of BMA (Different Ligand System).

\begin{tabular}{|c|c|c|c|c|c|c|c|c|}
\hline Experiment & $\begin{array}{c}\text { BMA I } \\
(\mathrm{g})\end{array}$ & $\begin{array}{c}\mathrm{CuBr}_{2} \\
(\mathrm{~g})\end{array}$ & $\begin{array}{l}\text { Ligand } \\
\text { (g) }\end{array}$ & $\mathrm{H}_{2} \mathrm{O}(\mathrm{g})$ & $\begin{array}{c}\text { Brij } 98 \\
(\mathrm{~g})\end{array}$ & $\begin{array}{c}\text { EBIB } \\
(\mathrm{g})\end{array}$ & $\mathrm{AA}(\mathrm{g})$ & $\begin{array}{l}\text { BMA } \\
\text { II (g) }\end{array}$ \\
\hline PMDETA & 25 & 0.0819 & 0.1315 & 350 & 15.1623 & 0.55 & 0.05 & 50 \\
\hline HMTA & 25 & 0.0819 & 0.1068 & 350 & 15.1623 & 0.55 & 0.05 & 50 \\
\hline dNbpy & 25 & 0.0819 & 0.3111 & 350 & 15.1623 & 0.55 & 0.05 & 50 \\
\hline
\end{tabular}

The ligand PMDETA is a hydrophilic ligand that has been successfully used in bulk ATRP polymerization method. However, the rate of polymerization in aqueous media was so slow that almost no polybutyl-methacrylate was obtained. It is presumed this lack of reaction was due to the possibility of PMDETA diffused to the aqueous phase and was not able to bind coherently to the copper metal, which would initiate monomer polymerization. Hence, it failed to maintain the copper complex inside the polymerizing particles. Another possibility was that it did not provide sufficient active catalyst radicals in order to promote the polymerization of the butyl methacrylate under these conditions (Peng et al., 2003). Hence, this result points out ATRP polymerization of BMA in aqueous dispersed media cannot be similar to ATRP polymerization in bulk.

Since the polymerization did not occur with hydrophilic ligand, experimental trials using a hydrophobic ligand called 1,1,4,7,10,10-hexamethyldithylen-tetramine (HMTA) was attempted. Unfortunately, HMTA ligand caused to an uncontrolled polymerization. This is evident from the 
aggregation/coagulation experienced in the system (as shown in Figure 5.2) after $1 \mathrm{~h}$ of polymerization. The coagulation was due to poor stabilization of large polymer particles by the surfactant and the control of polymerization was lost.
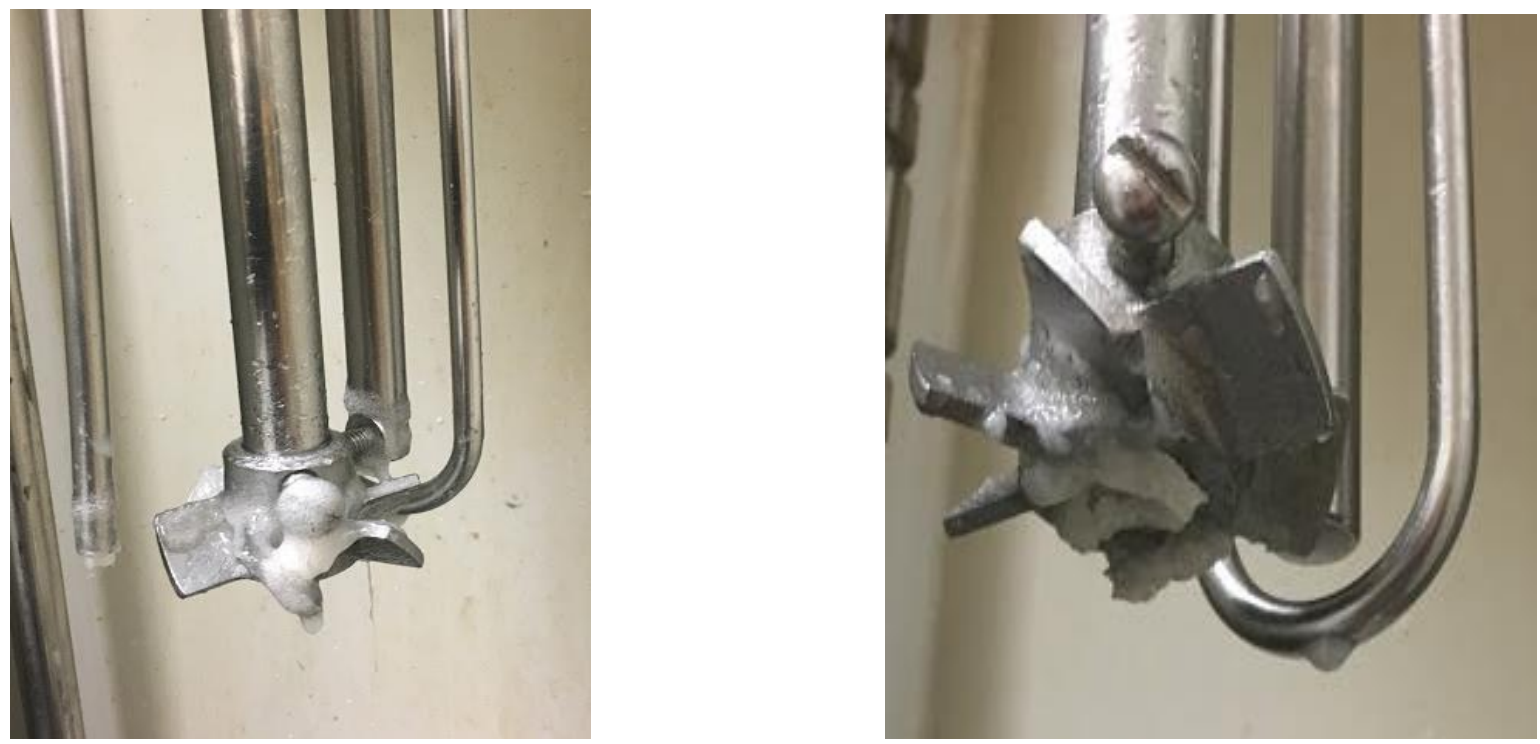

Figure 5.2: Aggregation/ Coagulation in AGET ATRP Emulsion System with HMTA as Ligand.

The third ligand dNbpy was used. dNbpy is a very hydrophobic ligand and has the characteristics to solubilize a large amount of copper complex inside the organic particles. It prevents the catalyst from diffusing out of the growing polymer particles (Peng and Cheng, 2005). Control of polymerization can be evaluated in terms of very low PDI (Mw/Mn) and a relatively low number average molecular weight. In this preliminary test, polymer samples obtained with dNbpy have narrow PDI (1.21) and low number average molecular weight (13879 g/mol). For this reaction condition at $50^{\circ} \mathrm{C}$, a low monomer conversion of $46.2 \%$ was obtained. Figure 5.3 showed the unimodal GPC traces for the polymerization. 


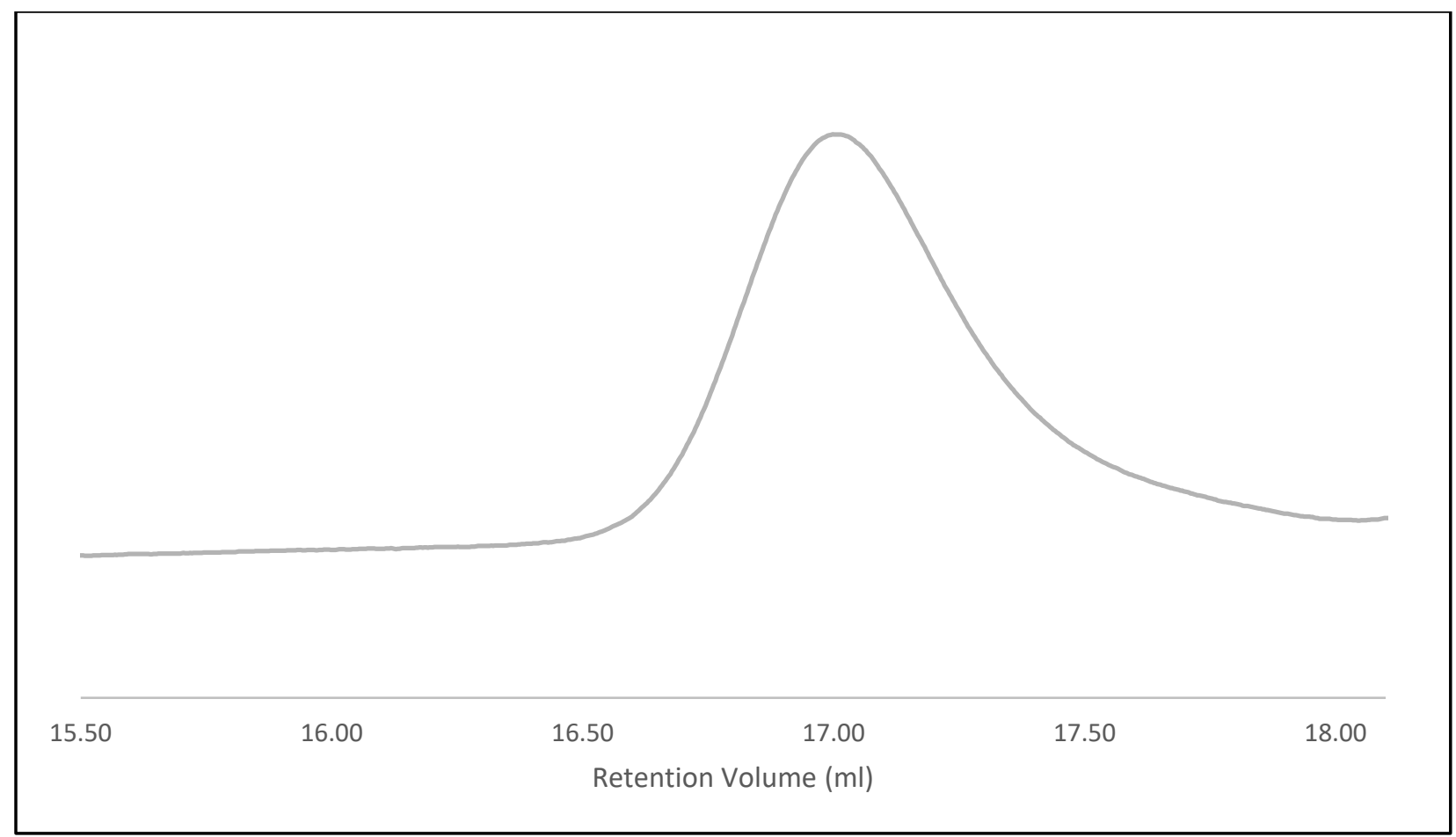

Figure 5.3: GPC traces for two-step emulsion polymerization with dNbpy ligand.

The ligand investigation conducted above confirms that not every ligand that is effective in bulk or solution ATRP can be successful in polymerize BMA in emulsion systems. In addition, the results obtained with dNbpy are reasonably well and can be used as a ground to conduct further experimental tests of AGET ATRP polymerization of BMA in emulsion reaction under various experimental conditions. The next section of this thesis is to address and establish an experimental plan of AGET ATRP in emulsion medium.

\subsubsection{Establishment of two-step polymerization method}

There are no guidelines to choose the experimental recipes and therefore, the next experiments were randomly done to determine the right proportion of reactants and reasonable reaction conditions using dNbpy as ligand. In this preliminary investigation, five experimental tests were performed under the conditions shown in Table 5.3. 
Table 5.3: Experimental condition for two-step emulsion AGET ATRP of BMA.

\begin{tabular}{|c|c|c|c|c|c|c|c|c|c|}
\hline Exp & $\begin{array}{c}\text { BMA } \\
(\mathrm{g})\end{array}$ & $\begin{array}{c}\text { EBiB } \\
(\mathrm{g})\end{array}$ & $\begin{array}{c}\text { Brij 98 } \\
(\mathrm{g})\end{array}$ & $\begin{array}{c}\text { dNbpy } \\
(\mathrm{g})\end{array}$ & $\begin{array}{c}\text { Ascorbic } \\
\text { Acid }(\mathrm{g})\end{array}$ & $\begin{array}{c}\text { CuBr } \\
(\mathrm{II})(\mathrm{g})\end{array}$ & $\begin{array}{c}\mathrm{H}_{2} \mathrm{O} \\
(\mathrm{g})\end{array}$ & Temp & $\begin{array}{c}\text { Time } \\
(\mathrm{min})\end{array}$ \\
\hline E1 & 79.5 & 0.5545 & 15.1623 & 0.3129 & 0.0542 & 0.0919 & 350 & 50 & 300 \\
\hline E2 & 79.5 & 0.5420 & 15.2687 & 0.3208 & 0.0563 & 0.0936 & 350 & 50 & 300 \\
\hline E3 & 67.1 & 0.5450 & 11.6189 & 0.3345 & 0.0546 & 0.0910 & 350 & 50 & 180 \\
\hline E4 & 53.4 & 0.5521 & 11.8910 & 0.3492 & 0.0532 & 0.0903 & 350 & 50 & 180 \\
\hline E5 & 53.4 & 0.2889 & 10.9120 & 0.1578 & 0.0562 & 0.0429 & 350 & 70 & 180 \\
\hline
\end{tabular}

For all five runs, polymerization was carried out under 20-psig pressure of nitrogen gas, stirring rate was 250rpm, and BMA II ( $2 / 3$ of the total BMA) was added after 12 min of initiation. Nitrogen purging was done during microemulsion for 3 min. The amount of AA was divided into two equal halves. The second half of AA (dissolve in $10 \mathrm{ml}$ of water) was added immediately after the addition of BMA II.

Table 5.4 of the experimental results, the data shows that the PDI obtained were fairly close in all experiments E1-E5. Hence, control of polymerization in terms of PDI (Mw/Mn) was realized. E2 was a replicate test of E1 to verify the repeatability of the experimental condition. The system was run at a low temperature of $50^{\circ} \mathrm{C}$ and high loading of reactants in attempt to result in more latex stability. Thus, aggregation/ coagulation was not experienced. However, monomer conversions in E1 $(46.2 \% / 5 \mathrm{~h})$ and E2 $(45.2 \% / 5 \mathrm{~h})$ are close to each other, but low. Experiment E3 and E4 were quite similar to E1 and E2, except that the polymerization period was shorter, and both the monomer and surfactant load were decreased. But, latex stability and monomer conversion for E3 (45.9\%/3h) and E4 (42.8\%/3h) were not quite satisfactory. To improve the reaction condition, experiment $\mathrm{E} 5$, with the same amount of BMA as $\mathrm{E} 4$ was run at high temperature of $70^{\circ} \mathrm{C}$ but halving each amount of the initiator (EBib), catalyst $\left(\mathrm{CuBr}_{2}\right)$ and ligand (dNbpy). No coagulation in E5 was observed despite a higher conversion of $61.2 \%$ was obtained. Figure 5.4 shows that the MWD of PBMA under conditions of E5 is quite broad even though the conversion is high. Consequently, obtaining a well-controlled polymerization with higher conversion in E5 will be used to investigate the different factors affecting the latex stability in two-step emulsion of AGET ATRP of BMA 
Table 5.4: Experimental results for two-step emulsion AGET ATRP of BMA.

\begin{tabular}{|c|c|c|c|c|}
\hline Exp & $t(\min )$ & Conv (\%) & $\mathrm{Mn}, \mathrm{GPC}(\mathrm{g} / \mathrm{mol})$ & PDI \\
\hline \multirow[t]{3}{*}{$\mathrm{E} 1$} & 60 & 17.3 & 4189 & 1.502 \\
\hline & 180 & 35.4 & 9890 & 1.321 \\
\hline & 300 & 46.2 & 13878 & 1.218 \\
\hline \multirow[t]{3}{*}{ E2 } & 60 & 19.2 & 5370 & 2.097 \\
\hline & 180 & 34.3 & 11042 & 1.894 \\
\hline & 300 & 45.2 & 14820 & 1.409 \\
\hline \multirow[t]{3}{*}{ E3 } & 60 & 22.7 & 19980 & 1.191 \\
\hline & 120 & 40.4 & 23789 & 1.263 \\
\hline & 180 & 45.9 & 24988 & 1.211 \\
\hline \multirow[t]{3}{*}{ E4 } & 60 & 25.3 & 10930 & 1.242 \\
\hline & 120 & 37.5 & 16789 & 1.285 \\
\hline & 180 & 42.8 & 21989 & 1.206 \\
\hline \multirow[t]{3}{*}{ E5 } & 60 & 30.5 & 35672 & 1.308 \\
\hline & 120 & 56.3 & 41276 & 1.229 \\
\hline & 180 & 61.2 & 45607 & 1.285 \\
\hline
\end{tabular}

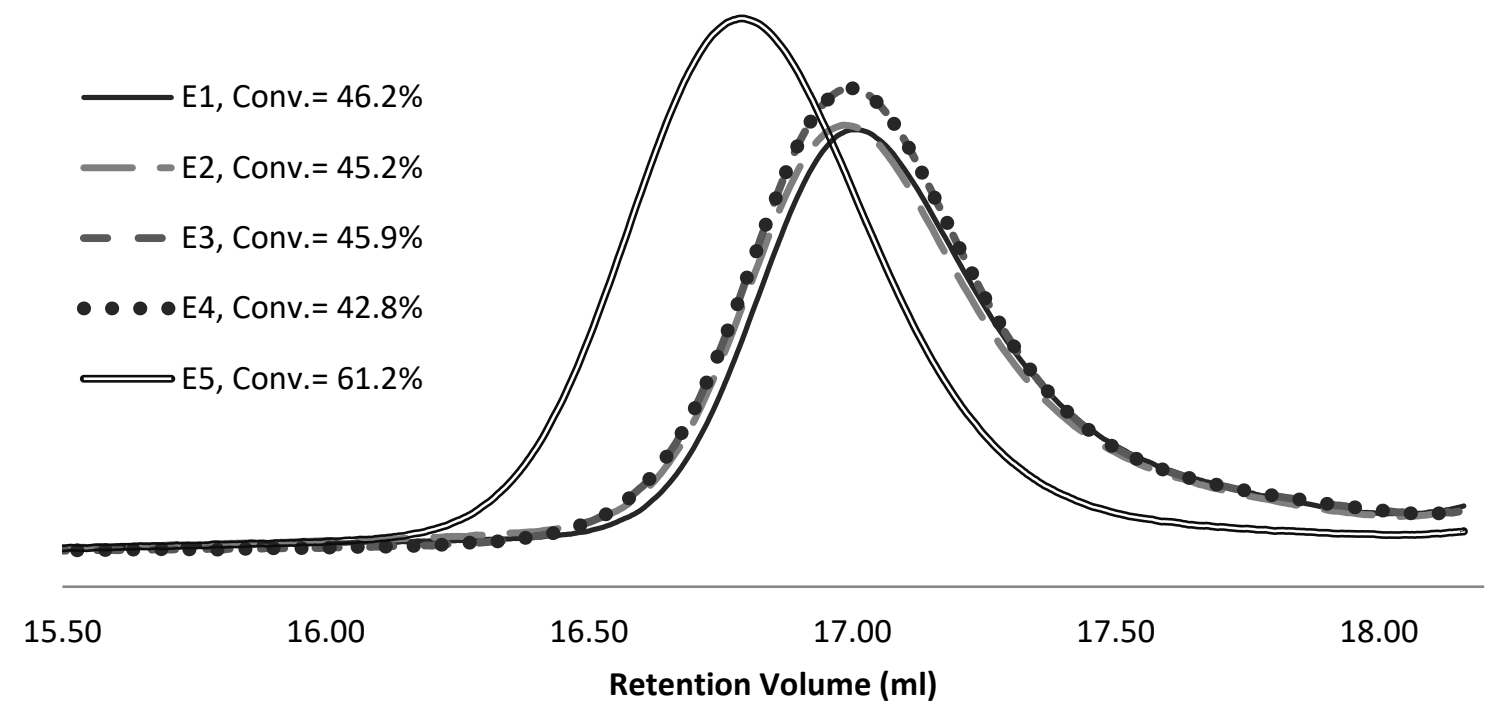

Figure 5.4: GPC traces for two-step emulsion polymerization preliminary investigation. 


\subsubsection{Conclusion}

AGET ATRP has been successfully applied using a two-step procedure emulsion polymerization, in which microemulsion was formed with presence of air. When a hydrophilic ligand PMDETA was used, the polymerization was too slow, and no polymerization was observed after $5 \mathrm{~h}$. However, polymerization with the hydrophobic HMTA ligand was not successful. When a hydrophobic ligand dNbpy was used to polymerize BMA; PBMA polymer was successfully synthesized with an average molecular weight of $13879 \mathrm{~g} / \mathrm{mol}$ and a PDI of 1.3 . 


\section{CHAPTER 6}

\section{INVESTIGATION OF AGET ATRP OF BMA IN EMULSION POLYMERIZATION}

Considering the very limited information available on the experimental recipient of AGET ATRP

of BMA in emulsion systems, several experiments were carried out to determine reasonable initial molar concentration of the reactants for the reaction to proceed. It was demonstrated that successful polymerization of BMA using AGET ATRP can be conducted in aqueous dispersed media specifically through two-step polymerization procedure. Due to the heterogeneous nature of the emulsion system, the kinetics of the exchange between the active and dormant species (a key factor in obtaining a controlled living polymerization) becomes more complicated than in the organic homogeneous system. In order to maintain the "living" character of the polymerization in a stable dispersed system, several factors need to be considered. In this section below, each key variable of the system has been examined to investigate its effect on butyl methacrylate polymerization. The key variables considered are the polymerization temperature, ligand, surfactant and reducing agent.

\subsection{Temperature}

Thermal energy required to start and maintain the reaction plays an important role in rate of polymerization. In general, a high temperature leads to a high radical concentration and disfavours the organic partitioning of the deactivators. As a result, excessive polymer termination can occur leading to a gradual buildup of deactivator and consequently, it may slow down the polymerization rate. As shown in Table 6.1, three experiment trials were done for temperatures of $50^{\circ} \mathrm{C}, 60^{\circ} \mathrm{C}$ and $70^{\circ} \mathrm{C}$. For all runs, the polymerization was carried out under 20 -psig pressure of nitrogen gas, the stirring rate was kept constant at 250rpm, and the second amount of BMA was added after 12min of reaction start-up. Nitrogen purging was done only for the microemulsion period for about $5 \mathrm{~min}$. The amount of ascorbic acid (AA) was equally halved. The second half of AA was dissolved in 10 $\mathrm{ml}$ of distilled water and then was added immediately after the addition of BMA. 
Table 6.1: AGET ATRP emulsion polymerization using two-step technique for different temperatures.

\begin{tabular}{|c|r|r|r|r|r|r|r|r|r|}
\hline Exp\# & $\begin{array}{c}\text { Temp } \\
{ }^{\circ} \mathbf{C}\end{array}$ & $\begin{array}{c}\text { BMA } \\
\mathrm{I}(\mathrm{g})\end{array}$ & $\begin{array}{c}\mathrm{CuBr}_{2} \\
(\mathrm{~g})\end{array}$ & $\begin{array}{c}\text { dNbpy } \\
(\mathrm{g})\end{array}$ & $\begin{array}{c}\mathrm{H}_{2} \mathrm{O} \\
(\mathrm{g})\end{array}$ & $\begin{array}{c}\text { Brij98 } \\
(\mathrm{g})\end{array}$ & $\begin{array}{c}\text { EBiB } \\
(\mathrm{g})\end{array}$ & $\begin{array}{c}\text { AA } \\
(\mathrm{g})\end{array}$ & $\begin{array}{c}\text { BMA } \\
2(\mathrm{~g})\end{array}$ \\
\hline $\mathrm{R} 1$ & $\mathbf{5 0}$ & 8.94 & 0.0418 & 0.1496 & 350 & 10.2300 & 0.3349 & 0.0506 & 44.5 \\
\hline $\mathrm{R} 2$ & $\mathbf{6 0}$ & 8.94 & 0.0425 & 0.1497 & 350 & 10.2469 & 0.3429 & 0.0507 & 44.5 \\
\hline $\mathrm{R} 3$ & $\mathbf{7 0}$ & 8.94 & 0.0430 & 0.1513 & 350 & 10.2353 & 0.3278 & 0.0523 & 44.5 \\
\hline
\end{tabular}

Table 6.2: Experimental results of PBMA by a Two-step emulsion AGET ATRP using different temperatures.

\begin{tabular}{ccccc}
\hline Exp & Time (min) & Conversion $(\%)$ & GPC, Mn $(\mathrm{g} / \mathrm{mol})$ & PDI \\
\hline R1 & 60 & 37.9 & 3625 & 1.307 \\
& 120 & 49.3 & 5567 & 1.252 \\
& 180 & 63.9 & 6537 & 1.338 \\
R2 & 60 & 45.6 & 3445 & 1.432 \\
& 120 & 60.9 & 4317 & 1.419 \\
R3 & 180 & 70.2 & 5681 & 1.224 \\
& 60 & 52.7 & 5762 & 1.549 \\
& 120 & 73.9 & 7965 & 1.304 \\
& 180 & 85.8 & 9185 & 1.265
\end{tabular}

Table 6.2 shows the gravimetry and GPC analysis. In all three experiments, the monomer conversion exceeded $60 \%$ during $3 \mathrm{~h}$ of reaction time. The conversion reached a high value of $85.8 \%$ at $70^{\circ} \mathrm{C}$, but the MWD was the narrowest at $60^{\circ} \mathrm{C}$ with value of 1.224 . The polymer samples have a Mn range between 5681 and $9185 \mathrm{~g} / \mathrm{mol}$ and a PDI ranging from 1.224 to 1.338 which corresponds to a well-controlled polymerization. The Plots in Figure 6.1 show that the GPC traces with a low-molecular weight tail at $50^{\circ} \mathrm{C}$ and $60^{\circ} \mathrm{C}$, but a unimodal MWD plot for experiment run 
R3. A possible reason for the MWD tailing is that large number of active chains were formed at the beginning of the polymerization leading to radical termination and thus the formation of lowmolecular-weight dead chains.

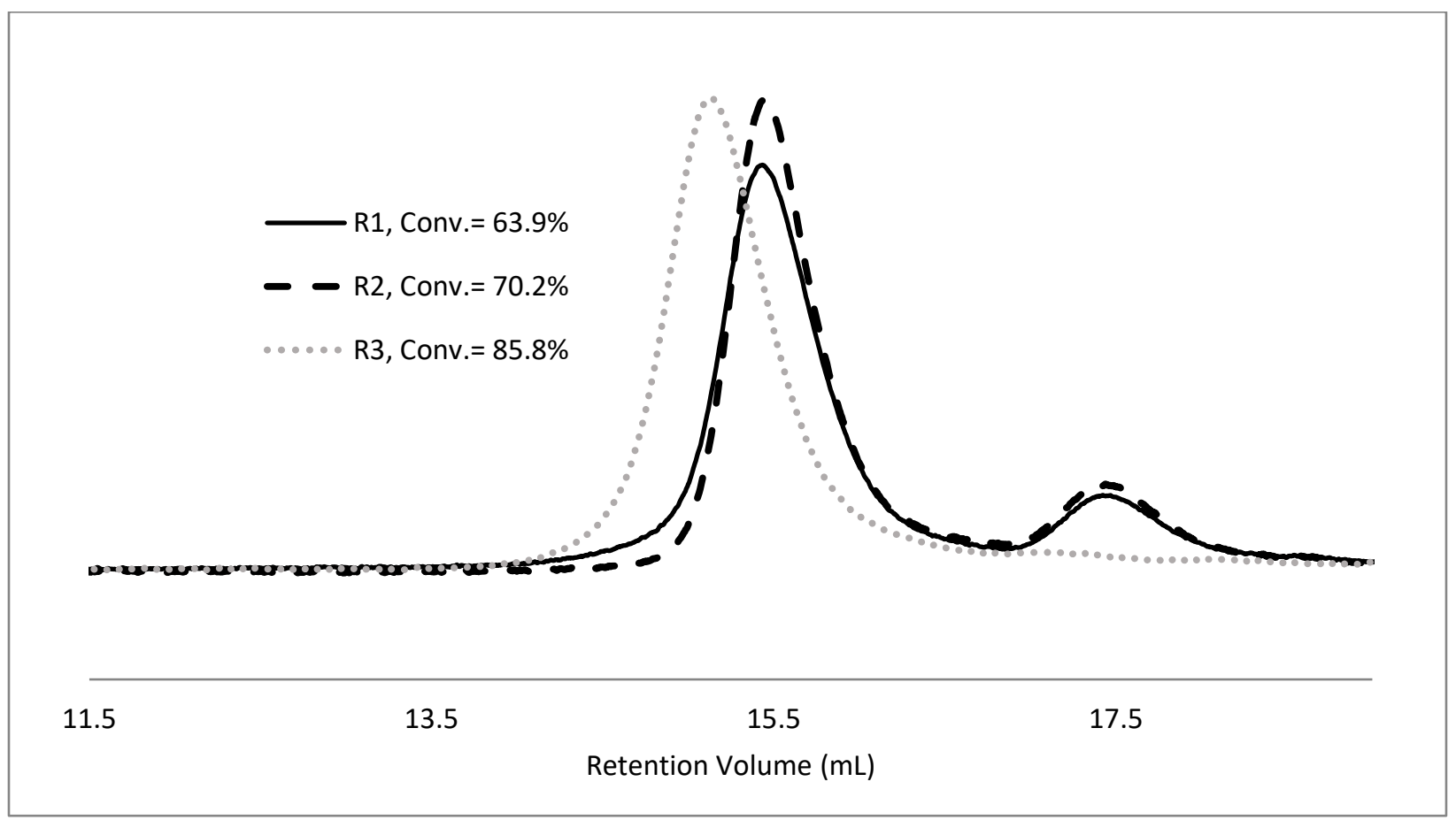

Figure 6.1: $\mathrm{GPC}$ traces of the effect of temperature at $50^{\circ} \mathrm{C}, 60^{\circ} \mathrm{C}$ and $70^{\circ} \mathrm{C}$ on BMA polymerization at 180min [BMA]:[EBiB]:[CuBr 2$]:[\mathrm{dNbpy}]:[\mathrm{AA}]:[\mathrm{Brij} 98]=219: 1: 0.11: 0.21: 0.17: 5.18$.

Figure 6.2 shows BMA conversion variations versus time for different reaction temperatures. Trends of monomer conversion profiles increase steadily, and they show no diffusion effect as it is usually observed in conventional free radical polymerization. In addition, the plot indicates a high initial conversion of BMA emulsion polymerization for all three temperatures. Relatively higher initial conversion of $52.7 \%$ was obtained at $70^{\circ} \mathrm{C}$. First-order kinetic plots in Figure 6.3 show a linear trend which demonstrates living/controlled features of the polymer. 


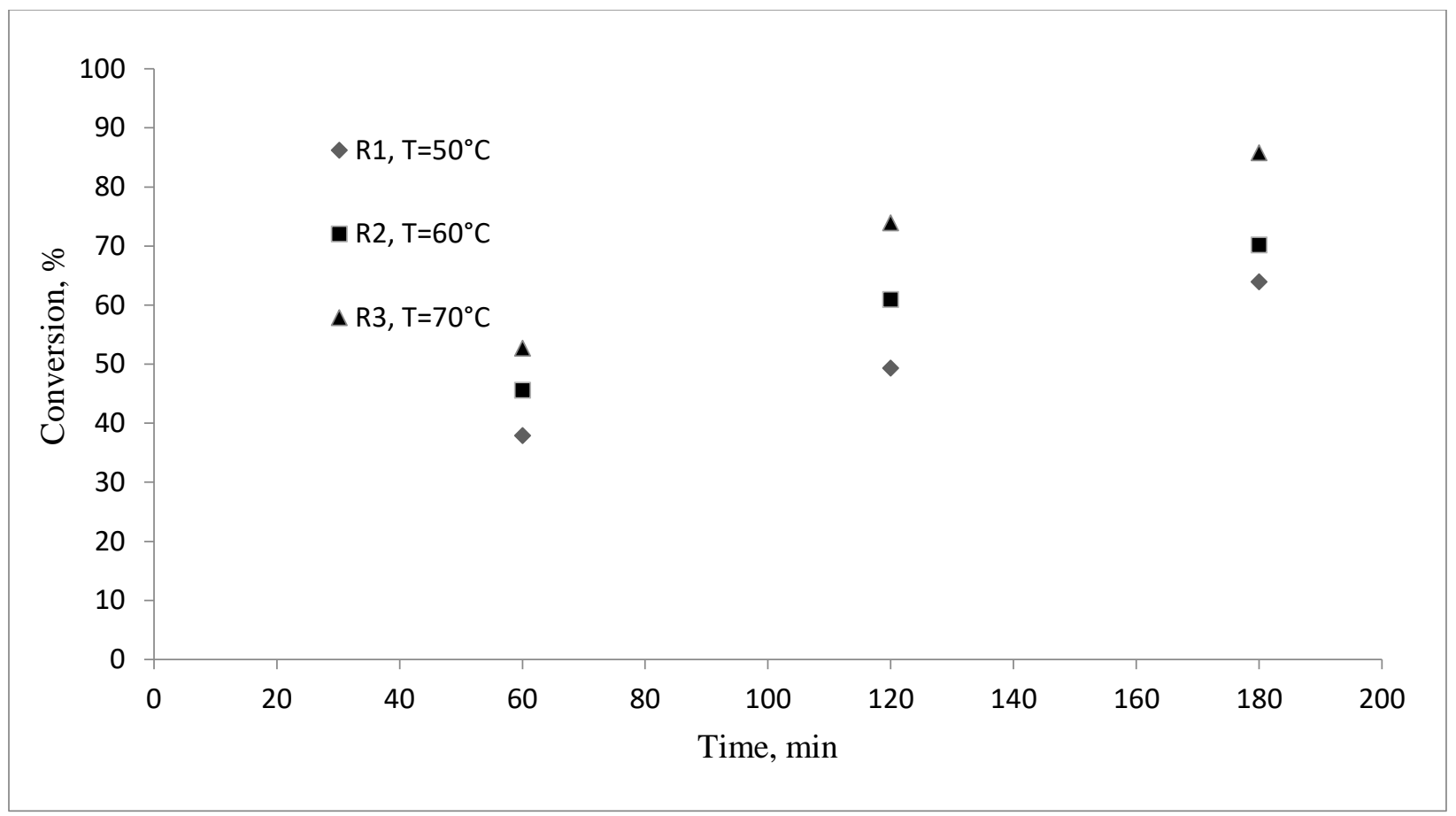

Figure 6.2: Conversion versus time for different reaction temperature $\left(50^{\circ} \mathrm{C}, 60^{\circ} \mathrm{C}\right.$ and $\left.70^{\circ} \mathrm{C}\right)$. [BMA]:[EBiB]:[CuBr 2$]:[\mathrm{dNbpy}]:[\mathrm{AA}]:[\mathrm{Brij} 98]=219: 1: 0.11: 0.21: 0.17: 5.18$.

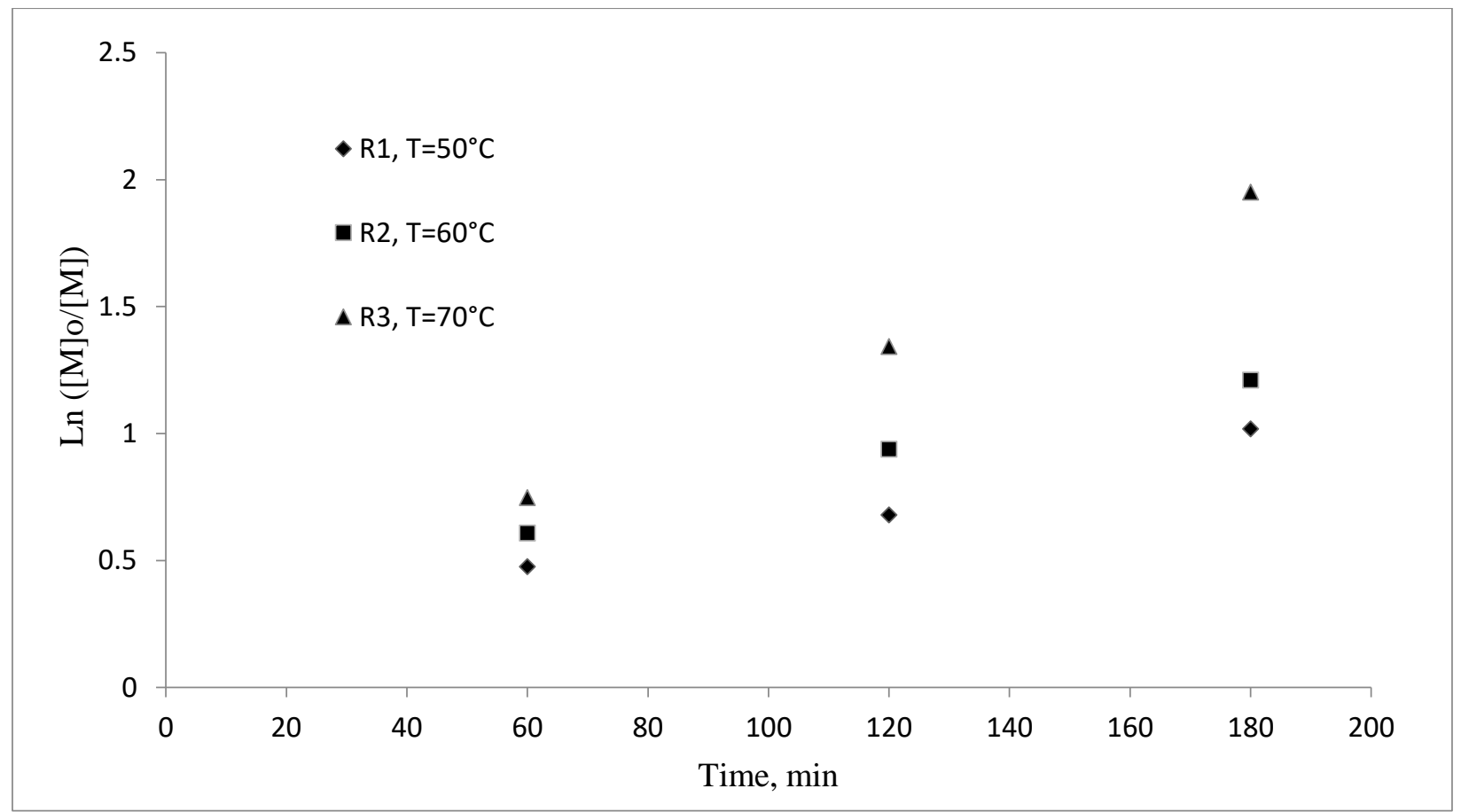

Figure 6.3: First order kinetics plot of BMA versus time for different reaction temperature $\left(50^{\circ} \mathrm{C}, 60^{\circ} \mathrm{C}\right.$ and $\left.70^{\circ} \mathrm{C}\right)$. [BMA]:[EBiB]:[CuBr 2$]:[\mathrm{dNbpy}]:[\mathrm{AA}]:[$ Brij 98]= 219:1:0.11:0.21:0.17:5.18. 


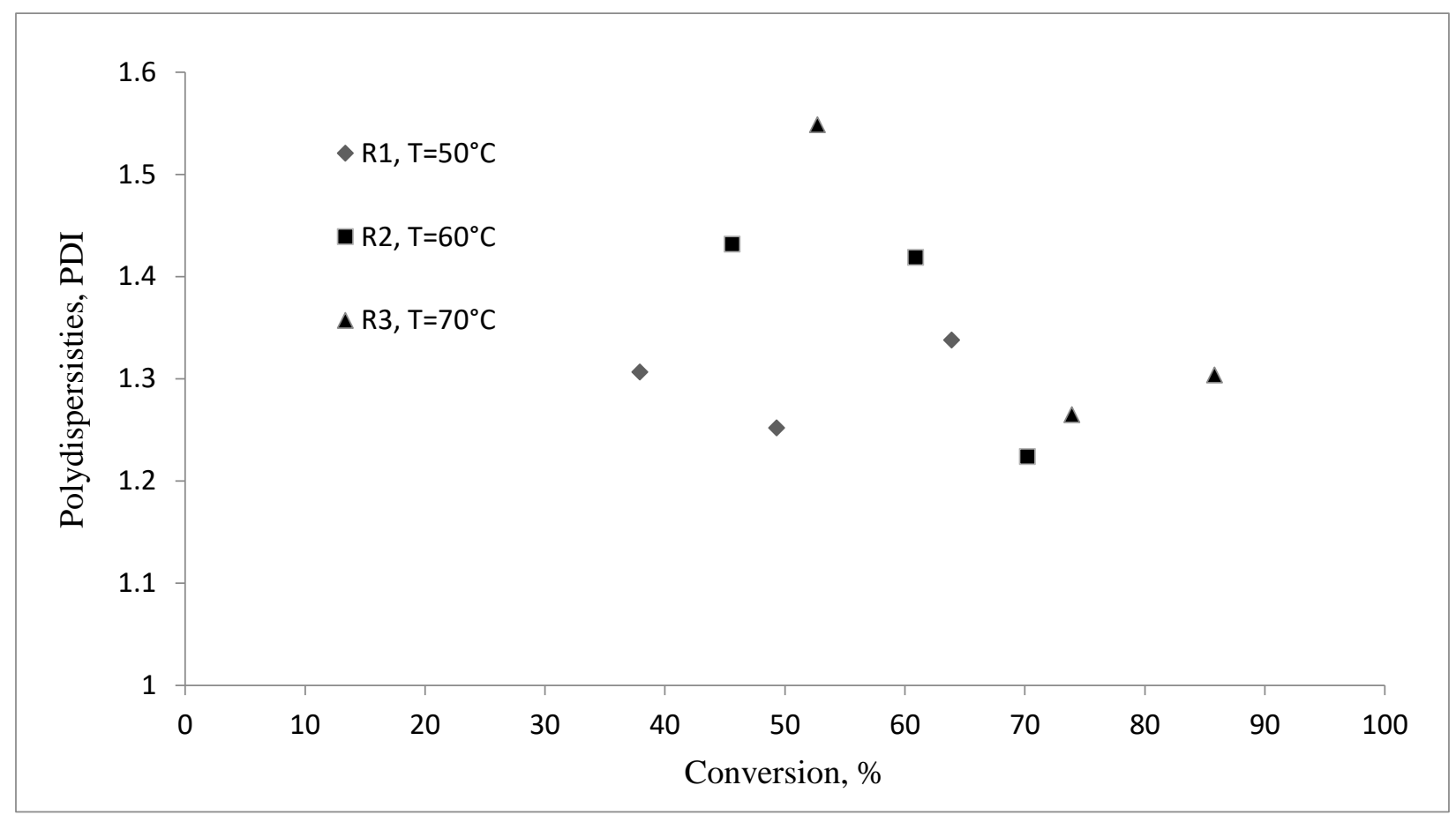

Figure 6.4: PDI versus conversion plot for different reaction temperature $\left(50^{\circ} \mathrm{C}, 60^{\circ} \mathrm{C}\right.$ and $\left.70^{\circ} \mathrm{C}\right)$. [BMA]:[EBiB]:[CuBr 2$]:[\mathrm{dNbpy}]:[\mathrm{AA}]:[\mathrm{Brij} 98]=219: 1: 0.11: 0.21: 0.17: 5.18$.

PDI data of PBMA samples are plotted in Figure 6.4, which shows that the MWD narrows as the reaction progresses. The PDI decreasing plots confirms that the uniformity of chain length at higher conversion. Figure 6.5 presents number average molecular weights $(\mathrm{Mn})$ versus BMA conversion for the three experimental trials. High $\mathrm{Mn}$ of $9185 \mathrm{~g} / \mathrm{mol}$ was obtained for R3, while the Mn of polymer samples collected in R1 and R2 were 6537 and 5681g/mol respectively. 


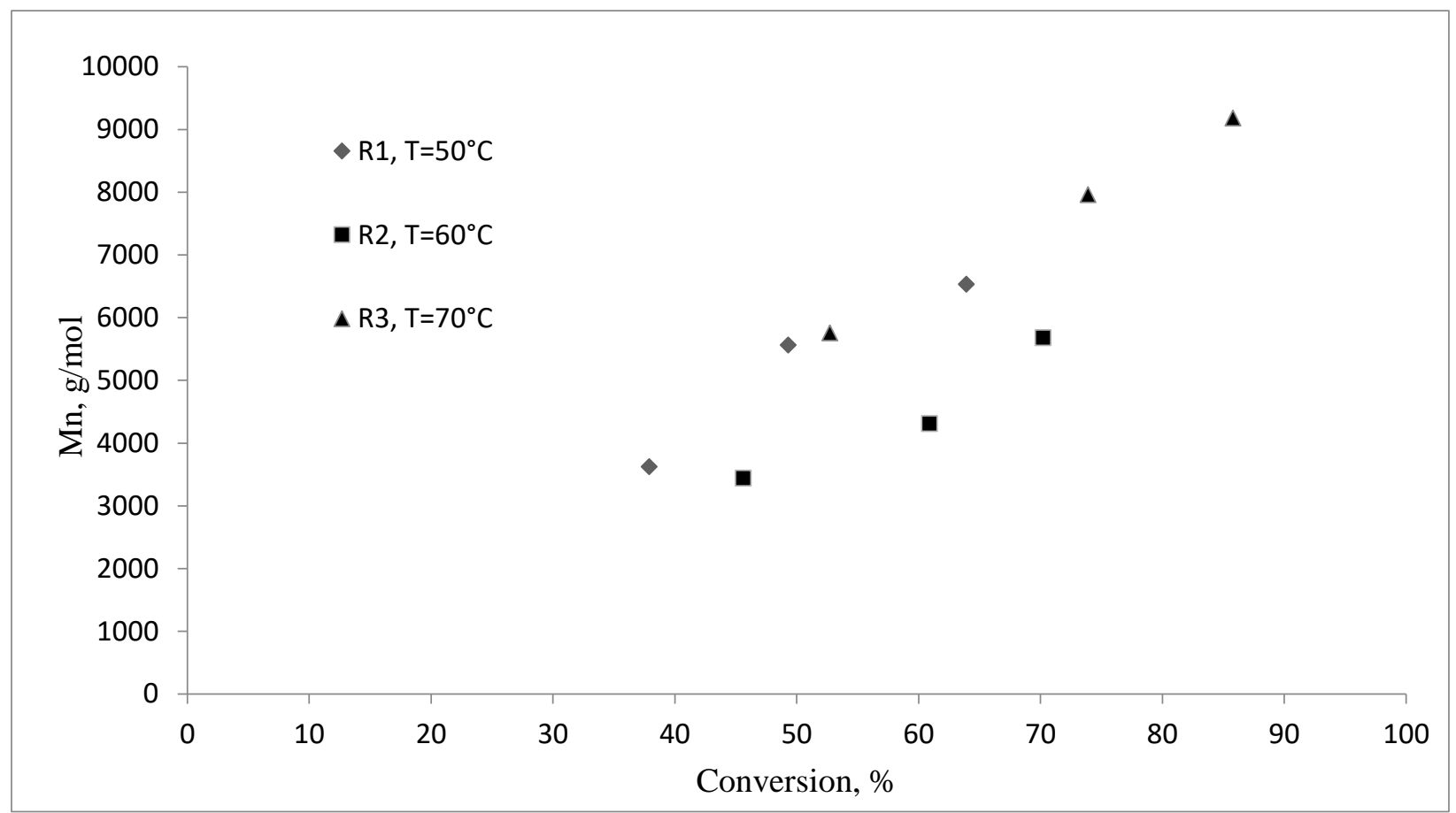

Figure 6.5: Number average molecular weight vs conversion for different reaction temperature $\left(50^{\circ} \mathrm{C}, 60^{\circ} \mathrm{C}\right.$ and $\left.70^{\circ} \mathrm{C}\right)$. [BMA]:[EBiB]:[CuBr 2$]:[\mathrm{dNbpy}]:[\mathrm{AA}]:[\mathrm{Brij} 98]=219: 1: 0.11: 0.21: 0.17: 5.18$.

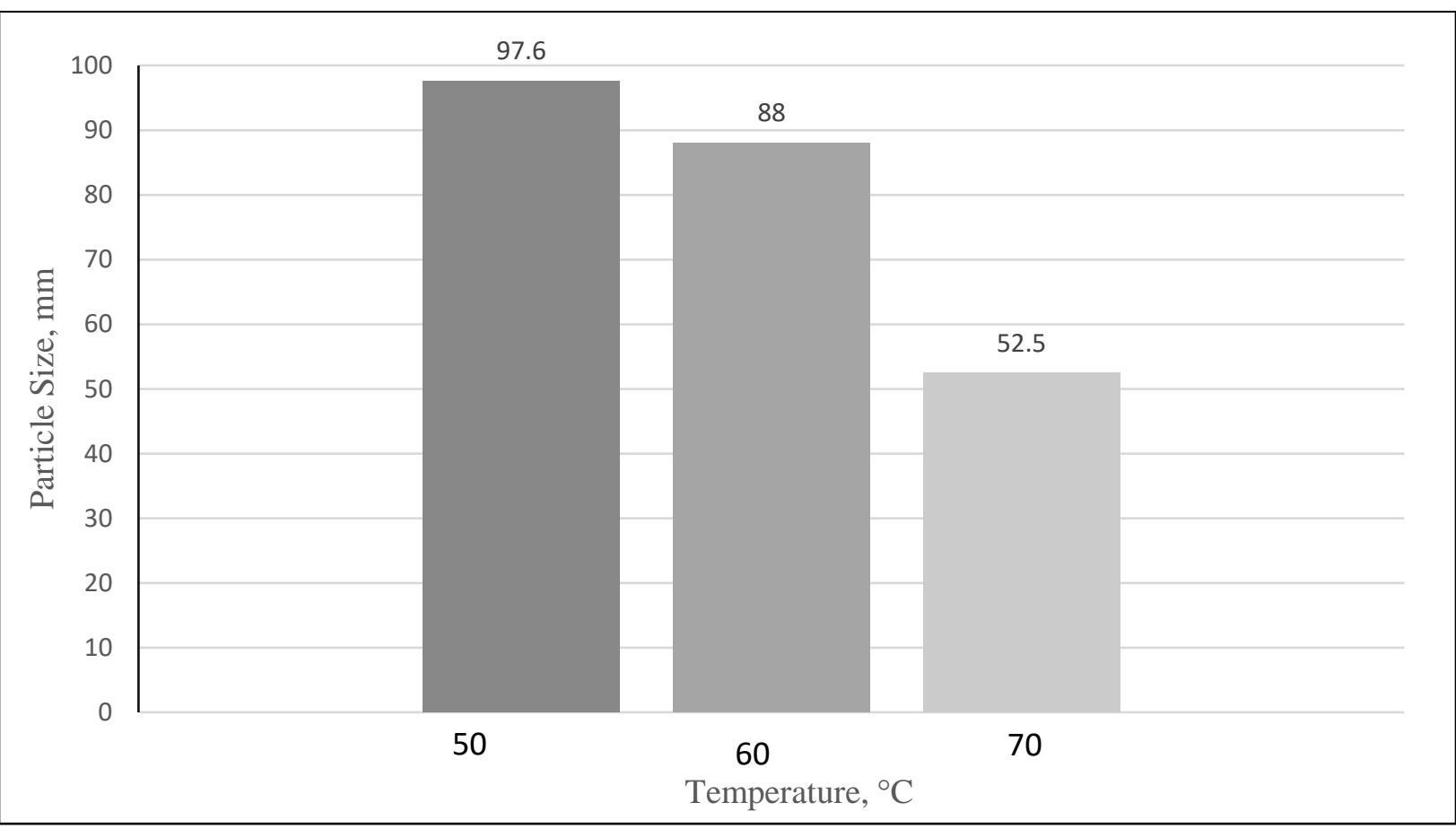

Figure 6.6: Particle size at $180 \mathrm{~min}$ at different temperature: $50^{\circ} \mathrm{C}, 60^{\circ} \mathrm{C}$ and $70^{\circ} \mathrm{C}$. [BMA]:[EBiB]:[CuBr 2$]:[\mathrm{dNbpy}]:[\mathrm{AA}]:[$ Brij 98]= 219:1:0.11:0.21:0.17:5.18. 
Reaction temperature affects the particle size of PBMA polymers as shown in Figure 6.6. The size of the latex particle decreased from $97.6 \mathrm{~nm}$ to $52.5 \mathrm{~nm}$, as the temperature varied from $50^{\circ} \mathrm{C}$ to $70^{\circ} \mathrm{C}$. The rate of propagating radical generation increased with temperature and resulted in significant augmentation of radical concentration. The polymerization rate increased the growing polymer radical to the emulsion particle from the aqueous phase which induced the enhanced nucleation rate. As a result, number of the particles increased, and the particle size decreased.

\subsection{Ligand/ Catalyst Complex}

The selection of a suitable catalyst in ATRP is important to maintain a dynamic equilibrium between the growing polymer radicals and dormant species. The catalyst ensures fast initiation which reduces the chance of radical termination and contributes to controlled/living characteristics of the polymerization. Also, the ligand in the metal catalyst complex plays another crucial role as it enhances the solubility of the catalyst complex and consequently the concentration of the activator and deactivator in the system (Qiu et al., 1999; Li and Matyjaszewski, 2003). In addition, it may shift the reaction equilibrium through steric effects. Both the ligand and catalyst impact considerably on the polymerization rate kinetics and the polymer chain length control. In copper mediated ATRP, a variety of ligands have been successfully employed for producing polymers with desired molecular weights, narrow molecular weight distributions, easily tailored compositions, and architectures (Peng et al., 2003; Min et al., 2006).

In an aqueous dispersed system, additional physio-chemical features of the polymerization need to be addressed to determine an appropriate catalytic system: (i) the ligand should have sufficient binding affinity towards the metal in order to compete with the water molecule as a potential ligand and (ii) should be able to solubilize enough metal complex (both the $\mathrm{Cu}^{\mathrm{I}}$ and $\mathrm{Cu}^{\mathrm{II}}$ in the organic phase wherein the polymerization take place) so that essential equilibrium for ATRP can be established (Shipp et al., 1998; Peng and Cheng, 2005; Min et al., 2006). As foresaid, not every ligand that was effectively used in bulk or solution ATRP continues to be successful in emulsion system. In the set of experimental tests (Table 6.3), the initial amounts of all reactants were kept invariant, except the initial amount of the ligand which was varied to demonstrate the effect of dNbpy ligand concentration on the AGET ATRP of BMA in aqueous medium. Three experimental runs R4 to R6 of BMA polymerization were carried out under 20-psig pressure of nitrogen gas. 
The medium was stirred at $250 \mathrm{rpm}$ and the second amount of BMA was added after the first 12 min of reaction. Similar to previous trials, nitrogen purging was done only during the microemulsion polymerization for only $5 \mathrm{~min}$. The initial amount of AA was split into two equal halves. The second half of AA was dissolved in $10 \mathrm{ml}$ of distilled water and then was added immediately after the addition of BMA.

Table 6.3: AGET ATRP emulsion polymerization using two-step technique for different ligand/catalyst ratios.

\begin{tabular}{|c|r|c|c|c|c|c|c|r|r|}
\hline Exp\# & $\begin{array}{c}\text { BMA } \\
\mathrm{I}(\mathrm{g})\end{array}$ & $\begin{array}{c}\mathrm{CuBr}_{2} \\
(\mathrm{~g})\end{array}$ & $\begin{array}{c}\text { dNbpy } \\
(\mathbf{g})\end{array}$ & $\begin{array}{c}\mathrm{H}_{2} \mathrm{O} \\
(\mathrm{g})\end{array}$ & $\begin{array}{c}\text { Brij 98 } \\
(\mathrm{g})\end{array}$ & $\begin{array}{c}\text { EBiB } \\
(\mathrm{g})\end{array}$ & AA (g) & $\begin{array}{c}\text { BMA 2 } \\
(\mathrm{g})\end{array}$ & $\begin{array}{c}\text { Ligand/Catalyst } \\
\text { (mol ratio) }\end{array}$ \\
\hline R4 & 8.94 & 0.0427 & $\mathbf{0 . 0 7 7 9}$ & 350 & 10.4380 & 0.3422 & 0.0517 & 44.5 & $1: 1$ \\
\hline R5 & 8.94 & 0.0461 & $\mathbf{0 . 1 5 0 1}$ & 350 & 10.4180 & 0.3526 & 0.0518 & 44.5 & $2: 1$ \\
\hline R6 & 8.94 & 0.0439 & $\mathbf{0 . 2 3 7 8}$ & 350 & 10.4195 & 0.3324 & 0.0516 & 44.5 & $3: 1$ \\
\hline
\end{tabular}

Table 6.4: Experimental result of PBMA by a Two-step emulsion AGET ATRP of BMA using different ligand/catalyst ratios.

\begin{tabular}{ccccc}
\hline Exp & Time $(\mathrm{min})$ & Conversion $(\%)$ & $\begin{array}{c}\text { GPC }, \mathrm{Mn} \\
(\mathrm{g} / \mathrm{mol})\end{array}$ & PDI \\
\hline R4 & 60 & 56.6 & 5865 & 1.649 \\
& 120 & 71.2 & 8095 & 1.452 \\
& 180 & 83.9 & 10450 & 1.368 \\
$\mathrm{R} 5$ & 60 & 45.3 & 3305 & 1.217 \\
& 120 & 72.1 & 7735 & 1.259 \\
& 180 & 79.6 & 9097 & 1.248 \\
& 60 & 52.7 & 3669 & 1.247 \\
& 120 & 65.9 & 6876 & 1.255 \\
& 180 & 75.7 & 8472 & 1.119
\end{tabular}




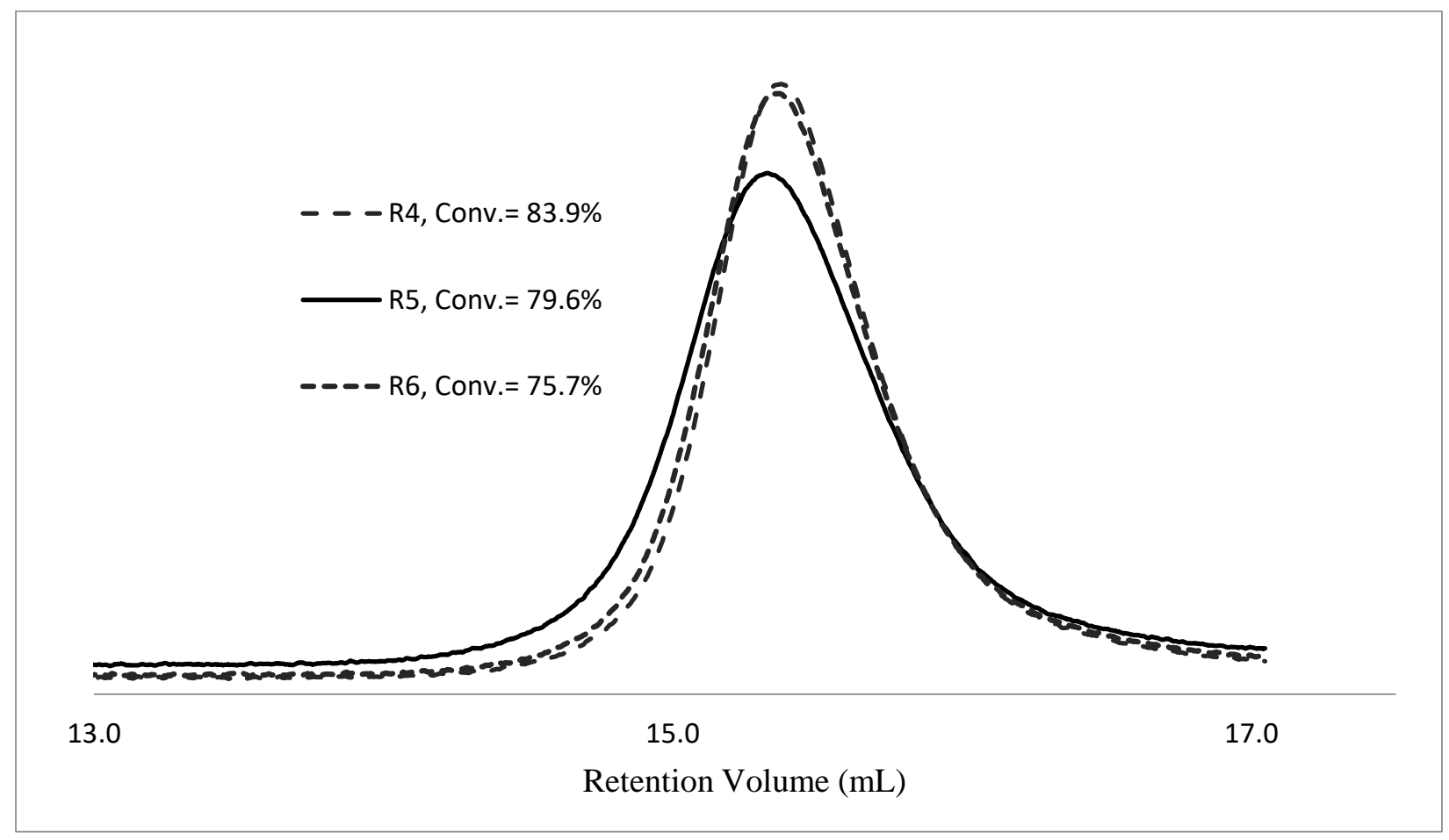

Figure 6.7: GPC traces of the effect of ligand concentration on BMA polymerization at $180 \mathrm{~min}$. $[\mathrm{dNbpy}] /\left[\mathrm{CuBr}_{2}\right]=$ 1, 1.5, and 3 respectively. [BMA]:[EBiB]:[CuBr 2$]:[\mathrm{AA}]:\left[\right.$ Brij98] $=213: 1: 0.12: 0.17: 5.15$, Reaction temp $=70^{\circ} \mathrm{C}$.

According to the ATRP mechanism (Shipp et al., 1998; Teodorescu and Matyjaszewski, 1999; Inoue and Matyjaszewski, 2004), the $\mathrm{CuBr}_{2}$ concentration affects considerably the production of polymer live radicals, polymerization rate is accelerated, and monomer conversion also increases. However, excess propagating radicals cause faster termination and broader MWD is demonstrated. Table 6.4 shows the gravimetry and GPC analysis. Experiments showed that monomer conversions reached approximately $80 \%$ in 3 h producing narrow MWD polymer samples with PDI of 1.368, 1.248 and 1.119 (Figure 6.7).

Figure 6.8 shows BMA conversion versus time for different ligand/catalyst ratio. The trends of monomer conversion profiles increase steadily. In addition, first order kinetic plots $\left(\mathrm{Ln}\left([\mathrm{M}]_{\mathrm{o}} /[\mathrm{M}]\right)\right.$ versus Time) illustrated in Figure 6.9 showed a linear plot indicating a controlled feature of the BMA polymerization in emulsion system. Figure 6.10 showed that as the $\mathrm{CuBr}_{2}$ concentration increases in R4, the monomer conversion increases and MWD becomes broader in comparison to R5 and R6. 


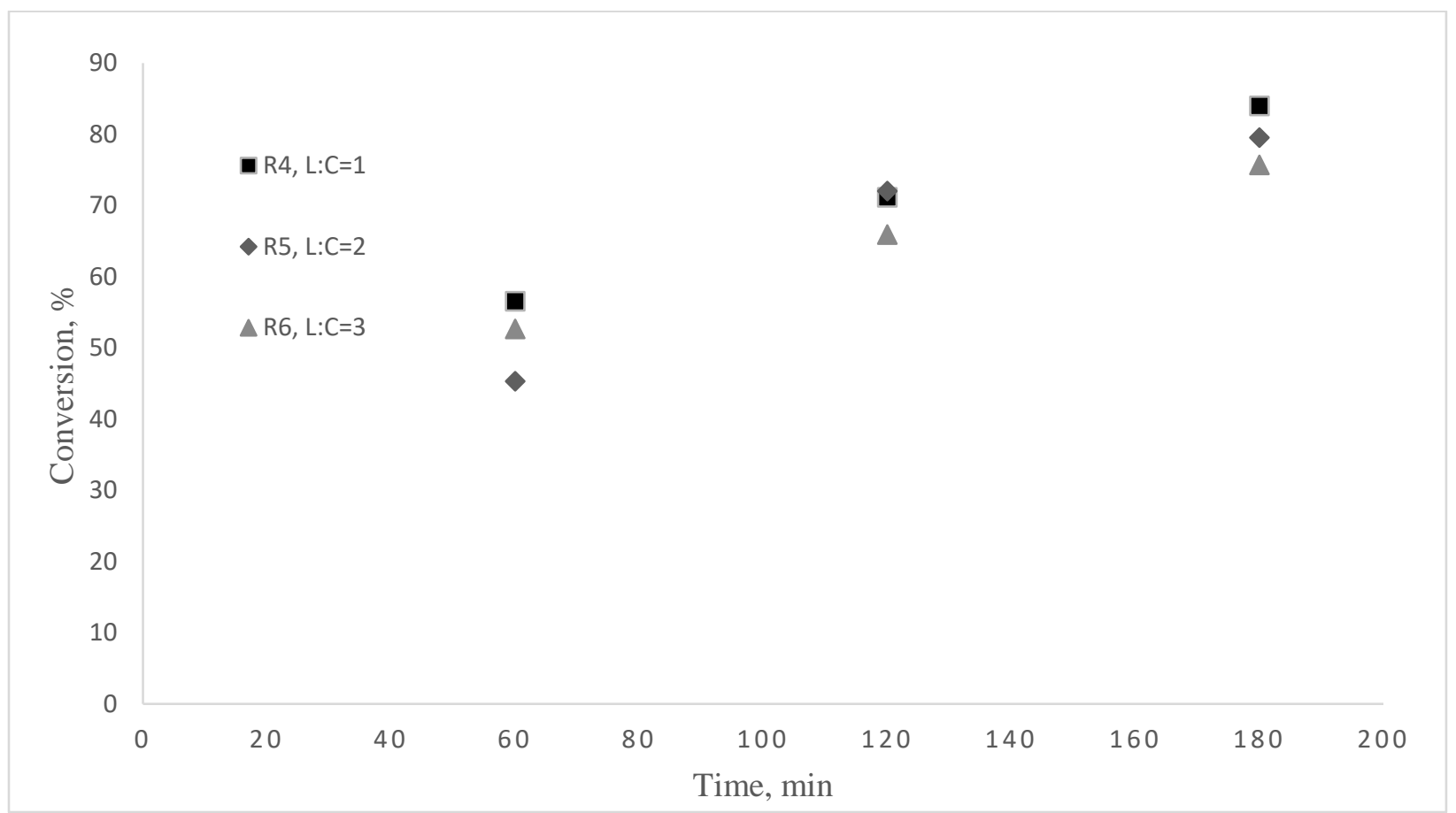

Figure 6.8: Conversion versus time for different catalyst/ligand concentration. [dNbpy] $/\left[\mathrm{CuBr}_{2}\right]=1,1.5$ and 3 respectively. [BMA]:[EBiB]:[ $\left.\mathrm{CuBr}_{2}\right]:[\mathrm{AA}]:[\mathrm{Brij} 98]=213: 1: 0.12: 0.17: 5.15$, Reaction temp $=70^{\circ} \mathrm{C}$.

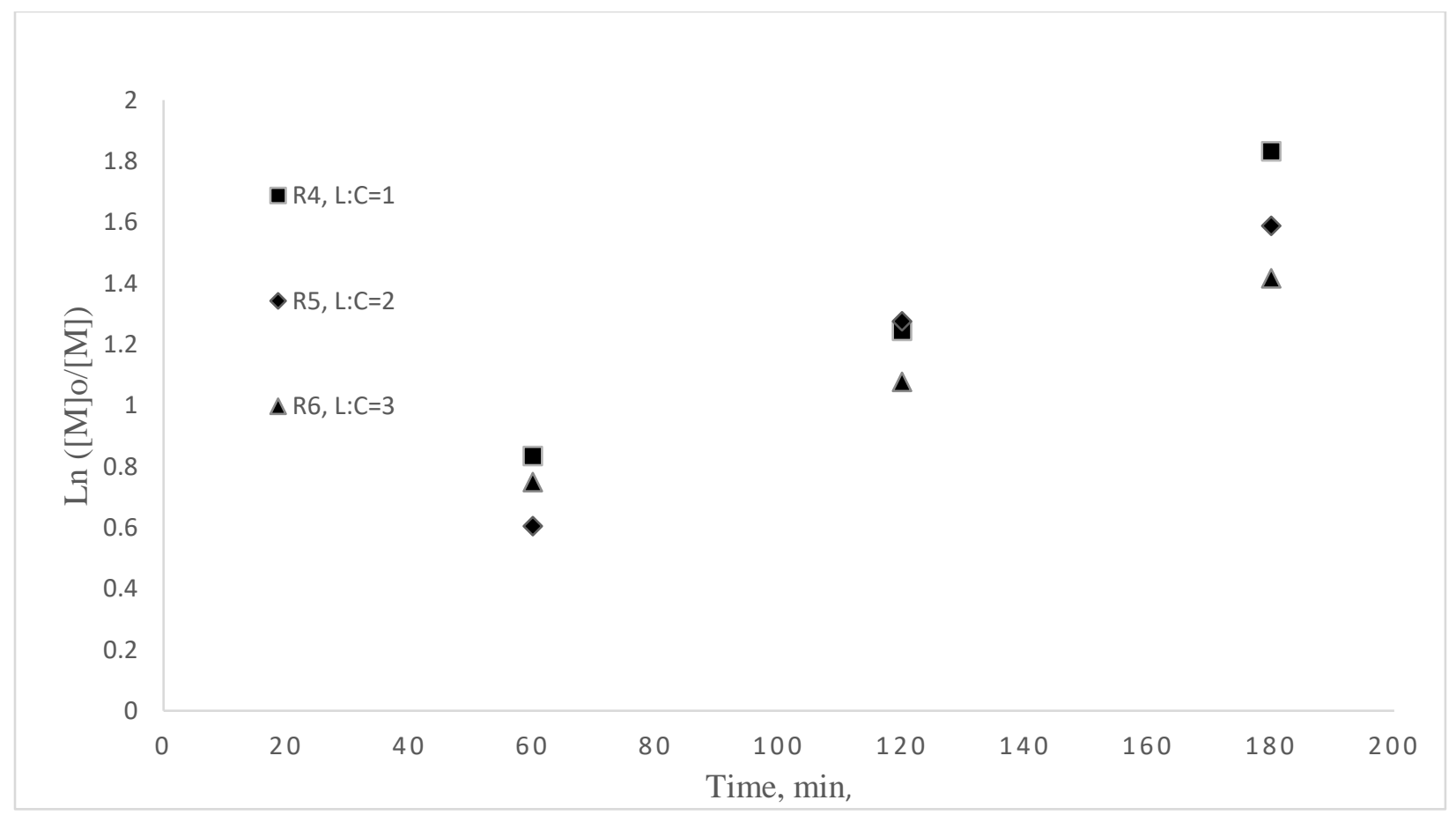

Figure 6.9: First order kinetics plot of BMA versus time for different catalyst/ligand concentration. $[\mathrm{dNbpy}] /\left[\mathrm{CuBr}_{2}\right]=1,1.5$ and 3 respectively. [BMA]:[EBiB]:[CuBr 2$]:[\mathrm{AA}]:[$ Brij98]=213:1:0.12:0.17:5.15, Reaction temp $=70^{\circ} \mathrm{C}$. 


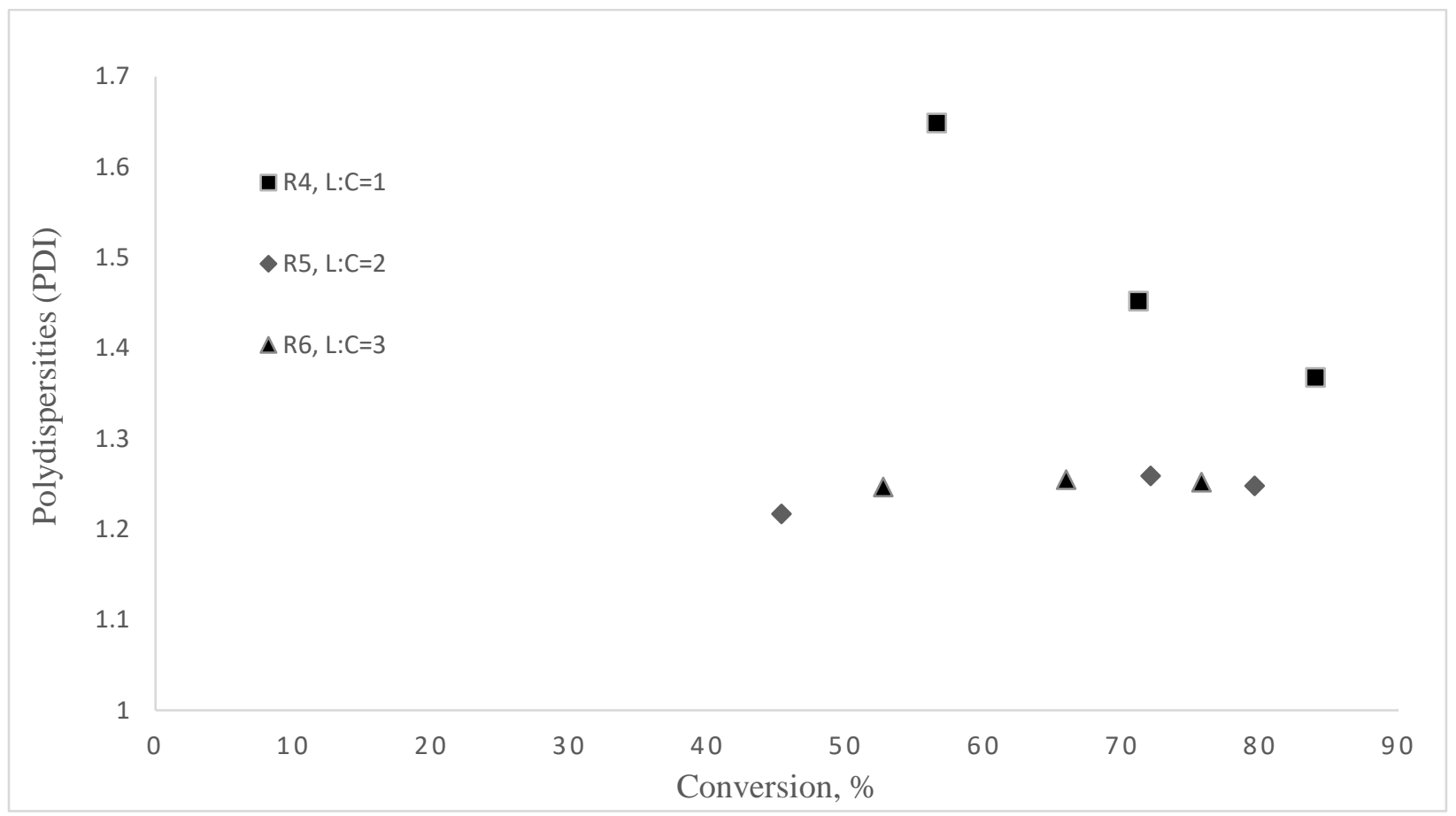

Figure 6.10: PDI versus conversion plot for different reaction catalyst/ligand concentration. $[\mathrm{dNbpy}] /\left[\mathrm{CuBr}_{2}\right]=1$,

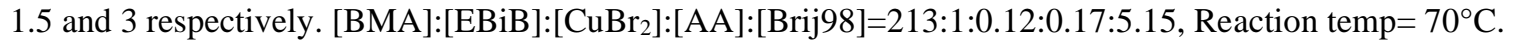

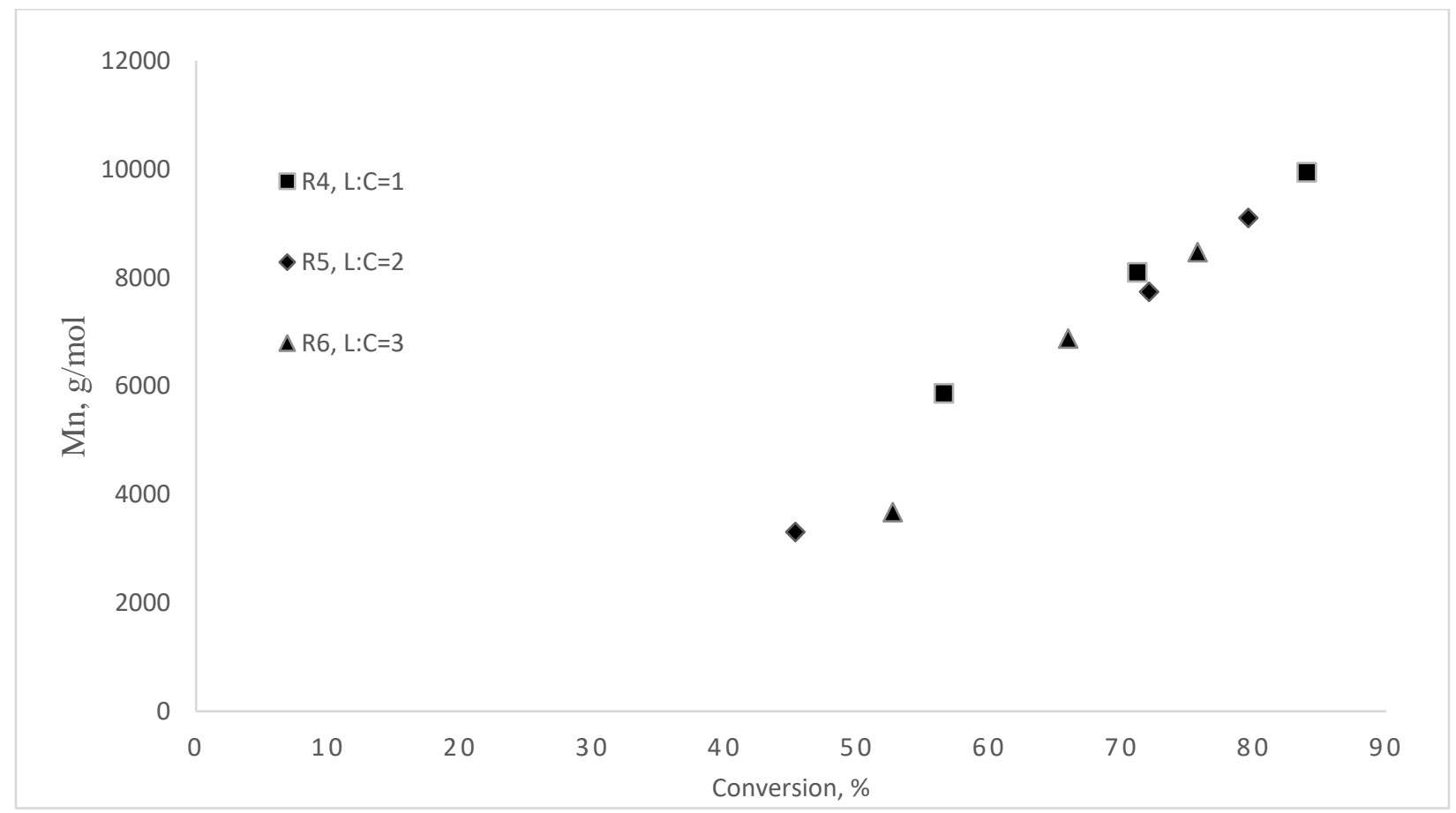

Figure 6.11: Number average molecular weight vs conversion for different catalyst/ligand concentration. $[\mathrm{dNbpy}] /\left[\mathrm{CuBr}_{2}\right]=1,1.5$ and 3 respectively. [BMA]:[EBiB]:[CuBr 2$]:[\mathrm{AA}]:[\mathrm{Brij} 98]=213: 1: 0.12: 0.17: 5.15$, Reaction temp $=70^{\circ} \mathrm{C}$. 
Figure 6.11 illustrates a linear trend of Mn vs BMA conversion. The low Mn of 10450, 9097 and $8472 \mathrm{~g} / \mathrm{mol}$ were obtained as the ligand/catalyst molar ratios varied from 1 to $3 \mathrm{~mol} \mathrm{dNbpy} / \mathrm{mol}$ of $\mathrm{CuBr}_{2}$. The maximum conversion reached is $83.9 \%$ within $3 \mathrm{~h}$ for experimental run $\mathrm{R} 4$ with the smallest ratio of $\mathrm{dNbpy}$ to $\mathrm{CuBr}_{2}$ demonstrating controlled feature of the BMA polymerizationin emulsion system.

An increased of ligand of the same amount of catalyst slowed down the polymerization rate and only lower conversion was obtained in $3 \mathrm{~h}$. However, the 3:1 molar ratio (R6) produces the narrowest MWD with a PDI of 1.119. Results show that a large amount of dNbpy can hamper the metal catalyst effectiveness due to significant increased activity on each $\mathrm{Cu}$ complex, which results to lower monomer conversion at R6 (75.7\%).

\subsection{Surfactant}

Surfactants are among the most important ingredients for monomer polymerization in a dispersed media. The major surfactant functionality is to ensure colloidal stability of latex particles. However, surfactant also serve as monomer droplets stabilizer and may become particle nucleation sites in emulsion polymerization. As a result, a surfactant can affect polymerization kinetics due to the regulation of the number of latex particles and can also act as a chain transfer agent or even a retarder. In a controlled radical polymerization, interaction between the surfactant and radical mediator may also occur and result in loss of polymerization control (Peng et al., 2003; Chan-Seng and Georges, 2006; Wei et al., 2014; Li and Matyjaszewski, 2011).

A surfactant that is suitable for an ATRP system should meet the following criteria: (i) it must not affect the equilibrium between the activation and deactivation processes and (ii) must have a chemical potential to stabilize the dispersed system with minimum amount of coagulum throughout the polymerization medium. Copper-mediated ATRP of BMA in a waterborne system was previously investigated to reveal the impact of various surfactants (Inoue and Matyjaszewski, 2004). Past studies reported that polymerization of BMA was successful in ensuring both colloidal stability and control polymer molecular weights and polydispersity when employing a non-ionic surfactant such as Brij98 (Peng et al., 2003; Oh, 2008). In this study, effect of Brij98 on AGET ATRP of BMA was thoroughly examined. Three experimental tests were performed for different 
surfactant amounts as shown in Table 6.5. In a similar manner to the previous experimental sets the initial amounts of all reactants were kept invariant, except the reagent of interest which is the surfactant Brij98 in this case. Similar to previous experiment procedure, BMA polymerization was done under 20-psig pressure of nitrogen gas and a stirring rate of $250 \mathrm{rpm}$. Also, the second amount of BMA was added after the first 12 min of reaction and nitrogen purging was done for only $5 \mathrm{~min}$. Both the monomer and the reducing agent were halved and added into the reactor according to the two-step experimental procedure.

Table 6.5: AGET ATRP emulsion polymerization using two-step technique for different surfactant concentration.

\begin{tabular}{|r|c|c|c|c|c|c|c|r|r|}
\hline Exp\# & $\begin{array}{c}\text { BMA } \\
\mathrm{I}(\mathrm{g})\end{array}$ & $\begin{array}{c}\mathrm{CuBr}_{2} \\
(\mathrm{~g})\end{array}$ & $\begin{array}{c}\text { dNbpy } \\
(\mathrm{g})\end{array}$ & $\begin{array}{c}\mathrm{H}_{2} \mathrm{O} \\
(\mathrm{g})\end{array}$ & $\begin{array}{c}\text { Brij 98 } \\
(\mathrm{g})\end{array}$ & $\begin{array}{c}\text { EBiB } \\
(\mathrm{g})\end{array}$ & $\begin{array}{c}\mathrm{AA} \\
(\mathrm{g})\end{array}$ & $\begin{array}{c}\text { BMA 2 } \\
(\mathrm{g})\end{array}$ & $\begin{array}{c}\text { Brij 98 } \\
/ \mathrm{H}_{2} \mathrm{O} \text { wt.\% }\end{array}$ \\
\hline R7 & 8.94 & 0.0422 & 0.1508 & 350 & $\mathbf{1 3 . 3 2 8 9}$ & 0.3538 & 0.0507 & 44.5 & 3.81 \\
\hline R8 & 8.94 & 0.0442 & 0.1526 & 350 & $\mathbf{1 0 . 2 9 7 5}$ & 0.3420 & 0.0510 & 44.5 & 2.94 \\
\hline R9 & 8.94 & 0.0430 & 0.1513 & 350 & $\mathbf{7 . 3 3 0 8}$ & 0.3465 & 0.0519 & 44.5 & 2.09 \\
\hline
\end{tabular}

Table 6.6: Experimental result of PBMA by a Two-step emulsion AGET ATRP using different surfactant concentration.

\begin{tabular}{ccccc}
\hline Exp & Time $(\mathrm{min})$ & Conversion $(\%)$ & GPC, Mn $(\mathrm{g} / \mathrm{mol})$ & PDI \\
\hline R7 & 60 & 51.8 & 3624 & 1.432 \\
& 120 & 64.2 & 6931 & 1.340 \\
R8 & 180 & 74.2 & 8188 & 1.375 \\
& 60 & 48.2 & 4053 & 1.376 \\
& 120 & 60.1 & 5800 & 1.313 \\
R9 & 180 & 73.5 & 9140 & 1.308 \\
& 60 & 57.1 & 3980 & 1.306 \\
& 120 & 70.1 & 6414 & 1.275 \\
& 180 & 84.3 & 9045 & 1.279
\end{tabular}


Table 6.6 showed that the PDI decreased in reaction tests which indicated that the polymer chains became uniform as the amount of Brij 98 decreased from about $13.33 \mathrm{~g}$ to $7.33 \mathrm{~g}$. As a result, chain transfer reactions became less significant. Monomer conversion is almost identical in R7 and R8 but increased to $84.3 \%$ in the last test R9 in which the distribution of the polymer chains has small polydispersity (Figure 6.12), indicating steady growth of the polymer chains.

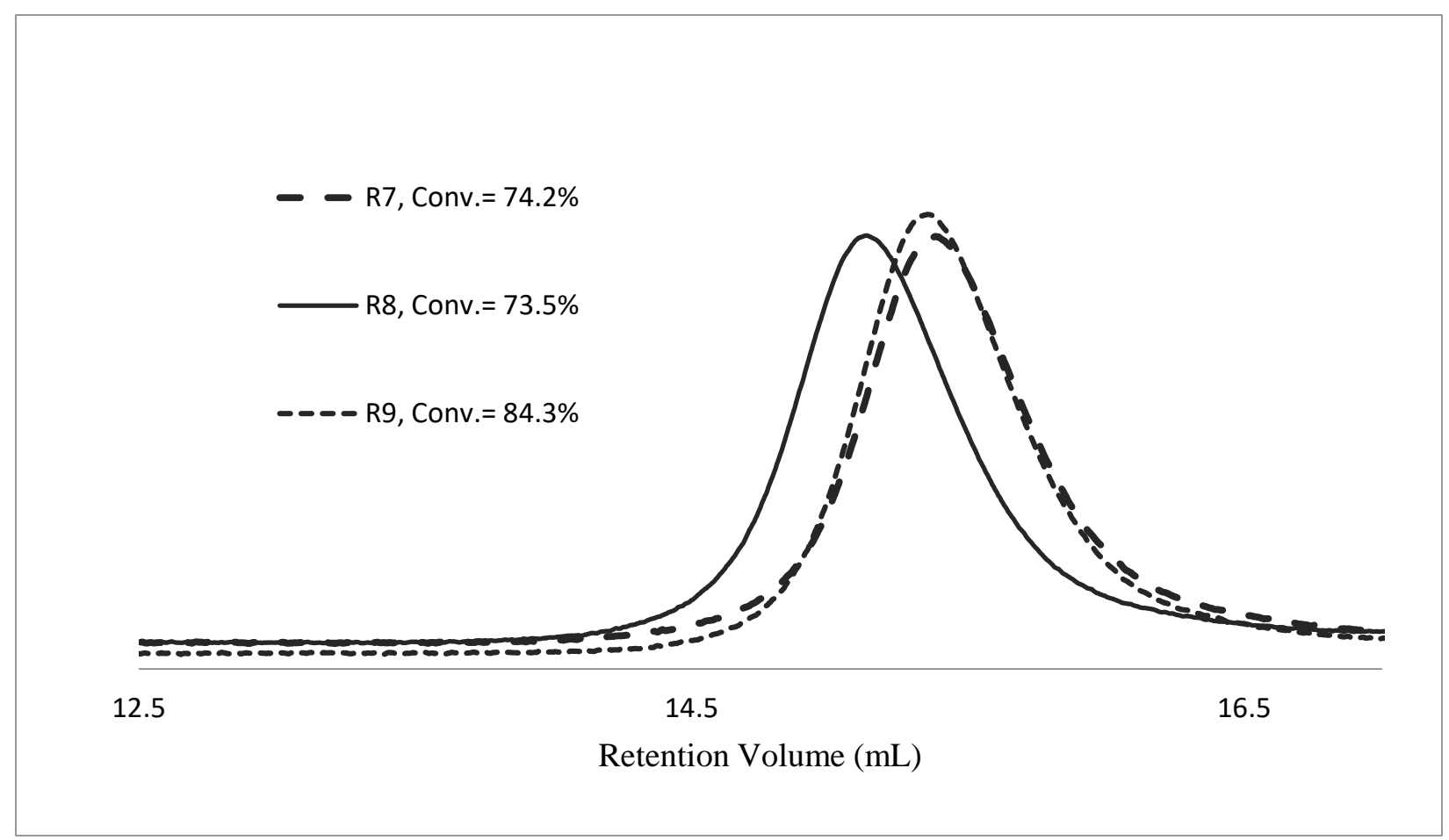

Figure 6.12: GPC traces of the effect of surfactant on BMA polymerization at $180 \mathrm{~min}$. $\mathrm{Brij} 98 / \mathrm{H}_{2} \mathrm{O}=3.81,2.94$ and

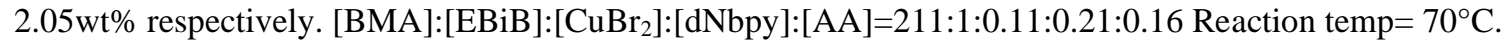

Figure 6.13 showed the BMA conversion versus reaction time. The conversion variation is strikingly linear in all three trials. This demonstrates an unusual monomer conversion versus time. The addition of more surfactant did not modify the conversion linear trend but improved the BMA conversion. Figure 6.14 showed the expected first order plot for all the system with high initial conversion. Initially, the low surfactant ratio (R9) showed higher monomer conversion of $57.1 \%$ at time $1 \mathrm{~h}$, than the higher surfactant loads R7 and R8 with $51.8 \%$ and $48.2 \%$ respectively. 


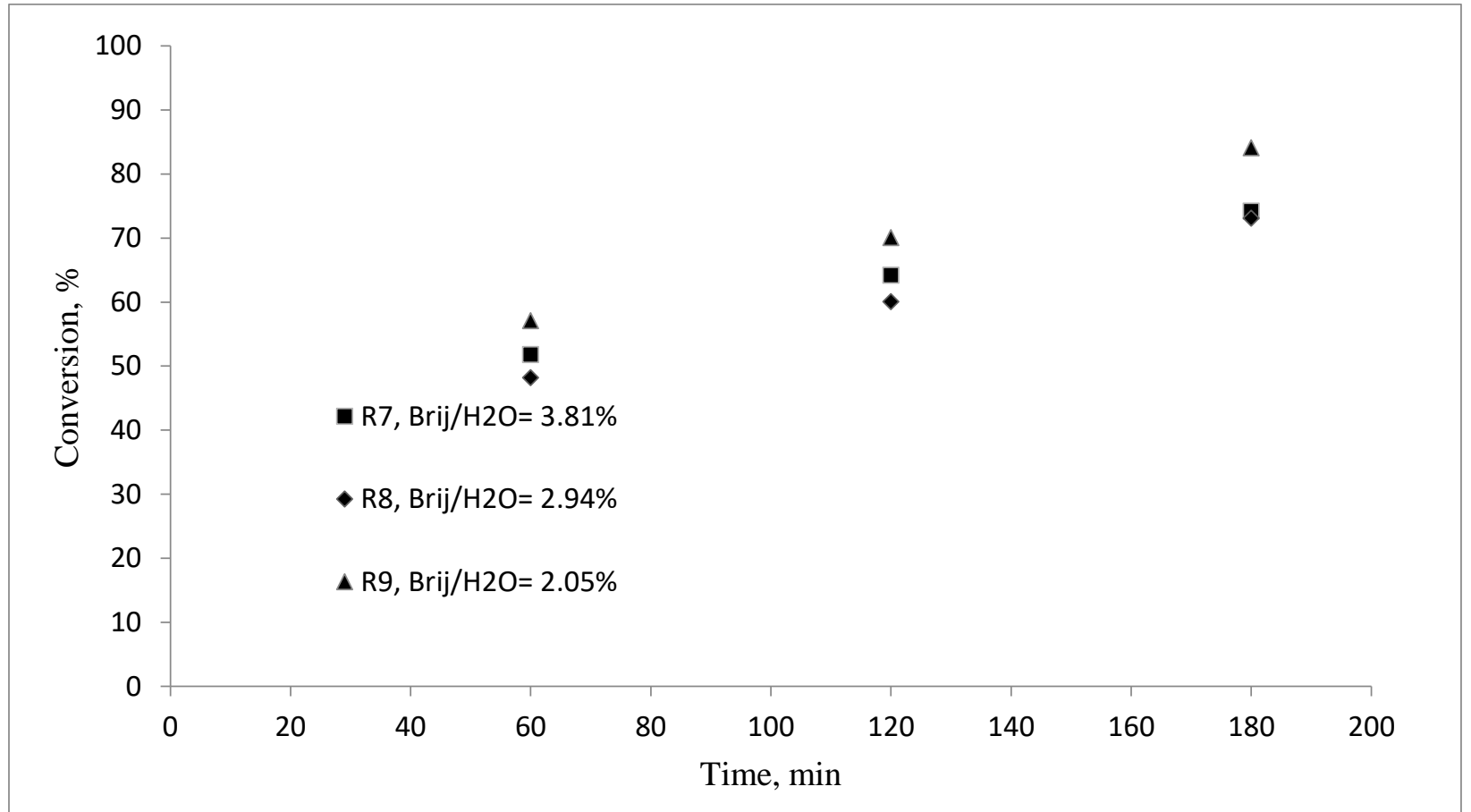

Figure 6.13: Conversion versus time for different surfactant concentration. Brij98/ $\mathrm{H}_{2} \mathrm{O}=3.81,2.94$ and 2.05wt $\%$ respectively. [BMA]:[EBiB]:[CuBr 2$]:[\mathrm{dNbpy}]:[\mathrm{AA}]=211: 1: 0.11: 0.21: 0.16$ Reaction temp $=70^{\circ} \mathrm{C}$.

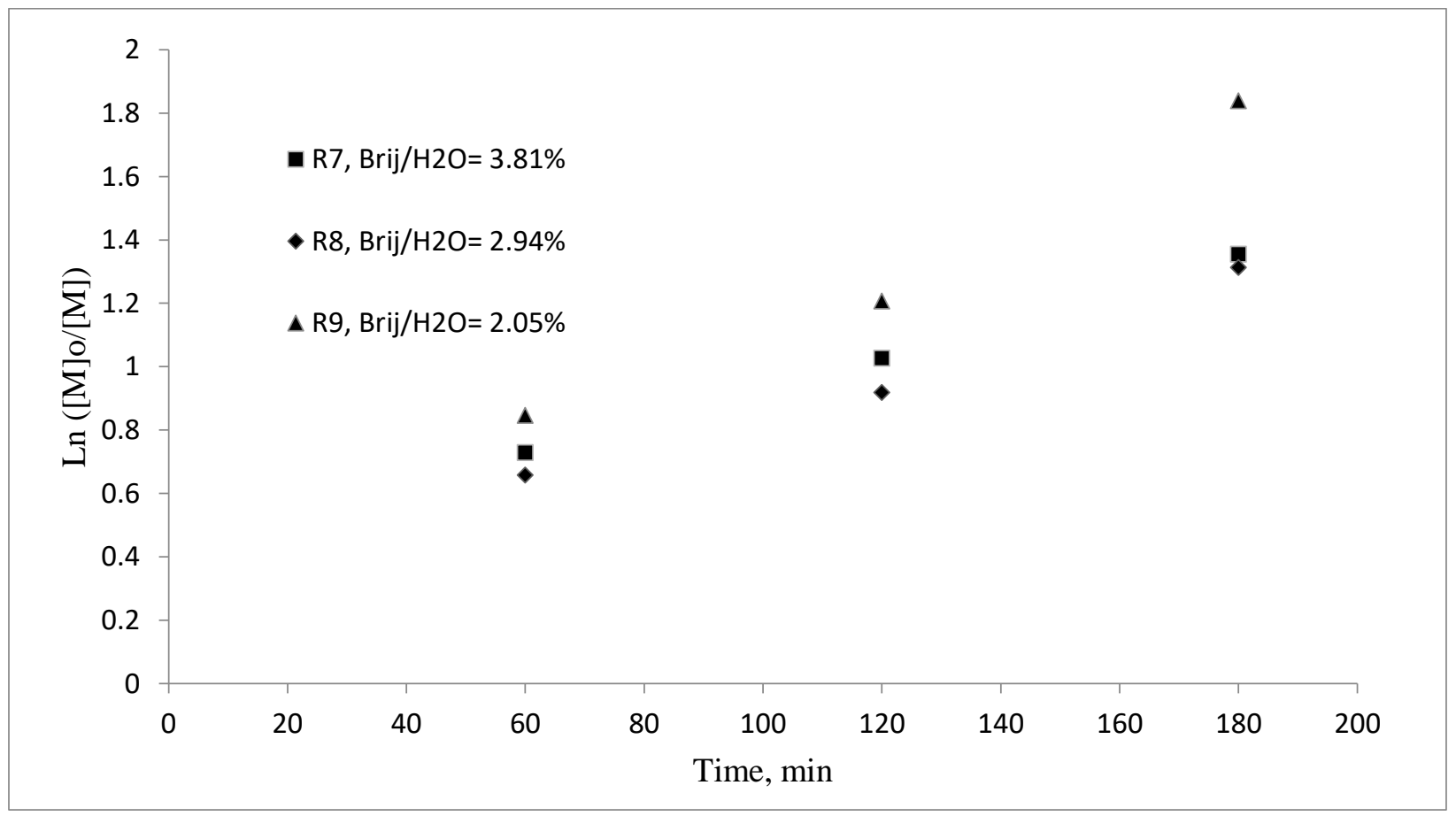

Figure 6.14: First order kinetics plot of BMA versus time for different surfactant concentration. $\mathrm{Brij} 98 / \mathrm{H}_{2} \mathrm{O}=3.81$, 2.94 and $2.05 \mathrm{wt} \%$ respectively. [BMA]:[EBiB]:[CuBr 2$]:[\mathrm{dNbpy}]:[\mathrm{AA}]=211: 1: 0.11: 0.21: 0.16$ Reaction temp $=70^{\circ} \mathrm{C}$. 
Figure 6.15 shows that experiments R7 and R8 display narrow PDI values of 1.375 and 1.308 and low average MWs of $8188 \mathrm{~g} / \mathrm{mol}$ and $9140 \mathrm{~g} / \mathrm{mol}$ at similar conversions of $74.2 \%$ and $73.5 \%$, indicating a controlled polymerization. In addition, as the surfactant amount increased to higher than 2 wt $\%$ ratio with $\mathrm{H}_{2} \mathrm{O}$, no substantial effect was observed on the system. Peng et al., (2005) obtained similar results in running BMA ATRP emulsion system. In experiment R9, a narrow PDI of 1.279 and low average molecular weight of $9045 \mathrm{~g} / \mathrm{mol}$ with $84.3 \mathrm{wt} \%$ conversion was obtained. However, some traces of precipitation were found on the impeller with $2.09 \mathrm{wt} \%$ of the surfactant (R9). Thus, increasing more surfactant showed better stability of the emulsion pattern, with almost no coagulum observed on R7 and R8. Figure 6.16 illustrates the linear trend of Mn vs BMA conversion. The low Mn of 8188, 9140 and $9045 \mathrm{~g} / \mathrm{mol}$ were obtained as ratio of surfactant with $\mathrm{H}_{2} \mathrm{O}$ decreases.

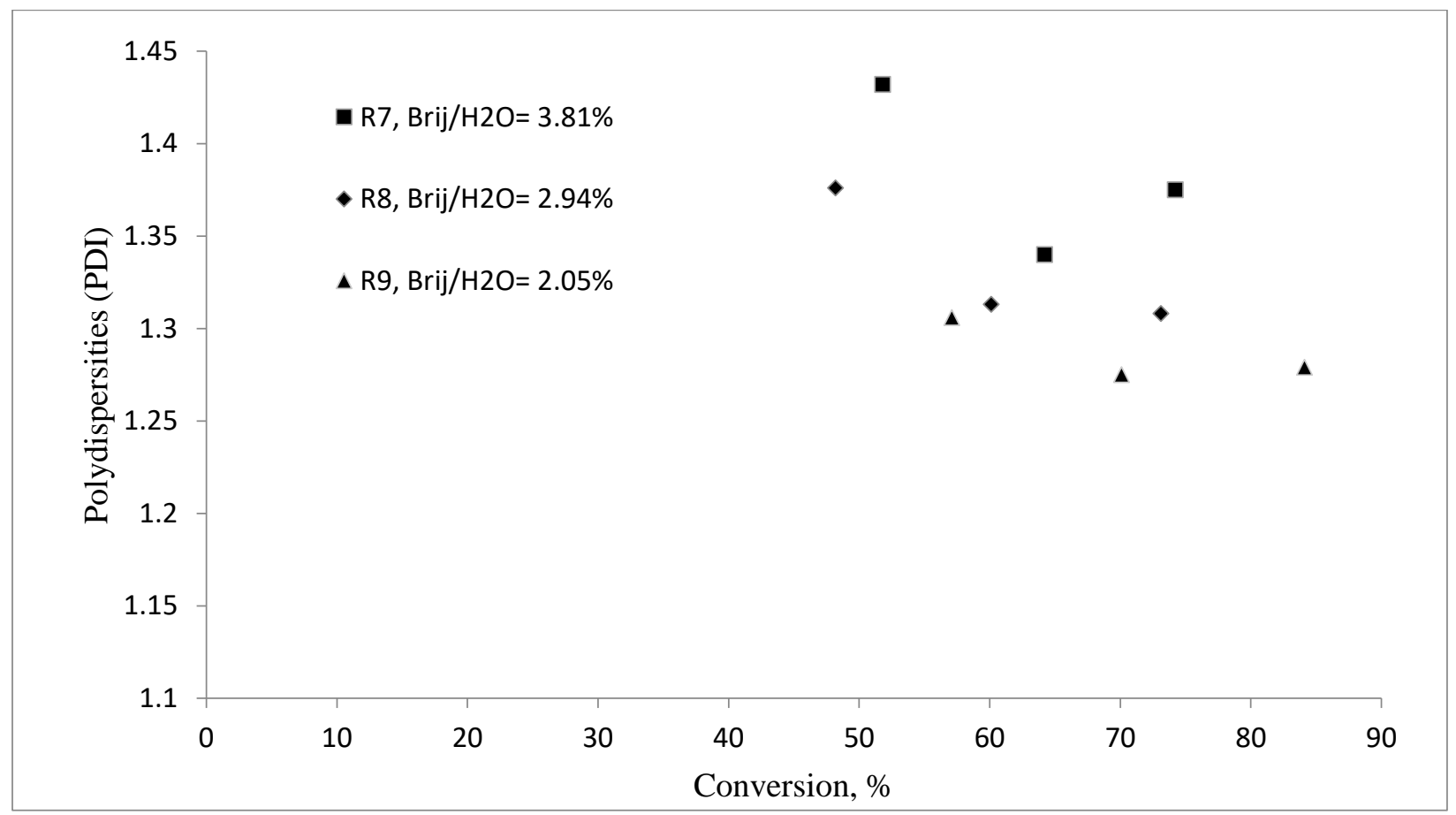

Figure 6.15: PDI versus conversion plot for different surfactant concentration. $\mathrm{Brij} 98 / \mathrm{H}_{2} \mathrm{O}=3.81,2.94$ and 2.05 wt $\%$ respectively. [BMA]:[EBiB]:[CuBr 2$]:[\mathrm{dNbpy}]:[\mathrm{AA}]=211: 1: 0.11: 0.21: 0.16$ Reaction temp $=70^{\circ} \mathrm{C}$. 


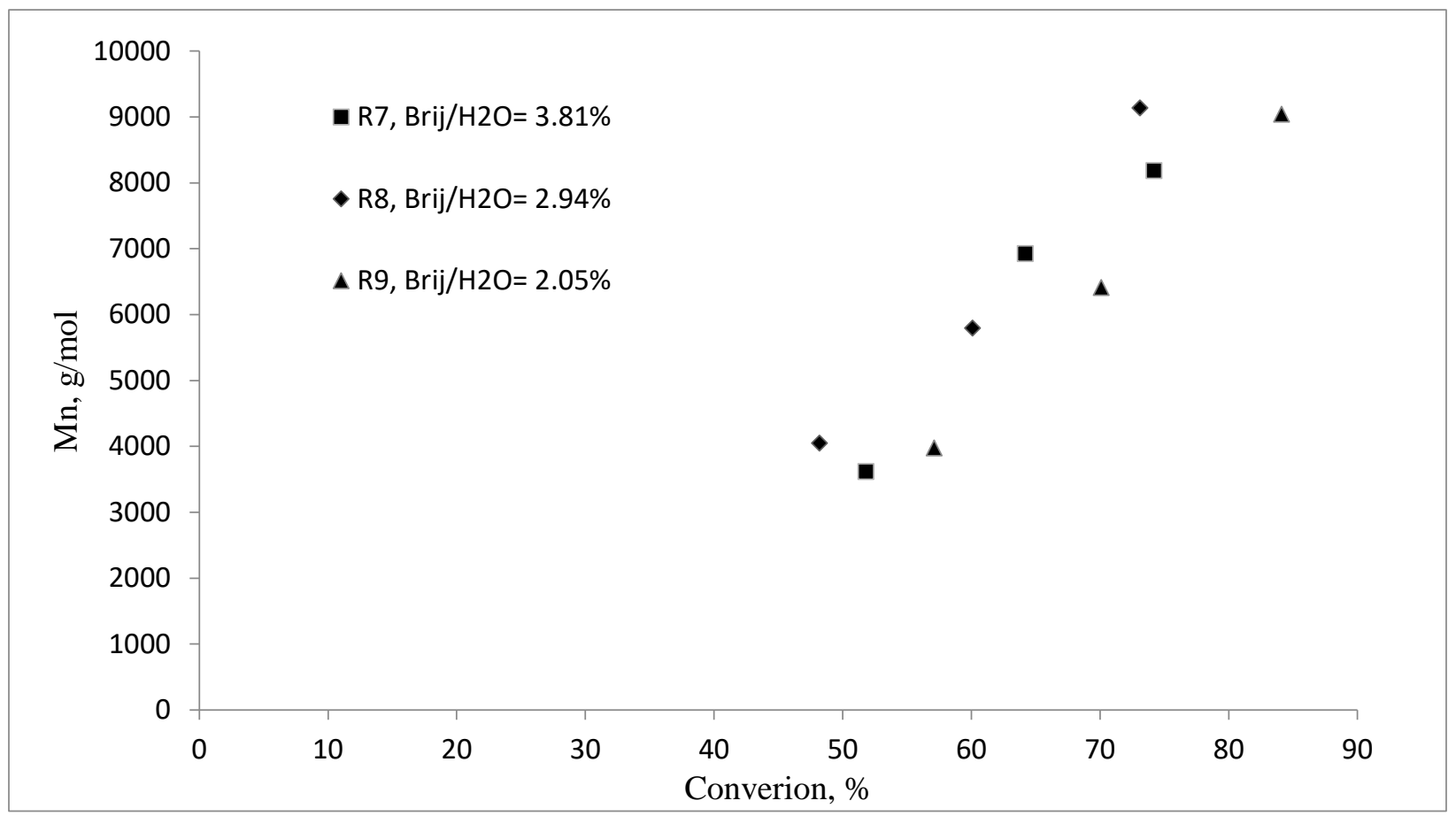

Figure 6.16: Number average molecular weight vs conversion for different surfactant concentration. $B$ rij $98 / \mathrm{H}_{2} \mathrm{O}=$ 3.81, 2.94 and 2.05wt\% respectively. [BMA]:[EBiB]:[CuBr 2$]:[\mathrm{dNbpy}]:[\mathrm{AA}]=211: 1: 0.11: 0.21: 0.16$ Reaction temp= $70^{\circ} \mathrm{C}$.

Hence, BMA polymerizations with the non-ionic surfactant Brij 98 were colloidally stable and chemically well controlled according to the reaction principles adopted in CRP. Polymers of low MW and low polydispersity were produced. Also, variability in latex colloidal stability was somewhat revealed. In lower surfactant/water ratio, traces of solid residue were observed at the end of the polymerization process.

\subsection{Reducing Agent}

Ascorbic acid was selected as the reducing agent in this study. It is a strong reducing agent and can quickly reduce $\mathrm{Cu}$ (II) to $\mathrm{Cu}$ (I). Generally, AGET ATRP can accept a relatively large range of reducing agent concentrations. However, to achieve a controlled polymerization, selecting the appropriate amount of reducing agent is a key procedure (Min et al., 2005). Small amounts of ascorbic acid may lead to a slow polymerization, whereas large amounts would lead to a reduced level of control. It is also important to note that ascorbic acid is a two-electron reducing agent. In this experiment, three [Cu (II)]/[Ascorbic Acid] ratios were analyzed as shown in Table 6.7. The 
initial amounts of all reactants were kept invariant, except for the initial AA amount. Three BMA polymerization runs were carried out under 20-psig pressure of nitrogen gas, the medium was stirred at $250 \mathrm{rpm}$ and the second amount of BMA was added after the first 12 min of reaction. Nitrogen purging was done during the microemulsion polymerization for only $5 \mathrm{~min}$. The amount of AA was split into two equal halves. The second half of AA was dissolved in $10 \mathrm{ml}$ of distilled water and then was added immediately after the addition of BMA.

Table 6.7: AGET ATRP emulsion polymerization using two-step technique for different reducing agent concentration.

\begin{tabular}{|c|r|r|r|r|r|r|r|r|r|}
\hline Run \# & $\begin{array}{c}\text { BMA I } \\
(\mathrm{g})\end{array}$ & $\begin{array}{c}\mathrm{CuBr}_{2} \\
(\mathrm{~g})\end{array}$ & $\begin{array}{c}\mathrm{dNbpy} \\
(\mathrm{g})\end{array}$ & $\begin{array}{c}\mathrm{H}_{2} \mathrm{O} \\
(\mathrm{g})\end{array}$ & $\begin{array}{c}\text { Brig 98 } \\
(\mathrm{g})\end{array}$ & $\begin{array}{c}\text { EBiB } \\
(\mathrm{g})\end{array}$ & $\mathbf{A A}(\mathrm{g})$ & $\begin{array}{c}\text { BMA 2 } \\
(\mathrm{g})\end{array}$ & $\begin{array}{c}{\left[\mathrm{CuBr}_{2}\right]:} \\
{[\mathrm{AA}]}\end{array}$ \\
\hline $\mathrm{R} 10$ & 8.94 & 0.0463 & 0.1559 & 350 & 10.2667 & 0.3533 & $\mathbf{0 . 1 0 1 6}$ & 44.5 & $1: 3.0$ \\
\hline $\mathrm{R} 11$ & 8.94 & 0.0430 & 0.1513 & 350 & 10.2353 & 0.3578 & $\mathbf{0 . 0 5 2 3}$ & 44.5 & $1: 1.5$ \\
\hline $\mathrm{R} 12$ & 8.94 & 0.0433 & 0.1512 & 350 & 10.2465 & 0.3682 & $\mathbf{0 . 0 3 1 4}$ & 44.5 & $1: 1.0$ \\
\hline
\end{tabular}


Table 6.8: Experimental result of PBMA by a Two-step emulsion AGET ATRP of BMA using different reducing agent concentration.

\begin{tabular}{ccccc}
\hline Exp & T $(\min )$ & Conv $(\%)$ & Mn, GPC $(\mathrm{g} / \mathrm{mol})$ & PDI \\
\hline R10 & 60 & 60.9 & 6839 & 1.16 \\
& 120 & 86.6 & 10128 & 1.25 \\
R11 & 180 & 90.2 & 10325 & 1.29 \\
& 60 & 45.3 & 3305 & 1.22 \\
R12 & 120 & 75.2 & 7980 & 1.26 \\
& 180 & 84.9 & 9185 & 1.31 \\
& 60 & 48.6 & 4492 & 1.46 \\
& 120 & 53.9 & 4703 & 1.22 \\
& 180 & 60.9 & 5289 & 1.15
\end{tabular}

Table 6.8 shows the gravimetry and GPC analysis. Figure 6.17 shows the GPC traces for experiments R10, R11 and R12, which illustrates that the polymer chain distribution has small polydispersity. In addition, high initial conversion of $45.3 \%, 60.9 \%$ and $48.6 \%$ was observed in all three reactions as shown in Figure 6.18. A slow polymerization rate was then observed with the 1.0 ratio of $\left[\mathrm{CuBr}_{2}\right] /$ [Ascorbic Acid] for trial (R12) as shown from the linear first order kinetics plot (Figure 6.19). Experiment R12 displayed a very narrow PDI of (1.15) and low average molecular weights of $(5289 \mathrm{~g} / \mathrm{mol})$ despite a low conversion of $(60.9 \%)$ after $3 \mathrm{~h}$. The trials confirm a well-controlled polymerization although the monomer conversion did not exceed $61 \%$. However, the small amount of ascorbic acid in the system led to a slower polymerization in comparison to trials, R10 and R11 (1.5 and 3.0). 


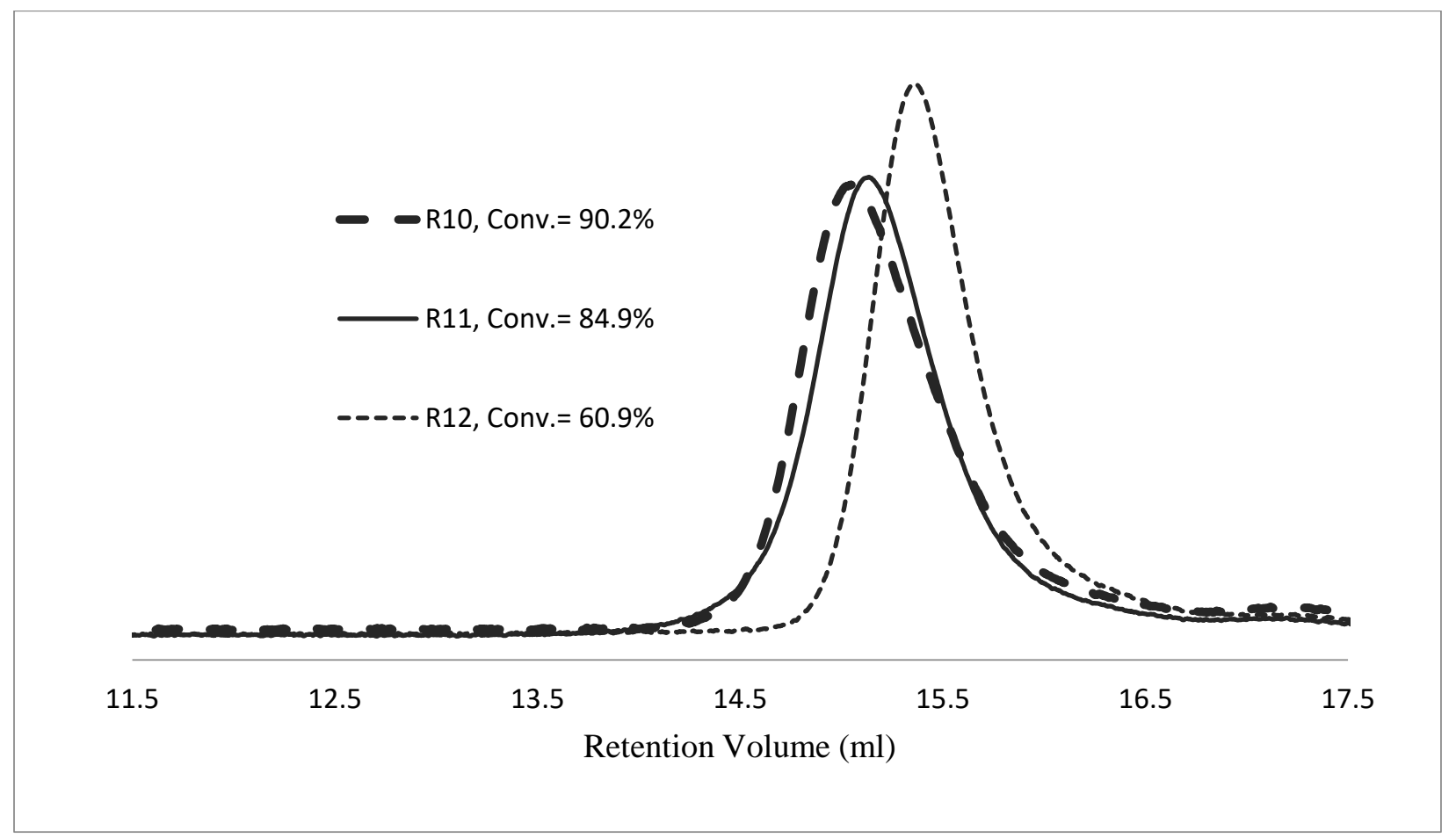

Figure 6.17: GPC traces of the effect of reducing agent on BMA polymerization at $180 \mathrm{~min}$. [Cu(II)]/[AA] $=0.33$, 0.67 and 1 respectively. [BMA]:[EBiB]: $\left[\mathrm{CuBr}_{2}\right]:[\mathrm{dNbpy}]:\left[\right.$ Brij98] $=205: 1: 0.10: 0.20: 4.87$, Reaction temp $=70^{\circ} \mathrm{C}$.

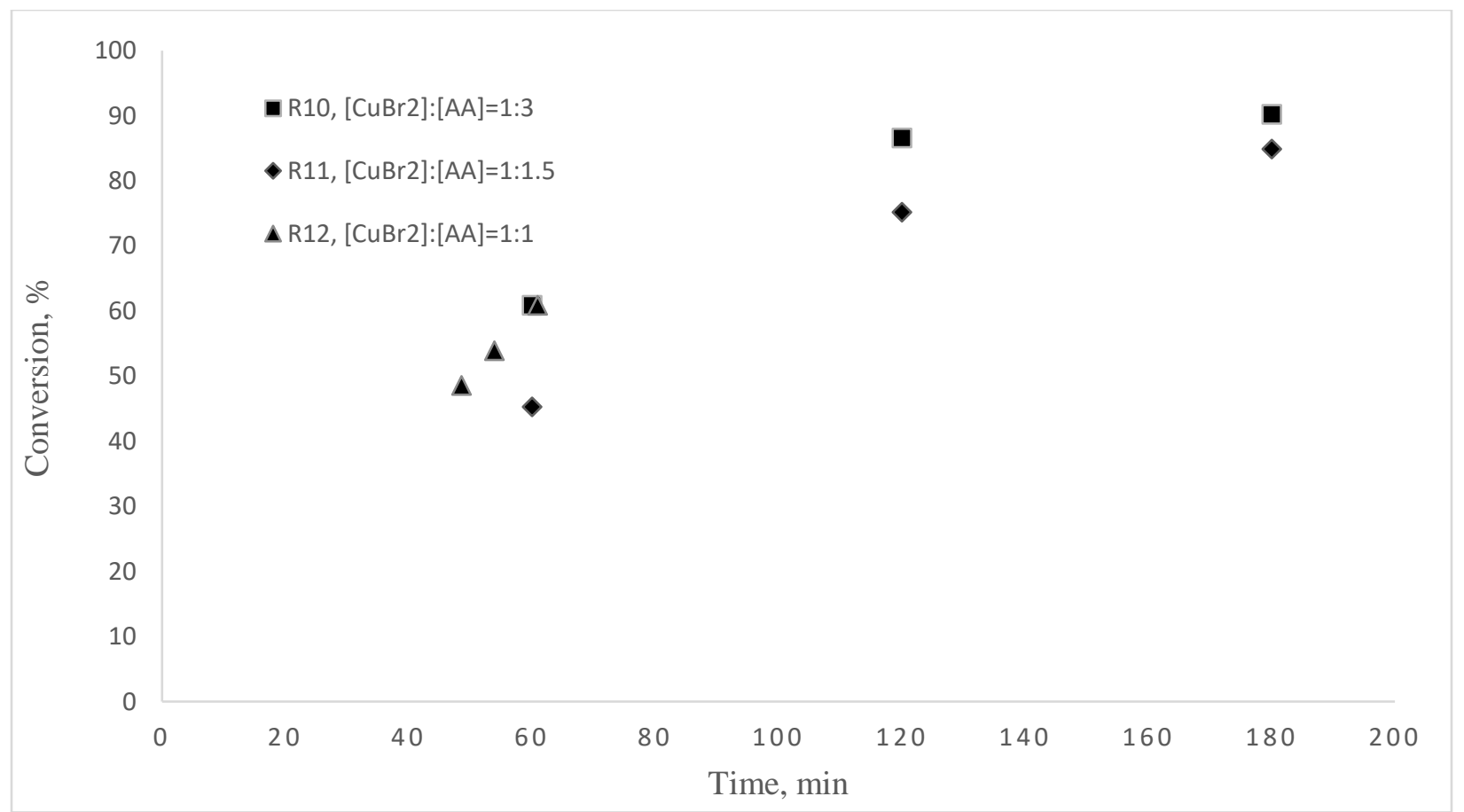

Figure 6.18: Conversion versus time for different reducing agent concentration.[Cu(II)]/[AA] $=033,0.67$ and 1 respectively. [BMA]:[EBiB]:[CuBr 2$]:[\mathrm{dNbpy}]:[\mathrm{Brij} 98]=205: 1: 0.10: 0.20: 4.87$, Reaction temp $=70^{\circ} \mathrm{C}$. 


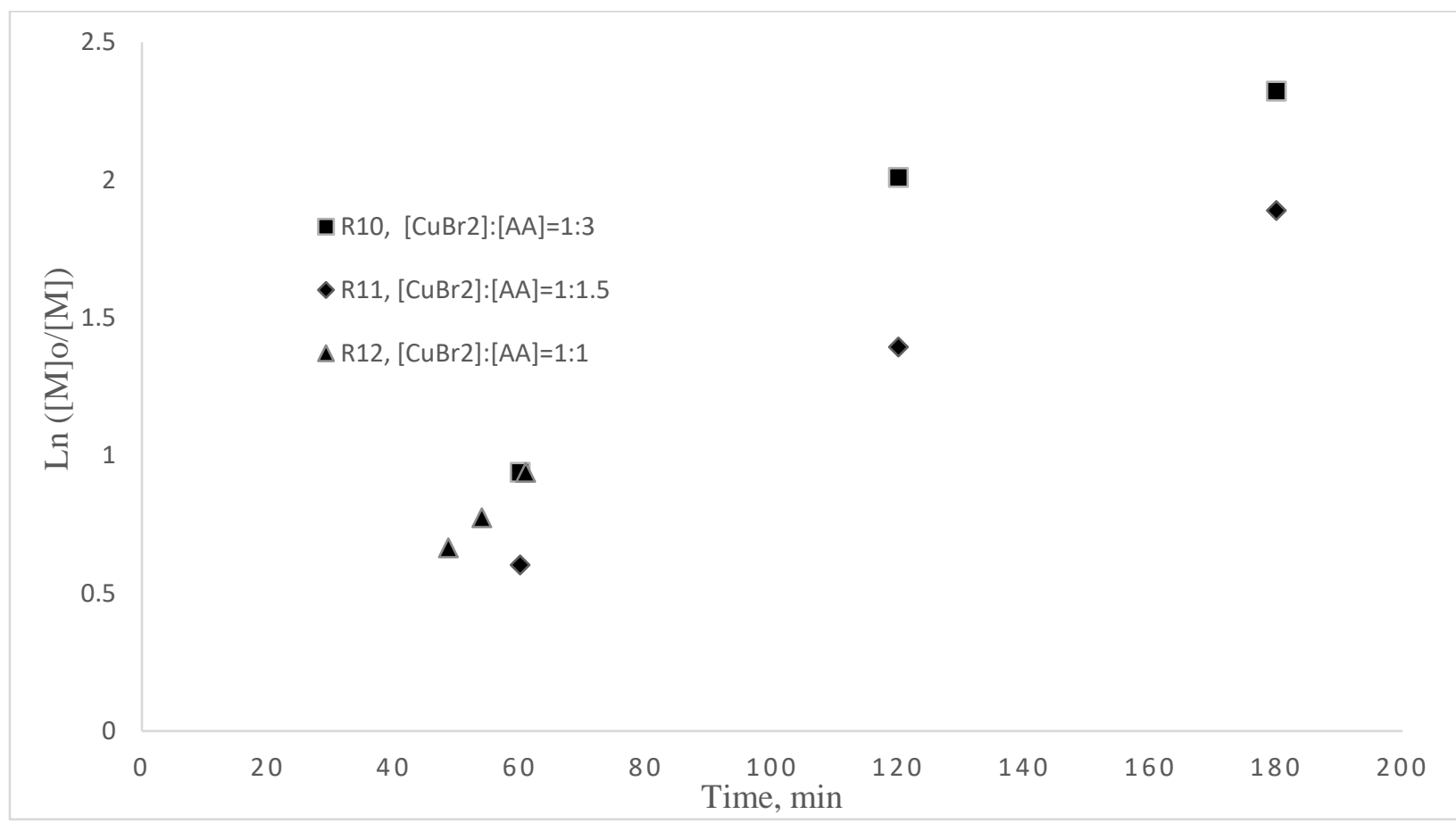

Figure 6.19: First order kinetics plot of BMA versus time for different reducing agent concentration. $[\mathrm{Cu}(\mathrm{II})] /[\mathrm{AA}]=0.33,0.67$ and 1 respectively. [BMA]:[EBiB]:[CuBr 2$]:[\mathrm{dNbpy}]:[\mathrm{Brij} 98]=205: 1: 0.10: 0.20: 4.87$, Reaction temp $=70^{\circ}$.

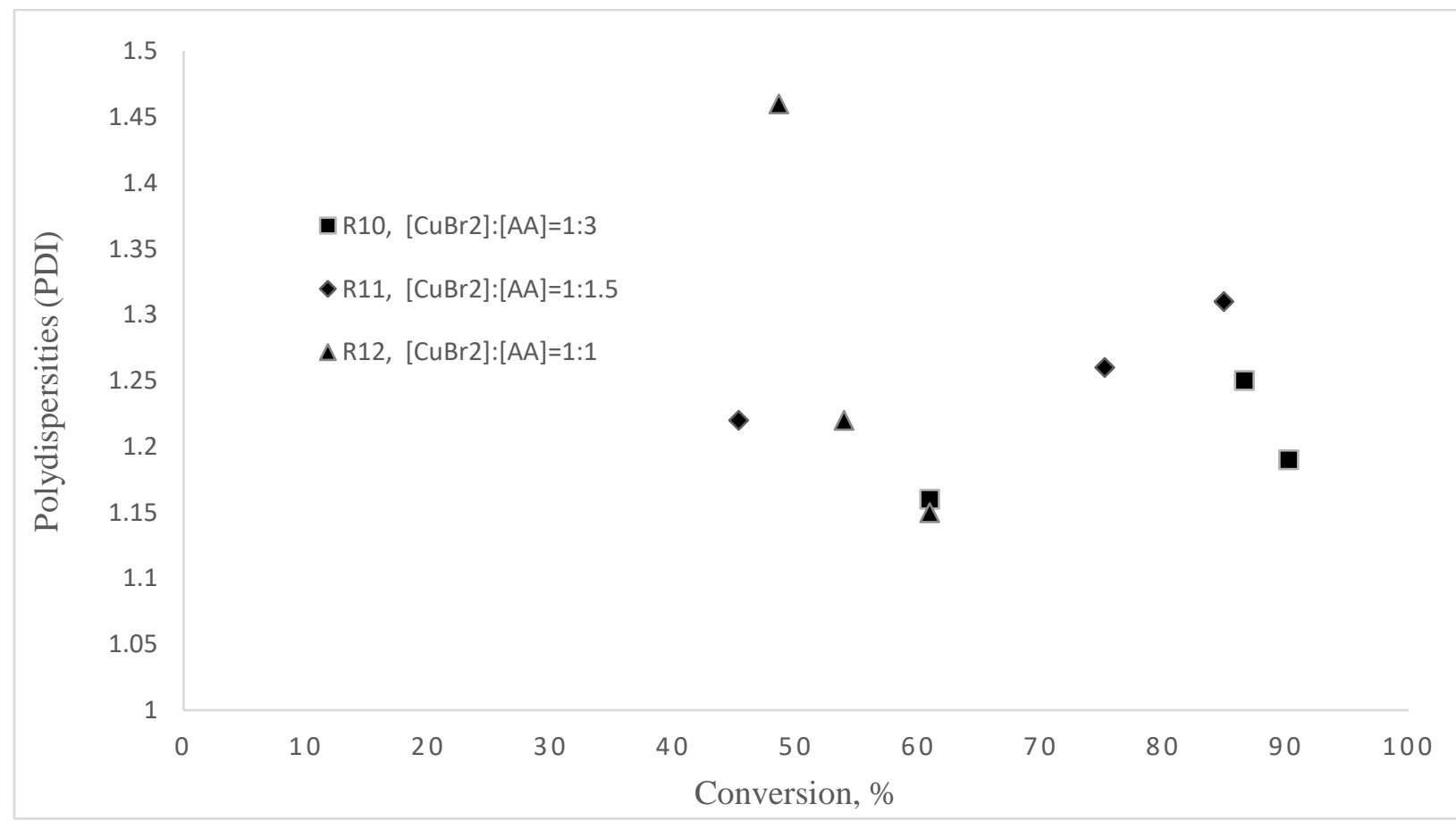

Figure 6.20: $P D I$ versus conversion plot for different reducing agent concentration. $[\mathrm{Cu}(\mathrm{II})] /[\mathrm{AA}]=0.33,0.67$ and 1 respectively. [BMA]:[EBiB]:[$\left[\mathrm{CuBr}_{2}\right]$ :[dNbpy]:[Brij98] $=205: 1: 0.10: 0.20: 4.87$, Reaction temp $=70^{\circ} \mathrm{C}$. 


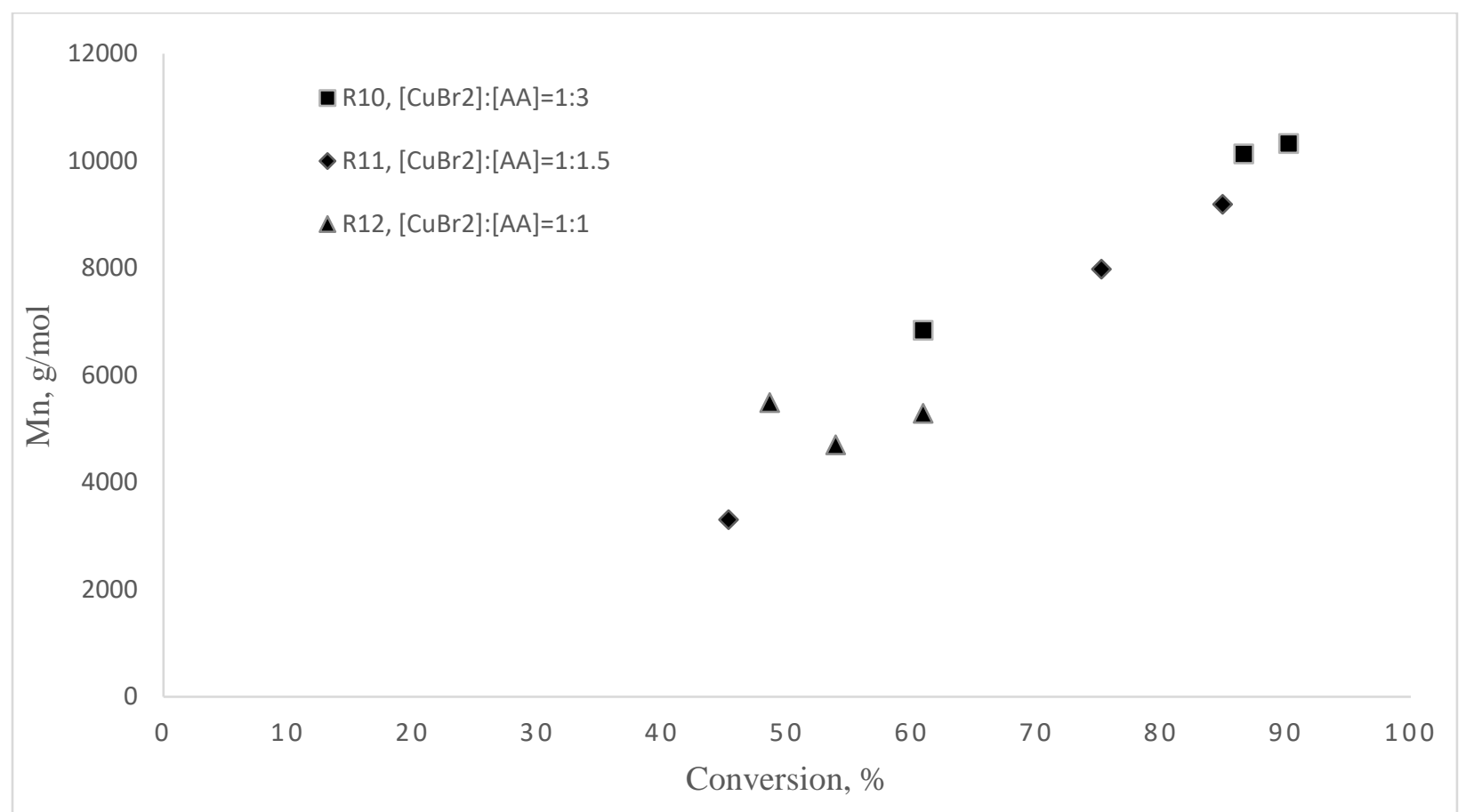

Figure 6.21: Number average molecular weight vs conversion for different reducing agent concentration. $[\mathrm{Cu}(\mathrm{II})] /[\mathrm{AA}]=0.33,0.67$ and 1 respectively. [BMA]:[EBiB]:[CuBr 2$]:[\mathrm{dNbpy}]:[\mathrm{Brij} 98]=205: 1: 0.10: 0.20: 4.87$, Reaction temp $=70^{\circ} \mathrm{C}$.

In experiments R10 and R11, the conversion profile (Figure 6.18) showed a faster polymerization rates compared to R12, but no significant differences were observed between R10 and R11. Both data depicts a narrow PDI values of 1.29 and 1.31 and low average MWs of $10325 \mathrm{~g} / \mathrm{mol}$ and $9185 \mathrm{~g} / \mathrm{mol}$. However, the observed increasing polydispersity with polymerization time as shown in Figure 6.20 indicated the presence of termination in the system.

The Mn-conversion profile (Figure 6.21) showed a better controlled trend at the 1.0 ratio in trial $\mathrm{R} 12$, as evidenced by a low polydispersity. The polymerization conducted at $1.0[\mathrm{Cu}$ (II)]/[ascorbic acid] ratio indicated a better controlled polymerization compared to the other ratios used.

\subsection{Conclusions}

BMA polymerization showed very fast reaction rates, with high initial conversions reached in short time periods, resulting in a small-low-molecular weight tails in the GPC traces. Decreasing temperature can improve polymerization livingness, but very low temperatures may lead to less 
controlled polymer chains. The trials showed that the polymerization rates increased with the [catalyst]: [ligand] ratio and that reducing the copper to ligand concentration ratios led to better control over the polymerization process. A better controlled polymerization was obtained with a 1.0 copper to reducing agent ratio. A $2.0 \mathrm{wt}$. $\%$ of surfactant to water produced stable latexes and reduced coagulation. 


\section{CHAPTER 7}

\section{CONCLUSIONS AND RECOMMENDATIONS}

\subsection{Conclusions}

Most ATRP polymerization using various initiation techniques have been done in bulk and solution media in small ampule reactors. The objective of this thesis was to extend the understanding of AGET ATRP of BMA under emulsion polymerization condition in a larger reactor. Early research attempts on living/controlled radical polymerization under emulsion conditions were surprisingly challenging. This challenge was mainly attributed to the instability of polymer particles due to lack of initial high molecular weight polymer chains at the early stage of polymerization. To overcome this problem a two-step emulsion technique was utilized to get colloidal stable latex.

In this study, ATRP polymerization of butyl methacrylate (BMA) was investigated in a 2-L stirred tank reactor using AGET as the initiation technique. AGET ATRP has been successfully extended to an emulsion medium using a stepwise two-step procedure. Preliminary experimental trials have highlighted the need for a proper choice of surfactant, the use of adequate amounts of a hydrophobic ligand and metal catalyst to prevent an eventual oxidation of the metal catalyst and its partitioning between polymer particles and aqueous phase.

In the two-step procedure, an emulsion was formed by introducing monomer into an ongoing microemulsion system. A small sized nano-structured material with controlled molecular distribution (polydispersity $~ 1.5$ ) were successfully synthesized using the two-step microemulsion process with low surfactant concentration e.g. surfactant/monomer ratio $\sim 1: 3.5$ in an aqueous system. Several factors were shown to significantly influence the AGET ATRP polymerization process and needed to be investigated to ensure a stable and controlled polymerization. Thus, effects of reaction temperature and the initial concentrations of ligand/catalyst, surfactants, and reducing agent on polymerization were investigated. The most important concluding remarks are as follows:

1. BMA polymerization with different ligands: PMDETA, HMTA and dNbpy were first compared. A very slow polymerization occurred in PMDETA, while the polymerization 
was too fast and not controlled when HMTA was used. Thus, the result showed that only the hydrophobic ligand dNbpy proved to be readily applicable.

2. With dNbpy ligand, BMA polymerization showed a very fast reaction rate, and relatively high values of initial monomer conversion were reached in a short time (R1: $37.9 \%$, $\mathrm{R} 2: 45.6 \%, \mathrm{R} 3: 52.7 \%$ ). Increasing the temperature to certain extent improved the livingness of the polymerization (PDI for R1:1.338, R2:1.224, R3:1.265) at temperature $50^{\circ} \mathrm{C}, 60^{\circ} \mathrm{C}$ and $70^{\circ} \mathrm{C}$ respectively.

3. Varying surfactant concentration $(3.81 \mathrm{wt} \%, 2.94 \mathrm{wt} \%$ and $2.0 \mathrm{wt} \%)$ has also similar effects on PDI, conversion and molecular weight. In addition, the surfactant concentration was decreased to $\sim 2 \mathrm{wt} \%$ ratio with $\mathrm{H}_{2} \mathrm{O}$. However, some traces of coagulum were found on the impeller.

4. Increasing the molar ratio of catalyst to ligand (R5>R4>R6) results in high initial polymerization conversion, but the overall conversion in each run were almost the same at the end of polymerization (R5:83.9\%, R4:79.6\%; R6:75.7\%). Thus, large amount of ligand can badly affect the metal catalyst activity.

5. Better control of BMA polymerization was obtained when the reaction temperature was held at $70^{\circ} \mathrm{C}$ and the ratio of ascorbic acid to catalyst was close to unity.

\subsection{Recommendations}

Although the two-step microemulsion/emulsion polymerization process was shown to be a promising technique for producing nanoparticles with low concentration of surfactant, and the performance of BMA polymerization was thoroughly investigated by varying several experimental factors. This study can be regarded as an initial step to promote further the two-step polymerization technique. Further, detailed investigation must be done before the procedure can be applied as a general technique in an industrial scale. Experimental trials that have been tested in this study showed the potentiality of controlled polymerization and pointed out the problems required to be resolved. 
1. The effects of catalyst concentration, surfactants, ligands, temperature and reducing agent concentration on polymerization were investigated only one at a time, and not all factors were simultaneously varied for a given BMA monomer conversion. Factorial design is therefore recommended to investigate the effects of multiple factors on the polymerization and determine the most significant experimental scenario to optimize the system performance.

2. The polymerization system kinetics of AGET ATRP is quite complex. Studying the reactor performance as a black box system cannot reveal a reasonable insight of the reactor behavior. Therefore, a mechanistic model including mass transfer between aqueous and oil phase, equilibrium thermodynamics and the kinetics polymerization should be built to pave the way for a better understanding of the system behavior and improve the reactor performance.

3. The effect of surfactant on colloidal stability should be explored further using different surfactants. 


\section{APPENDIX A:}

\section{A.1 Recent Developments in ATRP}

Different ATRP methods have been developed over the past 25 years including the normal, reverse, SR\&NI, AGET, ARGET and ICAR ATRP which dissimilarities are expressed in terms of the initiation types.

\section{A.2 Normal, Reverse, SR\&NI ATRP}

In a normal ATRP reaction reported (Sawamoto et al., 1995; Matyjaszewski et al., 1995), the initiating radicals are generated from an alkyl halide in presence of relatively high concentration of a transition metal catalyst in its lower oxidation state (e.g. $\left.\mathrm{CuBr}(\mathrm{dNbpy})_{2}\right)$. However, oxygen dissolved in aqueous media can readily oxidize the transition metal in a catalyst complex, resulting in a loss of ATRP activator and reduction of the reaction rate. Thus, experimental procedures requires care in catalyst handling and in the removal of oxygen from the reaction mixture.

Reverse ATRP is a convenient method for reducing the oxidation problem encountered in normal ATRP. In reverse ATRP, transition metal complex in the higher oxidation state (i.e. $\mathrm{Cu}$ (II) complex) are added to the reaction and results in the generation of radicals through thermal decomposition of conventional free radical initiator. The initiator radicals react with the catalyst complex in higher oxidation state to form alkyl halide initiator radical and a catalyst complex in a lower oxidation state. After initial reduction step, the reaction proceeds as a normal ATRP. One advantage of the reverse initiation technique is that the components of the initial system are less sensitive to air. Consequently, reverse ATRP technique can be feasible in industrial processes. However, the main drawback for this method is the relatively high catalyst loading and the limited functionality of the polymer chains.

In contrast to reverse ATRP, another technique named simultaneous reverse and normal initiation (SR\&NI) utilizes a dual initiation system. The dual initiation system is comprised of a conventional free radical initiator, a higher oxidation state metal catalyst complex and an initiator with a transferrable atom or alkyl halide. The initiating radicals generated by the primary initiator radicals are deactivated by $\mathrm{Cu}$ (II) $\mathrm{Br} / \mathrm{L}$ complex to form $\mathrm{Cu}(\mathrm{I}) \mathrm{Br} / \mathrm{L}$. The $\mathrm{Cu}(\mathrm{I}) \mathrm{Br} / \mathrm{L}$ can then activate the alkyl halide initiator and concurrently mediate normal ATRP. However, the use of conventional 
initiator radical introduces new free radicals leading to the production of homopolymer chains. As a result, pure block copolymers are unlikely to obtain in SR\&NI ATRP process.

\section{A.3 Activator Generated by Electron Transfer (AGET) ATRP}

In Activator Generated by Electron Transfer (AGET) ATRP, chemical reducing agents are utilized to quickly reduce the higher oxidation state of the catalyst complex. The reducing agent reacts with the higher oxidation state transition metal complex, but the oxidized products cannot initiate new chains. Therefore, pure linear block copolymers and star block copolymers can be synthesized without the presence of any homopolymers.

Min reported the first miniemulsion AGET ATRP. AGET ATRP can be carried out in limited amount of air presence in miniemulsion and bulk polymerization. In addition, AGET ATRP employed variety of reducing agents, including $\mathrm{Sn}^{\mathrm{II}}$ compounds (i.e tin (II) 2-ethyl-hexanoate), or ascorbic acid. An excess of reducing agent was utilized to consume the oxygen present in the system. AGET ATRP has significant advantages because most reagents are stable in the presence of air and no homopolymers are produced during block copolymerization.

\section{A.4 Initiators for Continuous Activator Regeneration (ICAR) ATRP}

In Initiators for Continuous Activator Regeneration (ICAR) ATRP, free radicals slowly regenerates a very low concentration of $\mathrm{Cu}(\mathrm{I})$ activator complex. The ICAR ATRP requires a slow decomposition of a thermal radical initiator such as AIBN. Furthermore, a fraction of initiator should remain at the end of the reaction since the AIBN is completely depleted the reaction stops (Matyjaszewski and Tsarevsky, 2014). Thus, the kinetics of ICAR ATRP resembles a convention radical polymerization and depends on the rate of AIBN decomposition (Zhong and Matyjaszewski, 2011).

In ICAR ATRP, like SR\&NI ATRP, small fraction of chains (typically 5-15mol \%), that are generated, originate from the added thermal initiator. As a result, ICAR ATRP is not an appropriate synthesis procedure if pure block copolymers are desired product. If the temperature is not controlled precisely, the radical initiator may quickly decompose and lead to uncontrolled fast and exothermic polymerization. 


\section{A.5 Activator Regenerated by Electron Transfer (ARGET) ATRP}

Activator Regenerated by Electron Transfer (ARGET) ATRP is not just another way to initiate an ATRP but also the "green" way to run CRP (Matyjaszewski and Tsarevsky, 2014). ARGET ATRP procedure used much lower concentration of catalyst in a system that is applicable for industrial scale-up and produces pure $\alpha$-functional products.

The challenges encounter with ICAR ATRP such as: (i) the formation of polymers originating from the radical sources or (ii) possibility of runaway reaction, could be resolved with dosing of non-radical forming reducing agents used in ARGET ATRP (Matyjaszewski and Tsarevsky, 2014). Typically, reducing agents include $\mathrm{Sn}^{\mathrm{II}} \mathrm{R}_{2}$ compounds, glucose, ascorbic acid, $\mathrm{Ag}$ or hydrazine. Additionally, certain monomers and ligands can be used as internal reducing agents. Low dosing of the reducing agent can improve the rate of reduction and thus the rate of polymerization (Matyjaszewski and Tsarevsky, 2014). Selection of a suitable reducing agent is of great importance, since it needs to ensure that both the reducing agent and its oxidizing product, do not interfere with the reagents added for the desired ATRP. Various side reactions can lead to poor control such as (i) complexation or reaction with the ligand, monomer or polymer, (ii) complexation of reducing agent with the metal centre of the catalyst complex, (iii) protonation of the ligand by acidic oxidation products and (iv) nucleophilic substitution or elimination of halide chain ends (Woodruff et al., 2012). 


\section{REFERENCE}

Aldabbagh, F., Zetterlund, P. B., \& Okubo, M. (2008). Nitroxide-mediated precipitation polymerization of styrene in supercritical carbon dioxide: Effects of monomer loading and nitroxide partitioning on control. European Polymer Journal, 44(12), 4037-4046.

Antonietti, M., Basten, R., \& Lohmann, S. (1995). Polymerization in microemulsions - a new approach to ultrafine, highly functionalized polymer dispersions. Macromolecular Chemistry and Physics, 196(2), 441-466.

Apostolovic, B., Quattrini, F., Butté, A., Storti, G. \& Morbidelli, M., 2006. Ab initio Emulsion Polymerization by RAFT (Reversible Addition-Fragmentation Chain Transfer) through the Addition of Cyclodextrins. Helvetica Chimica Acta, 89(8), 1641-1659.

Arshady, R. (1992). Suspension, emulsion, and dispersion polymerization: A methodological survey. Colloid \& Polymer Science, 270(8), 717-732.

Asua, J. M. (2002). Miniemulsion Polymerization. Progress in Polymer Science, 27 (7), 12831346.

Awad, M., Dhib, R. \& Duever, T., 2020. Influence of HMTA ligand in MMA AGET ATRP emulsion polymerization. Journal of Applied Polymer Science, 49128.

Ayres, N. (2011). Atom Transfer Radical Polymerization: A Robust and Versatile Route for Polymer Synthesis. Polymer Reviews, 51(2), 138-162.

Barner-Kowollik, C., Vana, P., \& Davis, T. P., (2002). The Kinetics of Free-Radical Polymerization. Handbook of Radical Polymerization. Hobojen, N.J.: Wiley-Interscience.

Blackley, D. (1997). Emulsion polymerization: A mechanistic approach. Polymer, 38(10), 25772578.

Bergenudd, H. (2011). Understanding the mechanism behind atom transfer radical polymerization exploring the limit of control. Chemical Science and Engineering, KTH Royal Institute of Technology, Stockholm. 
Borman, S. (2006). Polymers with Safe Amounts of Copper. Chemical \& Engineering News, 84(44), 40-41.

Braunecker, W. A., \& Matyjaszewski, K. (2007). Controlled/living radical polymerization: Features, developments, and perspectives. Progress in Polymer Science, 32(1), 93-146.

Candau, F. (1990). Polymerization in Inverse Emulsions and Microemulsions. An Introduction to Polymer Colloids, Springer Netherlands, 73-96.

Chan, N., Cunningham, M. F., \& Hutchinson, R. A. (2013). Copper-mediated controlled radical polymerization in continuous flow processes: Synergy between polymer reaction engineering and innovative chemistry. Journal of Polymer Science Part A: Polymer Chemistry, 51(15), 3081-3096.

Chan-Seng, D., \& Georges, M. K. (2006). Living radical emulsion polymerization using the nanoprecipitation technique: An extension to atom transfer radical polymerization. Journal of Polymer Science Part A: Polymer Chemistry, 44(13), 4027-4038.

Chauvin, F., Dufils, P.-E., Gigmes, D., Guillaneuf, Y., Marque, S. R. A., Tordo, P., \& Bertin, D. (2006). Nitroxide-Mediated Polymerization: The Pivotal Role of the $\mathrm{k}_{\mathrm{d}}$ Value of the Initiating Alkoxyamine and the Importance of the Experimental Conditions. Macromolecules, 39(16),

Chern, C. (2006). Emulsion polymerization mechanisms and kinetics. Progress in Polymer Science, 31(5), 443-486.

Coleman, B. \& Fox, T., (1963). Multistate Mechanism for Homogeneous Ionic Polymerization. I. The Diastereosequence Distribution. The Journal of Chemical Physics, 38(5), 1065-1075.

Destarac, M. (2010). Controlled Radical Polymerization: Industrial Stakes, Obstacles and Achievements. Macromolecular Reaction Engineering, 4(3-4), 165-179.

Dhib, R., Gao, J. \& Penlidis, A., (2000). Simulation of Free Radical Bulk/Solution Homopolymerization Using Mono- and Bi-functional Initiators. Polymer Reaction Engineering, 8(4), 299-464. 
El-Aasser, M. S., \& Lovell, P. A. (1998). Emulsion polymerization and emulsion polymers. Chichester: John Wiley and Sons.

Enright, T. E., Cunningham, M. F., \& Keoshkerian, B. (2005). Nitroxide-Mediated Polymerization of Styrene in a Continuous Tubular Reactor. Macromolecular Rapid Communications, 26(4), 221-225.

Roudsari S.F (2015), Experimental and CFD investigation of the mixing of MMA emulsion polymerization in a stirred tank reactor.

Fischer, H. (1999). The persistent radical effect in controlled radical polymerizations. Journal of Polymer Science Part A: Polymer Chemistry, 37(13), 1885-1901.

Fischer, H. \& Fukuda, T. (2001). The Persistent Radical Effect: A Principle for Selective Radical Reactions and Living Radical Polymerizations. Chemical Reviews, 101(12), 3581-3610.

Georges, M. K., Veregin, R. P. N., Kazmaier, P. M., \& Hamer, G. K. (1993). Narrow molecular weight resins by a free-radical polymerization process. Macromolecules, 26(11), 29872988.

Gilbert, R. G. (1995). Emulsion polymerization: a mechanistic approach. London: Academic.

Goto, A., \& Fukuda, T. (1997). Effects of Radical Initiator on Polymerization Rate and $\begin{array}{llll}\text { Polydispersity in Nitroxide-Controlled } & \text { Free }\end{array}$ Polymerization. Macromolecules, 30(15), 4272-4277

Goto, A., \& Fukuda, T. (1999). Kinetic Study on Nitroxide-Mediated Free Radical Polymerization oftert-Butyl Acrylate. Macromolecules, 32(3), 618-623.

Haddleton, D. M., Kukulj, D., Duncalf, D. J., Heming, A. M., \& Shooter, A. J. (1998). LowTemperature Living "Radical” Polymerization (Atom Transfer Polymerization) of Methyl Methacrylate Mediated by Copper(I)N-Alkyl-2-Pyridylmethanimine Complexes. Macromolecules, 31(16), 5201-5205.

Harkins, W. D. (1947). General theory of mechanism of emulsion polymerization. II. Journal of Polymer Science, 5(2), 217-251. 
Hawker, C. J. Nitroxide-Mediated Living Radical Polymerizations. Handbook of Radical Polymerization, 463-521.

Ibrahim, K., Yliheikkilä, K., Abu-Surrah, A., Löfgren, B., Lappalainen, K., Leskelä, M., Repo, T. \& Seppälä, J., (2004). Polymerization of methyl methacrylate in the presence of iron(II) complex with tetradentate nitrogen ligands under conditions of atom transfer radical polymerization. European Polymer Journal, 40(6), 1095-1104.

Inoue, Y., \& Matyjaszewski, K. (2004). New Amine-Based Tripodal Copper Catalysts for Atom Transfer Radical Polymerization. Macromolecules, 37(11), 4014-4021.

Isse, A., Lorandi, F.\& Gennaro, A., (2019). Electrochemical approaches for better understanding of atom transfer radical polymerization. Current Opinion in Electrochemistry, 15, 50-57.

Kreutzer, J., (2018). Atom-transfer radical polymerization: New method breathes life into ATRP. Nature Reviews Chemistry, 2(2).

Krys, P., Ribelli, T. G., Matyjaszewski, K., \& Gennaro, A. (2016). Relation between Overall Rate of ATRP and Rates of Activation of Dormant Species. Macromolecules, 49(7), 24672476.

Krys, P., Matyjaszewski, K., (2017) Kinetics of atom transfer radical polymerization; European Polymer Journal ,89, 482-523

Landfester, K. (2000). Recent developments in miniemulsions - formation and stability mechanisms. Macromolecular Symposia, 150(1), 171-178.

Landfester, K. (2001). Polyreactions in Miniemulsions. Macromolecular Rapid Communications, 22(12), 896-936.

Landfester, K. (2003). Miniemulsions for Nanoparticle Synthesis. Topics in Current Chemistry Colloid Chemistry II, 75-123.

Li, L., (2007) Fundamental Kinetic and Mechanistic Studies of the Stable Free Radical Polymerization (SFRP) Process. 
Li, M., \& Matyjaszewski, K. (2003). Further progress in atom transfer radical polymerizations conducted in a waterborne system. Journal of Polymer Science Part A: Polymer Chemistry, 41(22), 3606-3614.

Li, W., \& Matyjaszewski, K. (2011). Cationic Surface-Active Monomers as Reactive Surfactants for AGET Emulsion ATRP ofn-Butyl Methacrylate. Macromolecules, 44(14), 5578-5585.

Lorandi, F., Wang, Y., Fantin, M. \& Matyjaszewski, K., (2018). Ab Initio Emulsion AtomTransfer Radical Polymerization. Angewandte Chemie, 130(27), 8402-8406.

Louie, B. M., Carratt, G. M., \& Soong, D. S. (1985). Modeling the free radical solution and bulk polymerization of methyl methacrylate. Journal of Applied Polymer Science, 30(10), $3985-4012$.

Massicotte, E., 2016. Modelling and experimental investigation of ARGET/AGET atom transfer radical polymerization systems.

Mastan, E., Zhou, D. \& Zhu, S., (2014). Development of Molecular Weight Distribution in ATRP with Radical Termination. Macromolecular Theory and Simulations, 23(3), 227-240.

Mastan, E. \& Zhu, S., (2015). A Molecular Weight Distribution Polydispersity Equation for the ATRP System: Quantifying the Effect of Radical Termination. Macromolecules, 48(18), 6440-6449.

Matyjaszewski, K., \& Xia (n.d), J. Fundamentals of Atom Transfer Radical Polymerization. Handbook of Radical Polymerization, 523-628.

Matyjaszewski, K., Coca, S., Gaynor, S. G., Wei, M., \& Woodworth, B. E. (1997). Zerovalent Metals in Controlled/ "Living" Radical Polymerization. Macromolecules, 30(23), 73487350.

Matyjaszewski, K. (1997). Mechanistic and Synthetic Aspects of Atom Transfer Radical Polymerization. Journal of Macromolecular Science, Part A, 34(10), 1785-1801.

Matyjaszewski, K. (Ed.). (2003). Advances in Controlled/Living Radical Polymerization (Vol. 854). Washington, DC: American Chemical Society. 
Matyjaszewski, K. General Concepts and History of Living Radical Polymerization. Handbook of Radical Polymerization, 361-406.

Matyjaszewski, K. (2012). Atom Transfer Radical Polymerization: From Mechanisms to Applications. Israel Journal of Chemistry, 52(3-4), 206-220.

Matyjaszewski, K. (2014). From cationic ring-opening polymerization to atom transfer radical polymerization. Polimery, 59(01), 24-37.

Min, K., \& Matyjaszewski, K. (2005). Atom Transfer Radical Polymerization in Microemulsion. Macromolecules, 38(20), 8131-8134.

Min, K., Jakubowski, W., \& Matyjaszewski, K. (2006). AGET ATRP in the Presence of Air in Miniemulsion and in Bulk. Macromolecular Rapid Communications, 27(8), 594-598.

Min, K., Gao, H., \& Matyjaszewski, K. (2006). Development of an ab Initio Emulsion Atom Transfer Radical Polymerization: From Microemulsion to Emulsion. Journal of the American Chemical Society, 128(32), 10521-10526.

Moad, G., Rizzardo, E. and Solomon, D., (1982). A product study of the nitroxide inhibited thermal polymerization of styrene. Polymer Bulletin, 6(11-12).

Moad, G. \& Rizzardo, E., (1995). Alkoxyamine-Initiated Living Radical Polymerization: Factors Affecting Alkoxyamine Homolysis Rates. Macromolecules, 28(26), 8722-8728.

Moad G, Anderson AG, Ercole F, Johnson HJ, Krstina J, Moad CL, Rizzardo E, Spurling TH, \& Thang SH, (1998). Controlled-growth free radical polymerization of methacrylate esters: reversible chain transfer versus reversible termination. ACS Symposium Series, 685, 33260

Monteiro, M. \& Cunningham, M., (2012). Polymer Nanoparticles via Living Radical Polymerization in Aqueous Dispersions. Design and Applications. Macromolecules, 45(12), 4939-4957

Nabifar, A., (2007) Investigations of kinetic aspects in nitroxide-mediated radical polymerization of styrene. Ann Arbor: University of Waterloo (Canada). 
Nicolas, J., Guillaneuf, Y., Lefay, C., Bertin, D., Gigmes, D. \& Charleux, B., (2013) NitroxideMediated Polymerization. Progress in Polymer Science 38 (1,), 63-235

Nicolas, J., \& Guillaneuf, Y. (2015). Living Radical Polymerization: Nitroxide-Mediated Polymerization. Encyclopedia of Polymeric Nanomaterials, 1133-1148.

Odian, G., (2004)"Radical Chain Polymerization". In Principles of Polymerization; John Wiley \&Sons, Inc: Hobokon, 198.

Oh, J. K. (2008). Recent advances in controlled/living radical polymerization in emulsion and dispersion. Journal of Polymer Science Part A: Polymer Chemistry, 46(21), 6983-7001.

Peng, H., Cheng, S., Feng, L., \& Fan, Z. (2003). Atom transfer radical polymerization ofn-butyl methacrylate in an aqueous dispersed system. Journal of Applied Polymer Science, 89(12), $3175-3179$.

Peng, H., Cheng, S., \& Feng, L. (2004). Reverse atom transfers radical polymerization ofn-butyl methacrylate in an aqueous dispersed system. Polymer International, 53(7), 828-832.

Penlidis, A. (1986). Polymer reactor design, optimization and control in latex production technology.

Percec, V. \& Lligadas, G., (2007). Synthesis of perfectly bifunctional polyacrylates by singleelectron-transfer living radical polymerization. Journal of Polymer Science Part A: Polymer Chemistry, 45(20), 4684-4695.

Pintauer, T., 2016. Towards the development of highly active copper catalysts for atom transfer radical addition (ATRA) and polymerization (ATRP). Chemical Papers, 70(1).

Qiu, J., Gaynor, S. G., \& Matyjaszewski, K. (1999). Emulsion Polymerization ofn-Butyl Methacrylate by Reverse Atom Transfer Radical Polymerization. Macromolecules, 32(9), $2872-2875$.

Qu, J.-B., Huan, G.-S., Chen, Y.-L., Shao, H.-H., \& Wang, L. (2013). Exploration of the Bulk Atom Transfer Radical Polymerization of Styrene. Advances in Polymer Technology, 32(4). 
Ribelli, T., Lorandi, F., Fantin, M. \& Matyjaszewski, K., (2018). Atom Transfer Radical Polymerization: Billion Times More Active Catalysts and New Initiation Systems. Macromolecular Rapid Communications, 40(1), 1800616.

Roa-Luna, M., Nabifar, A., Díaz-Barber, M. P., Mcmanus, N. T., Vivaldo-Lima, E., Lona, L. M., \& Penlidis, A. (2007). Another Perspective on the Nitroxide Mediated Radical Polymerization (NMRP) of Styrene Using 2,2,6,6-Tetramethyl-1-piperidinyloxy (TEMPO) and Dibenzoyl Peroxide (BPO). Journal of Macromolecular Science, Part A, 44(3), 337-349.

Save, M., Guillaneuf, Y., \& Gilbert, R. G. (2007). Controlled Radical Polymerization in Aqueous Dispersed Media. ChemInform, 38(8).

Sawamoto, M., Nishikawa, T., Ando, T., \& Kamigaito, M.(1995). Evidence for Living Radical Polymerization of Methyl Methacrylate with Ruthenium Complex: Effects of Protic and Radical Compounds and Reinitiation from the Recovered Polymers1. Macromolecules, 30(8), 2244-2248.

Schulz, W., (1980). Modern size-exclusion liquid chromatography: Practice of gel permeation and gel filtration chromatography. Journal of Chromatography A, 195(2), 306.

Shen, Y., Tang, H., \& Ding, S. (2004). Catalyst separation in atom transfer radical polymerization. Progress in Polymer Science, 29(10), 1053-1078.

Shipp, D.A., Matyjaszewski, K., Wang, J.-L., \& Grimaud, T. (1998). Controlled/ “Living” Atom Transfer Radical Polymerization of Methyl Methacrylate Using Various Initiation Systems. Macromolecules, 31(5), 1527-1534.

Simms, R. W., \& Cunningham, M. F. (2007). High Molecular Weight Poly (butyl methacrylate) by Reverse Atom Transfer Radical Polymerization in Miniemulsion Initiated by a Redox System. Macromolecules, 40(4), 860-866.

Striegel, A., Yau, W., Kirkland, J. \& Bly, D., (2009). Modern Size-Exclusion Liquid Chromatography.

Surmacz, K. \& Chmielarz, P., (2020). Low Ppm Atom Transfer Radical Polymerization in (Mini)Emulsion Systems. Materials, 13(7), 1717. 
Szwarc, M. (1956) “Living” polymers. Nature, 176:1168-9.

Tang, W., Kwak, Y., Braunecker, W., Tsarevsky, N. V., Coote, M. L., \& Matyjaszewski, K. (2008). Understanding Atom Transfer Radical Polymerization: Effect of Ligand and Initiator Structures on the Equilibrium Constants. Journal of the American Chemical Society, 130(32), 10702-10713.

Teodorescu, M., \& Matyjaszewski, K. (1999). Atom Transfer Radical Polymerization of (Meth)acrylamides. Macromolecules, 32(15), 4826-4831.

Tsarevsky, N. V., \& Sumerlin, B. S. (2013) Fundamentals of Controlled/Living Radical Polymerization; Royal Society of Chemistry: Cambridge

Tuinman, E., McManus, N., Roa-Luna, M., Vivaldo-Lima †, E., Lona, L. \& Penlidis, A., (2006). Controlled Free-Radical Copolymerization Kinetics of Styrene and Divinylbenzene by Bimolecular NMRP using TEMPO and Dibenzoyl Peroxide. Journal of Macromolecular Science, Part A, 43(7), 995-1011.

Upadhayay Regmi, K. (2016), Experimental investigation of emulsion AGET ATRP of MMA in a stirred tank reactor.

Upadhayay Regmi, K., Mehrvar, M. \& Dhib, R., (2017). Single- and two-step procedures of AGET emulsion ATRP of methyl methacrylate in a well-mixed batch reactor. Journal of Applied Polymer Science, 134(38), 45308.

Upadhayay Regmi, K., Mehrvar, M. \& Dhib, R., (2019). Experimental Design and Statistical Analysis of AGET ATRP of MMA in Emulsion Polymer Reactor. Macromolecular Reaction Engineering, 13(4), 1900006.

Van Camp, W., Germonpré, V., Mespouille, L., Dubois, P., Goethals, E. \& Du Prez, F., (2007). New poly(acrylic acid) containing segmented copolymer structures by combination of "click" chemistry and atom transfer radical polymerization. Reactive and Functional Polymers, 67(11), 1168-1180.

Wang, J.-S., \& Matyjaszewski, K. (1995). Controlled/"living" radical polymerization. atom transfer radical polymerization in the presence of transition-metal complexes. Journal of the American Chemical Society, 117(20), 5614-5615. 
Wei, Y., Jia, Y., Wang, W., Li, B. \& Zhu, S., (2014). Surfactant-Ligand Design for ab Initio Emulsion Atom Transfer Radical Polymerization. Macromolecules, 47(22), 7701-7706.

Woodruff, S. R., Davis, B. J., \& Tsarevsky, N. V. (2012). Selecting the Optimal Reaction Conditions for Copper-Mediated Atom Transfer Radical Polymerization at Low Catalyst Concentration. ACS Symposium Series Progress in Controlled Radical Polymerization: Mechanisms and Techniques, 99-113.

WYATT Technology (2014), DynaPro PlateReader II User’s Guide, California, USA

Yong, C. P., \& Gan, L. M. (2005). Microemulsion Polymerizations and Reactions. Polymer Particles Advances in Polymer Science, 257-298.

Zetterlund, P. B., Saka, Y., Mchale, R., Nakamura, T., Aldabbagh, F., and Okubo, M. (2006), Polymer, 47(23), 7900-7908.

Zhang, M. \& Ray, W., (2001). Modeling of "Living” Free-Radical Polymerization with RAFT Chemistry. Industrial \& Engineering Chemistry Research, 40(20), 4336-4352.

Zhong, M., \& Matyjaszewski, K. (2011). How Fast Can a CRP Be Conducted with Preserved Chain End Functionality? Macromolecules, 44(8), 2668-2677. 